\title{
Cartilage Adjacent Subchondral Bone in Ageing and Disease as a Diagnostic and Therapeutic Target
}

\author{
Dissertation for the award of the degree \\ “Doctor of Philosophy” (Ph.D.) \\ within the doctoral program in \\ "Medical Human Sciences"
}

Submitted by:

Shahed Taheri

Born 31.05.1987 in Gachsaran, Iran

to

the Department of Trauma Surgery, Orthopedics and Plastic Surgery

University Medical Center Göttingen

Göttingen, January 2021 


\section{PhD Thesis Committee:}

Supervisor

Second Thesis Committee Member

Third Thesis Committee Member

Examination Committee
Prof. Dr. med. Arndt F Schilling

Head of Research and Development at

Department of Trauma Surgery, Orthopedics and Plastic Surgery, University Medical Center Göttingen (UMG)

Prof. Dr. med. Frauke Alves

Group leader "Translational Molecular Imaging in Oncology "Max Planck Institute for Experimental Medicine.

Prof. Dr. rer. nat. Carolin Wichmann

Group Leader "InnerEarLab", Group Center for Biostructural Imaging of Neurodegeneration (BIN) Institute for Auditory Neuroscience \& InnerEarLab, University Medical Center Göttingen

PD Dr. sc. hum. Christian Dullin

Institute for Diagnostic and Interventional Radiology, University Medical Center Göttingen

Prof. Dr. rer. nat. Uwe Kornak

Management of the genetic counseling center of the Institute for Human Genetics, University Medical Center Göttingen

Prof. Dr. med. Heide Siggelkow

Head of the Working Groups Molecular Endocrinology and Osteology, UMG 


\section{Declaration}

Herewith I declare that this thesis entitled "Cartilage adjacent subchondral bone in ageing and disease as a diagnostic and therapeutic target" is my original work, is prepared independently, and with no other sources and aids than quoted.

Göttingen, 06 January 2021

Shahed Taheri 


\section{Table of contents}

List of abbreviations ..................................................................................................

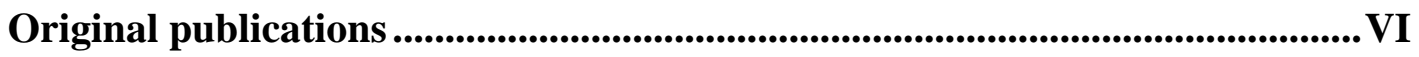

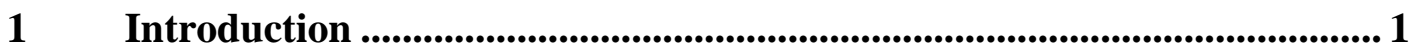

1.1 Basic anatomical features and functions of subchondral bone ................................ 2

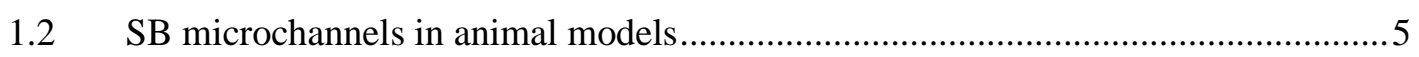

1.3 Microarchitecture of SB in healthy human knee joint .......................................... 6

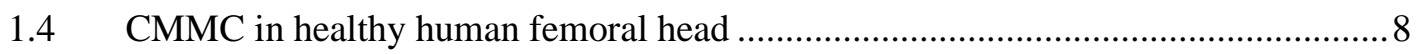

1.5 Connective microfeatures in other human joints .................................................. 10

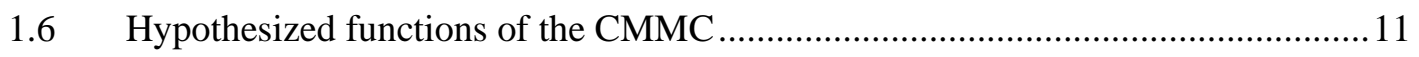

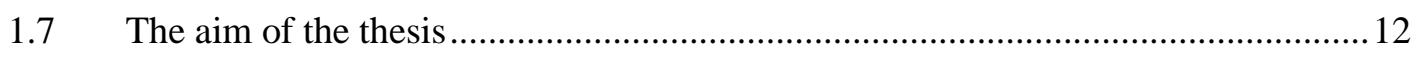

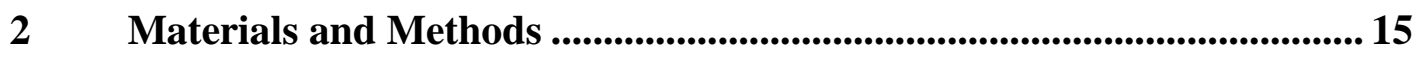

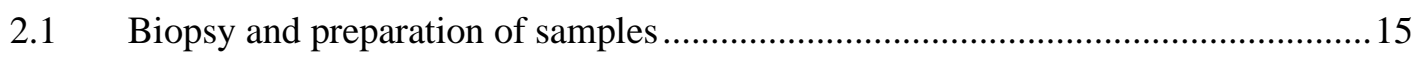

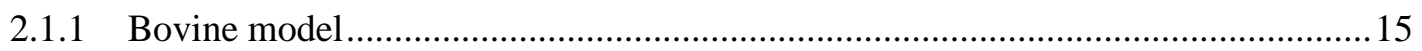

2.1.2 Healthy and early osteoarthritic human femoral heads............................................ 16

2.1.3 Murine destabilization-induced osteoarthritis model...........................................23

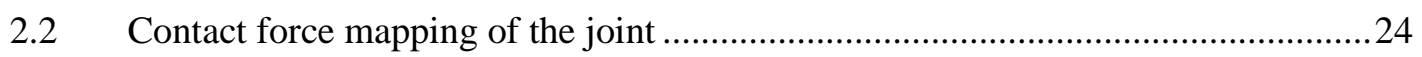

2.3 Micro-computed tomography (micro-CT) …......................................................25

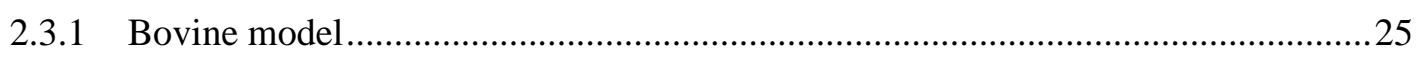

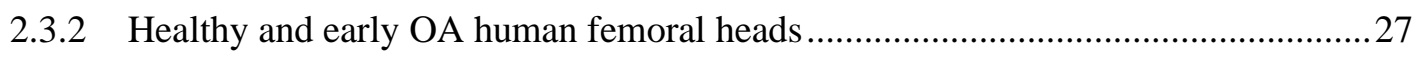

2.3.3 Murine destabilization-induced osteoarthritis model................................................30

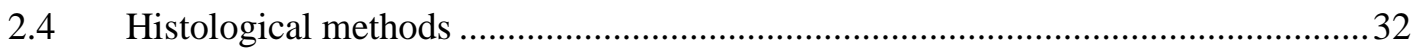

2.4.1 Mason-Goldner staining of the osteochondral junction in the bovine model ............32

2.4.2 Staining of the subchondral bone in human femoral heads ...................................... 33

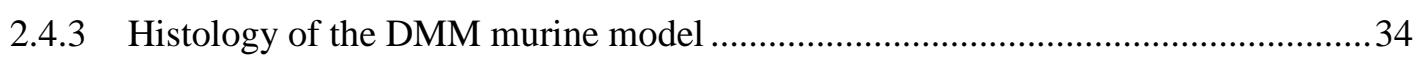

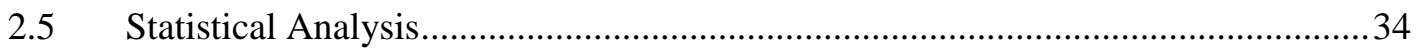

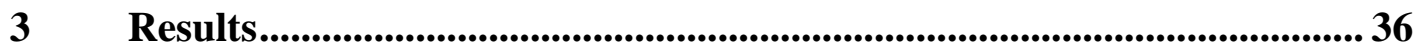

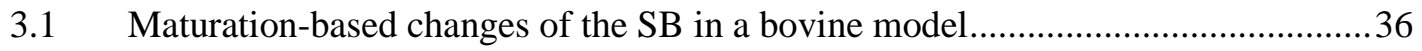

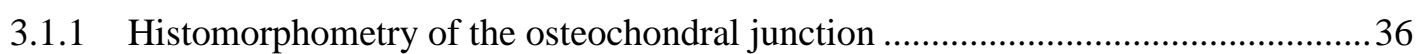

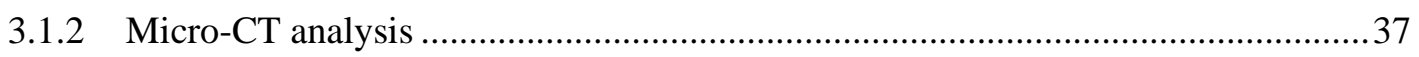

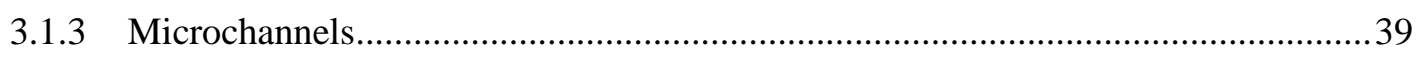

3.2 Mapping of the SB microchannels in healthy human femurs ................................. 41

3.2.1 The superolateral area of the femoral head is the load-bearing region .....................41

3.2.2 The thickest area of cartilage and subchondral bone coincide..................................42

3.2.3 Cartilage is connected to the marrow cavity via a complex microchannel network 42

3.2.4 Microchannels often appear in coronal histological sections as remote pores .........44 
3.2.5 The LBR is characterized by copious small microchannels, while large, infrequent, and elongated microchannels typify the SB microstructure in the peripheral rim of

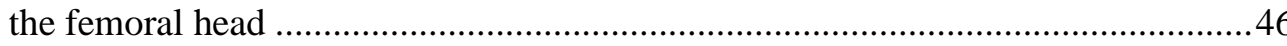

3.2.6 Cartilage thickness is positively associated with the SB thickness, CMMC number,

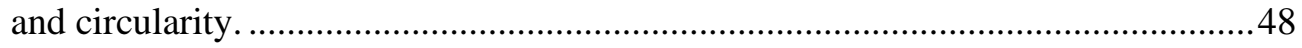

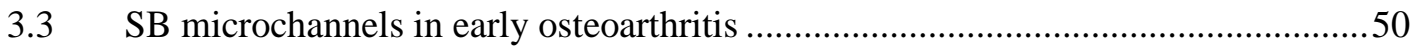

3.3.1 Partial thickness defect and early cartilage fibrillation occurs prevalently at the

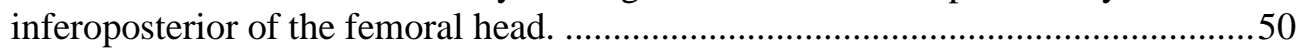

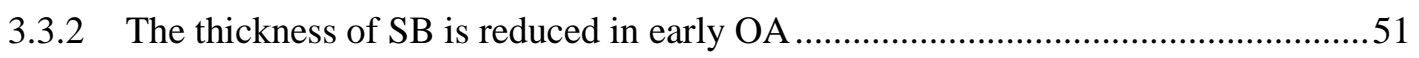

3.3.3 The CMMC maintain their location-dependent characteristic in early OA.............52

3.3.4 The local density of CMMC does not change in early OA but their size is increased 53

3.4 Early and late subchondral bone changes in a traumatic OA model ......................56

3.4.1 DMM surgery induces progressive cartilage degradation ........................................56

3.4.2 Traumatic OA induction leads to thickening of the calcified cartilage ....................56

3.4.3 OA induction leads to irregular formation of osteophytes at the outermost rim of the

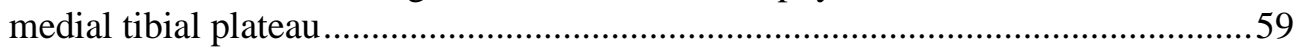

3.4.4 Medial subchondral bone sclerosis progresses during the course of traumatic OA 60

3.4.5 Traumatic OA induces meniscal ectopic bone formation .........................................61

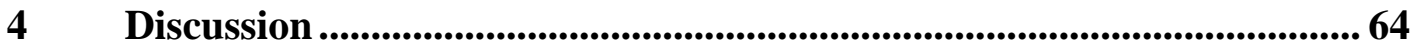

4.1 Maturation-based changes of the subchondral bone ............................................64

4.2 Mapping of the SB microchannels in healthy human femurs .................................69

4.2.1 Extended possible functions of the CMMC based on the new results......................73

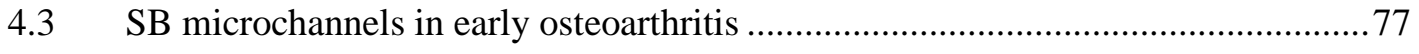

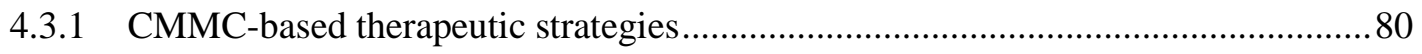

4.4 Early and late subchondral bone changes in a traumatic OA model ........................83

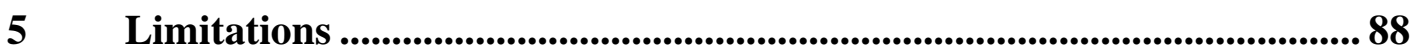

5.1 Maturation-based changes of the subchondral bone ............................................ 88

5.2 Mapping of the SB microchannels in healthy and early OA human femurs ............8 88

5.3 Early and late subchondral bone changes in a traumatic OA model .......................89

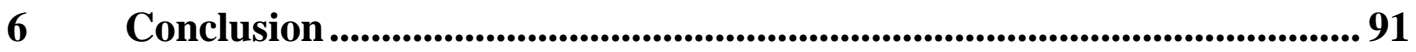

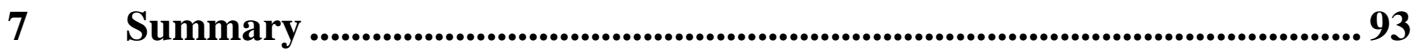

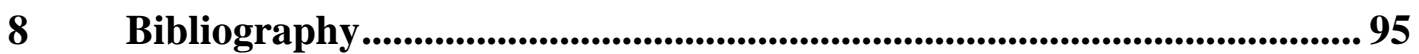

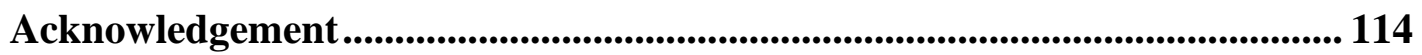




\section{List of figures}

Figure 1. Different features of articular cartilage and subchondral bone.............................

Figure 2. A schematic model for the SB microchannels in human tibial plateau. .................. 8

Figure 3. Preparation of cartilage-bone biopsies from the bovine bone. ............................ 15

Figure 4. The PCR-based sex determination of the healthy human femurs.......................... 18

Figure 5. Schematic illustration of the cortical bone's microstructure in various age-groups.

Figure 6. An exemplary microscopic image of the cortical bone's microstructure. ............21

Figure 7. The system for assigning measuring points on the femoral head.........................22

Figure 8. Workflow for 3D model generation of femurs by photogrammetry.....................23

Figure 9. Volume of interests for measurement of different SB layers in calf. ....................26

Figure 10. Measurement of bone mineralization in the bovine model................................22

Figure 11. The scout view of a $2.00 \mathrm{~mm}$ cartilage-bone biopsy.......................................28

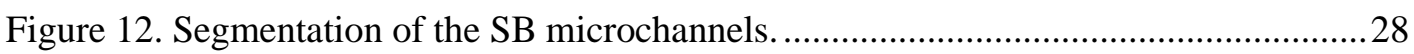

Figure 13. Masson-Goldner staining of the osteochondral junction. ...................................36

Figure 14. The image reconstruction of SB and subarticular region in bovine.....................37

Figure 15. Profiles for the bone mineralization changes in the bovine model.....................39

Figure 16. The 3D representation of the SB microchannels in the bovine model. ..............40

Figure 17. Contact force mapping of the femoral head................................................. 41

Figure 18. The changes of the AC and SB thicknesses in different loading areas of the

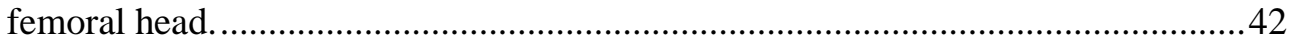

Figure 19. 3D representation of the SB microchannel network in healthy human femoral heads.

Figure 20. 3D imaging-histology juxtaposition of the osteochondral junction....................44

Figure 21. Depiction of the osteochondral junction by histology / immunohistochemistry.45

Figure 22. Quantitative analysis of the CMMC in the healthy human femoral heads........ 47

Figure 23. Region-based morphological changes of the SB microchannels in the femoral head.

Figure 24. The distribution of early AC fibrillation and partial thickness defect on the femoral head.

Figure 25. The early OA characteristics of the osteochondral unit in human femoral head.

Figure 26. 3D representation of the SB microchannel network in early OA femoral heads.

Figure 27. Quantitative analysis of the CMMC in the early OA human femoral heads......55

Figure 28. Safranin-O staining of the WT, Tac1-/-, and $\alpha$ CGRP-/- knee joints................57

Figure 29. Calcified cartilage heat map of the mice tibial plateau 2 weeks after OA induction.

Figure 30. Calcified cartilage heat map of the mice tibial plateau 8 weeks after DMM

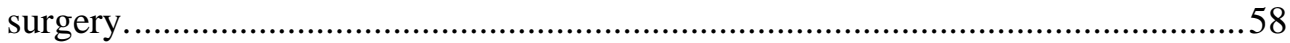

Figure 31. Diagrams of the mean CC thickness in the medial and lateral tibia plateaus.....59

Figure 32. Osteophyte formation after DMM surgery in wildtype and neuropeptideknockout mice.

Figure 33. The influence of traumatic OA induction and neuropeptide loss on bone sclerosis

Figure 34. Meniscal ectopic bone formation after DMM surgery. 
Figure 35. Comparison of the BMD in the medial tibial epiphysis and the meniscal ossicles.

Figure 36. A schematic representation of the new CMMC-based therapeutic strategy for cartilage repair.

Figure 37. The revised model of the osteochondral unit..................................................92

\section{List of tables}

Table 1. Characteristics of SB perforations in animal models and the human knee joint...... 7

Table 2. Characteristics of SB perforations in the human femoral head.

Table 3. Quality characteristics of the extracted DNAs from healthy and early OA femurs

Table 4. Biological age determination of the healthy and early OA femurs.......................20

Table 5. Micro-CT data of the entire subchondral zone in the bovine model........................37

Table 6. Layer-by-layer micro-CT data of the SB in the bovine model ...............................38

Table 7. Correlation of the AC thickness with different variables of the SB microarchitecture 


\section{List of abbreviations}

2D

3D

$\mathrm{AC}$

ANOVA

BMD

BV

$\mathrm{BV} / \mathrm{TV}$

$\mathrm{CC}$

Circ.

CMMC

Conn.D.

DA

DMM

Feret

$\mathrm{H} \& \mathrm{E}$

$\mathrm{KO}$

LBR

$\mathrm{MHH}$

Micro-CT

MinFeret

MMA

$\mathrm{NIH}$

NLBR

$\mathrm{OA}$

PCR

PR

ROI

SB

SBP

SEM

SP

SRG

Tac1-/-

Tb.N.

Tb.Sp.

Tb.Th.

VOI

vWF

WT

$\alpha C G R P$ two-dimensional

three-dimensional

articular cartilage

analysis of variance

Bone mineral density

bone volume

bone volume fraction

calcified cartilage

circularity index

cartilage-bone marrow microchannel connectors

connectivity density

degree of anisotropy

destabilization of the medial meniscus

maximum caliper dimeter

hematoxylin and eosin

knockout

load-bearing region

Medizinische Hochschule Hannover

microcomputed tomography

minimum caliper dimeter

methyl methacrylate

National Institutes of Health

non-load-bearing region

osteoarthritis

polymerase chain reaction

peripheral rim

region of interest

subchondral bone

subchondral bone plate

scanning electron microscopy

substance $\mathrm{P}$

seeded region growing

substance P-deficient

trabecular number

trabecular separation

trabecular thickness

volume of interest

von Willebrand Factor

wildtype

$\alpha$-Calcitonin Gene-Related Peptide 


\section{Original publications}

The following publications constitute the context of this work, and are described in detail throughout this dissertation

Taheri S*, Winkler T*, Schenk L*, Neuerburg C, Baumbach S, Zustin J, Lehmann W, Schilling AF (2019): Developmental Transformation and Reduction of Connective Cavities within the Subchondral Bone. Int J Mol Sci 20, 770

(* These authors contributed equally to this work and share the first authorship)

Muschter D*, Fleischhauer L*, Taheri S*, Schilling AF, Clausen-Schaumann H, Grässel S (2020): Sensory neuropeptides are required for bone and cartilage homeostasis in a murine destabilization-induced osteoarthritis model. Bone $\underline{133}, 115181$

(* These authors contributed equally to this work and share the first authorship)

Taheri S, Yoshida T, Böker KO, Foerster RH, Jochim L, Flux AL, Grosskopf B, Lehmann W, Schilling AF (2020): The contact force of the human femoral head correlates with the microchannel architecture inside the subchondral bone. Calcified Tissue International (under review)

Taheri S, Schilling AF: Microchannels in subchondral bone and membranes comprising same for the treatment of osteoarthritis. USPTO No.16/282,444 (iled)

Taheri S, Böker KO, Lehmann W, Schilling AF (2020): Knorpel-Knochenmark-MikroKonnektoren im subchondralen Knochen. Osteologie; 29: 1-8; DOI: 10.1055/a-12066663 


\section{Introduction}

Osteoarthritis (OA) is a major cause of pain and disability among older population, and the most common joint disease in the world (Onishi et al. 2012; Li et al. 2013). This exceedingly prevalent disorder and the resulting mobility restrictions have profound impacts on individuals as well as the society. OA not only induces chronic pain and reduces the quality of life, but may impose a distinct emotional dimension to the functional impairment as well. OA patients are afflicted with joint stiffness and pain, which is complicated to alleviate due to the participation of multitude inflammatory and mechanical nociceptive conduits (Onishi et al. 2012; Miller et al. 2015; Eitner et al. 2017). It is estimated that $10-12 \%$ of adults suffer from symptomatic OA (Dunlop et al. 2001; Hunter et al. 2014), with knee OA patients alone at a greater risk of mobility disability (i.e. requiring support for walking or climbing stairs) compared to any other medical disorder in people 65 years of age and older (Guccione et al. 1994). Since signs of social trends such as population aging, ubiquity of obesity, and joint injury are rising, it is approximated that over the next 15 years, the number of people affected by OA will rise by around $50 \%$, which calls for immediate attention to minimize the burdens of this disease (Hootman and Helmick 2006; Perruccio et al. 2006; Hunter 2011).

Although originally labeled as a "wear and tear" process mainly characterized by articular cartilage (AC) degradation, $\mathrm{OA}$ is now considered to be a progressive joint failure caused by abnormal remodelling, and involving all periarticular tissues such as subchondral bone, synovium, cancellous bone, as well as menisci, ligaments, myofascial structures, muscles and neural tissues (Grynpas et al. 1991; Loeser et al. 2012; Onishi et al. 2012). In particular, there is growing evidence that subchondral bone (SB) plays a crucial role in the initiation and progression of OA (Castañeda et al. 2012; Li et al. 2013). SB deterioration has shown to be generally associated with AC defects, while SB sclerosis is commonly regraded as a hallmark feature of OA (Madry et al. 2010; Burr and Gallant 2012; Henrotin et al. 2012). Likewise, it is reported that during OA progression, SB is hypomineralized and may develop pathological changes such as microdamage, bone cysts, and bone marrow lesions (Bettica et al. 2002; Burr and Radin 2003; Felson et al. 2003; McErlain et al. 2012). Articular cartilage and subchondral bone are intimately interlocked to each other, creating a complex functional unit called the AC-SB interface, where the properties and function of each component can be modulated by changes in 
either tissue (Yuan et al. 2014). In the following section, basic anatomical features of the SB at the AC-SB interface are summarized, while functions of the SB are discussed.

\subsection{Basic anatomical features and functions of subchondral bone}

Subchondral bone is an extremely dynamic structure that varies in architecture by region, and is located deep to the articular cartilage (Stewart and Kawcak 2018; Donell 2019; Fell et al. 2019). The term "subchondral bone" is used in the context of this dissertation to refer to the zone that separates the AC from the medullary cavity. SB is a biphasic structure composed of two entities, which are outlined by four unique anatomical features (Figure 1): (1) The tidemark (2) the calcified cartilage (3) the cement line, and (4) the subchondral bone plate (SBP) also known as the cortical end-plate (Lyons et al. 2006; Li et al. 2013; Ren et al. 2018).

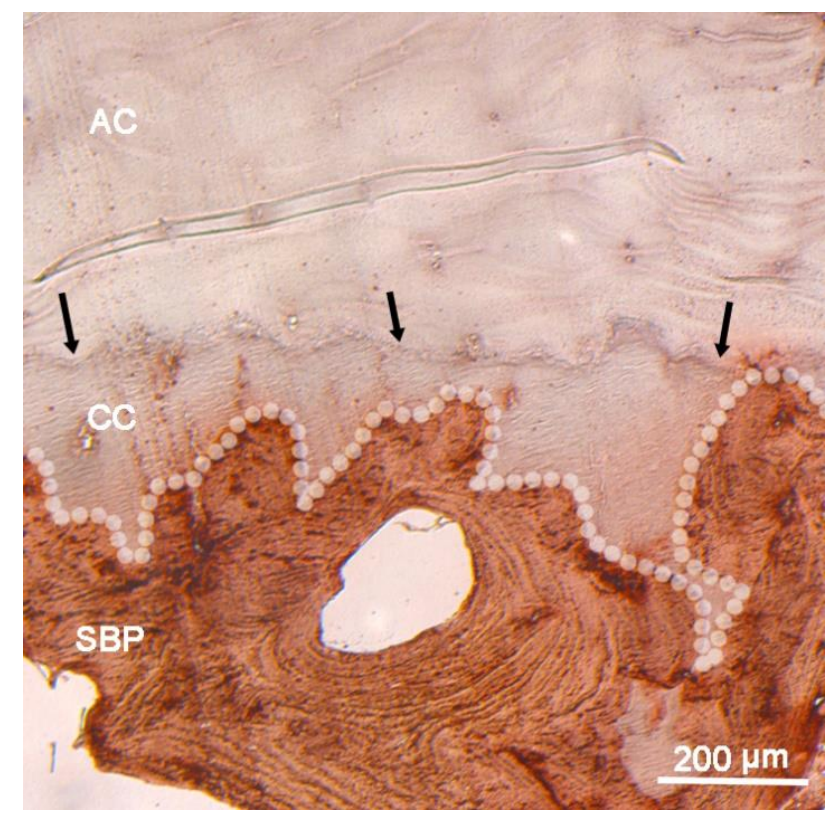

Figure 1. Different features of articular cartilage and subchondral bone. Collagen Type I Immunostaining of an exemplary bone extract from the human femoral head shows tidemark as the interface of calcified and articular cartilage layers (marked by black arrows). The white dotted line denotes the cement line, which separates $\mathrm{CC}$ from the subchondral bone plate.

The tidemark is a dynamic three-dimensional structure at the uppermost surface of the subchondral bone having a distinct trilaminate appearance, and usually seen in histological slides as a basophilic line (Lyons et al. 2005; Lyons et al. 2006; Madry et al. 2010). It denotes the bottom-most boundary line of the articular cartilage layer, directly at the subchondral bone interface. Through the tidemark, the AC is interlocked with the irregular surface of the underlying calcified cartilage; a discrete layer of mineralized 
cartilage, which consists of collagen type II, hydroxyapatite crystals, collagen type X, and glycosaminoglycan, and is radiologically seen as a denser region compared to the adjacent cartilage (Alini et al. 1992; Imhof et al. 1999; Imhof et al. 2000; Madry et al. 2010; Hoemann et al. 2012; Stewart and Kawcak 2018). As the region shifts from articular cartilage to the calcified cartilage, the rigidity of the tissue matrix changes drastically (Madry et al. 2010; Stewart and Kawcak 2018). Hence, the undulating surface of the tidemark allows for a gradual transformation of shear forces into tensile and compressive stresses (Lyons et al. 2006; Li et al. 2013). There is also a vivid margin between the calcified cartilage and the cortical end-plate, called the "cement line" (Imhof et al. 1999; Madry et al. 2010). While collagen fibrils advance through the tidemark and form a continual entity with those of the calcified cartilage, no collagen fibrils cross the cement line (Clark 1990; Clark and Huber 1990; Madry et al. 2010; Li et al. 2013). Lying distally to the $\mathrm{CC}$ is a thin cortical lamella called subchondral bone plate. It is the organic component of the SB, and consists mainly of collagen type I, proteoglycan, glycosaminoglycans, and water (Madry et al. 2010; Bian et al. 2016; Stewart and Kawcak 2018).

The overall function of the SB is to protect the overlying cartilage and distribute forces that are generated through locomotion (Radin and Rose 1986; Stewart and Kawcak 2018). SB is highly responsive to mechanical loading and has the capability to react promptly to training and injury (Kawcak et al. 2001; Lieberman et al. 2001; Boudenot et al. 2014; Poulet et al. 2015). Meanwhile, the unique composition of subchondral bone allows for the gradual dispersion of the axial loads across the joint, as well as a minimized shear stress (Radin and Paul 1971; Radin et al. 1973; Imhof et al. 2000). The stiffness gradient between articular cartilage, $\mathrm{CC}$, and the subchondral bone plate is sharp, leading to an elasticity difference in mature species by a factor of 10-100 (Radin and Rose 1986; Li and Aspden 1997; Day et al. 2001; Hargrave-Thomas et al. 2015). However, the undulation of the tidemark and the cement line provides intimate contact between different zones of the subchondral bone, and transforms oblique loads into compressive and tensile forces (Radin and Rose 1986; Oegema et al. 1997; Imhof et al. 2000; Schultz et al. 2015). Subchondral bone is reportedly ten times more deformable compared to the cortex of long bones (Pathria et al. 2016; Stewart and Kawcak 2018). Hence, normal subchondral bone can dissipate about $30 \%$ of the loads inserted on the joint, while only 1-3\% of load forces are dissipated in cartilage (Radin and Paul 1970; Brown and Vrahas 1984). The remaining forces are attenuated by the supporting trabeculae, compact bone, 
and the joint capsule (Imhof et al. 2000; Oftadeh et al. 2015; Pereira et al. 2015). Branching distally from the cortical end-plate is the subchondral trabecular bone, which particularly provides elasticity and a crucial shock-absorbing ability during joint loading (Hayami et al. 1994; Li et al. 2013). It does not have a homogeneous composition, varies depending on the distance from the tidemark, demonstrates notable structural anisotropy, and contains sensory nerves, blood vessels, and bone marrow (Holopainen et al. 2008; Suri and Walsh 2012). Thus, to promote support and force distribution for the joint, the dynamic balance of functions between various layers of the joint is essential.

Even though the intimate model for the biomechanical crosstalk of the cartilage and the underlying SB is well-established, the biochemical crosstalk at the intersection of AC-SB is relatively uncharted (Li et al. 2013; Findlay and Kuliwaba 2016). It is suggested that the mediators produced from both cartilage and subchondral bone may pass from one tissue to another, which influences the functionality and homeostasis of the adjacent unit (Lyons et al. 2006; Yuan et al. 2014). It was demonstrated that leukotrienes, prostaglandins, and several growth factors were released in vivo by osteoblasts through the course of bone remodeling, and could reach the overlying AC (Lajeunesse and Reboul 2003). A synergic effect was observed, where the inflammatory and osteoclast stimulation factors released by the AC could lead to subchondral bone deterioration (Bellido et al. 2010; Henrotin et al. 2012). Hence, the traditional view that the calcified cartilage and the subchondral bone plate act as impenetrable barriers to soluble molecules is no longer agreed upon (Imhof et al. 1997; Yuan et al. 2014; Findlay and Kuliwaba 2016). Since the second half of the last century, few studies have reported smaller fractures or microchannels in the subchondral bone that seem to connect the lower margin of the articular cartilage with the medulla (Greenwald and Haynes 1969; Hodge and McKibbin 1969; Woods et al. 1970; Duncan et al. 1987; Milz S. and Putz 1994). Despite the potentially strong implications that these cartilage-bone marrow microchannel connectors (CMMC) could have on bone-cartilage homeostasis, the current data on this feature consists of isolated reports. A coherent picture is missing. In what follows, a concentrated review on the so far-described evasive microarchitectural characteristics of the SB is provided, which includes different species such as humans, with discussions regarding potential physiological functions of this anatomical feature. 


\subsection{SB microchannels in animal models}

In 1950, Holmdahl and Ingelmark conducted one of the first examinations of the subchondral bone's microchannel system in various joints (i.e. shoulder, elbow, hip, and knee) of 10-months-old rabbits (Holmdahl and Ingelmark 1950). The surfaces of these channels were divided into two categories: (1) Wide (ampulla-like) contacts, where the medulla rested on the cartilage within a relatively large area, and (2) canal-like (dendritic) contacts, where offshoots of the medullary cavity had immediate contiguity with the basal surface of the cartilage through a comparatively compact bone (Table 1; Holmdahl \& Ingelmark). They concluded that the direct contact between the cartilage and the medullary cavity was an isolated incidence in small parts of the subchondral end-plate where no calcified cartilage was found (Holmdahl and Ingelmark 1950). It was later confirmed that in mature rabbits, the $\mathrm{AC}$ of the femur was separated from the marrow cavity by calcified cartilage, while in immature rabbits, where the capital epiphysis was still growing, vascular channels extended into the articular cartilage (Hodge and McKibbin 1969). The proximal humeral chondroepiphysis of newborn rabbits was shown to be well-vascularized by a cluster of glomeruli that had a wide variety of sizes and shapes, reportedly reflecting the metabolic needs of the cartilage (Wilsman and Van Sickle 1972). Based on these results, it was concluded that subchondral-driven nutrition of the cartilage only plays a role in growing animals and that the nutritional contribution of the synovial fluid becomes more important with increasing age (Hodge and McKibbin 1969; Wilsman and Van Sickle 1972). Likewise, studies on blood flow distribution in the bones of the hind legs of ten- and twenty-week-old pigs revealed a significant ageassociated decline of the blood flow rate in the femoral, patellar and metatarsal cartilage (Nakano et al. 1986). On the other hand, several perfusion-based studies on animal joints have been conducted to test if molecular trafficking between SB and AC can occur. Using a photobleaching-induced fluorescence loss imaging technique, it was found that small molecules such as sodium fluorescein (376 Da) could be diffused between the marrow cavity and the cartilage of the murine distal femur (Pan et al. 2009). Similarly, experiments performed on healthy horse metacarpal joints revealed that the tidemark and CC are permeable to low-molecular-weight solutes (Arkill and Winlove 2008). Hence, the subchondral microchannel system exists in various animal models, connects the uncalcified articular cartilage to marrow spaces, and thus provides a molecular diffusion pathway that can have metabolic effects (Lyons et al. 2006). 


\subsection{Microarchitecture of SB in healthy human knee joint}

A scanning electron microscopy (SEM) study on non-arthritic knee joints showed that the SB was perfused with perforations, while their local density was higher sub-meniscally in the medial tibial plateau compared to the central area that was not covered by the meniscus (Duncan et al. 1987). A propensity for larger holes has been described peripherally and posteriorly on the medial plateau. In the lateral plateau, however, they observed a reversed pattern, where a higher number of holes was found in the central region than in its submeniscal counterpart (see Table 1; Duncan et al.). A mean diameter of $89 \mu \mathrm{m}$ was measured for the perforations of the medial and lateral plateaus, while no inter-plateau differences were found for the morphology of the perforations (Duncan et al. 1987). In another study, three types of cavities that penetrated the CC of the human knee joint were identified and categorized based on size as well as distinct anatomical features (Table 1; (Clark 1990)). The largest cavities (> $100 \mu \mathrm{m})$ were irregular in shape, and contained fat or marrow cells. It was reported that these large cavities are mostly located in the center and not in the peripheral and posterior regions of the plateau (Clark 1990). The second type of cavity was medium-sized canals $(30-70 \mu \mathrm{m})$ that branched out from the large marrow spaces, had finger-like appearances, and occasionally contained blood vessels. The third type of cavity was the smallest (diameter $=10-30 \mu \mathrm{m})$ and held one or two erythrocyte-containing blood vessels. The local density of these small vascular cavities was associated with the thickness of the subchondral plate. Many vascular perforations were observed in the centrally-thick region of the tibial plateau, but few were detected in peripheral areas where the subchondral bone was thin (Clark 1990). Similar observations were made by Milz and Putz, where a branching pattern of CMMC (mean diameter $=30 \mu \mathrm{m}$; see Table 1 for details) were identified in the central and proximal areas of the tibial plateau. In this region, the thickest SBP meets the thickest articular cartilage (Milz S. and Putz 1994). In the peripheral regions of the tibial plateau, in which the thinnest subchondral plate is located, larger hole-like gaps have been described, the number of which, however, was smaller compared to the centrally located vascular canals (Milz S. and Putz 1994). In places with an extremely thin subchondral plate, where the overlying cartilage was essentially in direct contact with the medulla, larger cavities (> $80 \mu \mathrm{m})$ could be found. Such large cavities were found particularly in the outermost joint areas near the edge with only a very thin cartilaginous coating of the joint surfaces (Milz S. and Putz 1994). 
Table 1. Characteristics of SB perforations in animal models and the human knee joint

\begin{tabular}{|c|c|c|c|c|c|c|c|c|c|c|c|}
\hline Study & $\begin{array}{l}\text { Tech } \\
\text { niqu }\end{array}$ & $\begin{array}{c}\text { Specie } \\
\text { s/Age }\end{array}$ & $\begin{array}{c}\text { Type of } \\
\text { the }\end{array}$ & Joint & \multicolumn{3}{|c|}{$\begin{array}{l}\text { Size of the perforation } \\
(\mu \mathrm{m})\end{array}$} & \multicolumn{4}{|c|}{$\begin{array}{l}\text { Number of the } \\
\text { perforations }\end{array}$} \\
\hline \multirow{11}{*}{$\begin{array}{l}\text { (Holm } \\
\text { dahl } \\
\text { and } \\
\text { Ingel } \\
\text { mark } \\
1950)\end{array}$} & \multirow[t]{11}{*}{$\begin{array}{l}\text { Histo } \\
\log y\end{array}$} & \multirow{11}{*}{$\begin{array}{l}\text { Rabbit } \\
\text { / 10- } \\
\text { month }\end{array}$} & \multirow{11}{*}{$\begin{array}{l}\text { Contacts } \\
\text { between } \\
\text { the AC } \\
\text { and the } \\
\text { marrow } \\
\text { cavity }\end{array}$} & $\begin{array}{c}\text { prox. } \\
\text { Humerus }\end{array}$ & $\begin{array}{l}\text { Canal- } \\
\text { like }\end{array}$ & \multicolumn{2}{|c|}{ Wide } & \multicolumn{2}{|c|}{ Canal-like $^{1}$} & \multicolumn{2}{|c|}{ Wide $^{1}$} \\
\hline & & & & & $\begin{array}{c}16.74 \pm \\
0.58\end{array}$ & \multicolumn{2}{|c|}{$51.6 \pm 3.2$} & \multicolumn{2}{|c|}{47} & \multicolumn{2}{|c|}{53} \\
\hline & & & & Scapula & $\begin{array}{c}17.8 \pm 1 \\
4\end{array}$ & \multicolumn{2}{|c|}{$40.1 \pm 2.0$} & \multicolumn{2}{|c|}{54} & \multicolumn{2}{|c|}{46} \\
\hline & & & & $\begin{array}{c}\text { Dist. } \\
\text { Humerus }\end{array}$ & $\begin{array}{c}12.08 \pm \\
0.44\end{array}$ & \multicolumn{2}{|c|}{$30.0 \pm 3.1$} & \multicolumn{2}{|c|}{98} & \multicolumn{2}{|c|}{2} \\
\hline & & & & $\begin{array}{l}\text { Prox. } \\
\text { Radius }\end{array}$ & $\begin{array}{c}11.41 \pm \\
0.88\end{array}$ & \multicolumn{2}{|c|}{25.8} & \multicolumn{2}{|c|}{92} & \multicolumn{2}{|c|}{8} \\
\hline & & & & $\begin{array}{l}\text { Prox. } \\
\text { Ulna }\end{array}$ & $\begin{array}{c}14.5 \pm 1 \\
1\end{array}$ & \multicolumn{2}{|c|}{45.9} & \multicolumn{2}{|c|}{90} & \multicolumn{2}{|c|}{10} \\
\hline & & & & $\begin{array}{l}\text { Prox. } \\
\text { Femur }\end{array}$ & $\begin{array}{c}15.28 \pm \\
0.65\end{array}$ & \multicolumn{2}{|c|}{$41.9 \pm 2.9$} & \multicolumn{2}{|c|}{70} & \multicolumn{2}{|c|}{30} \\
\hline & & & & $\begin{array}{l}\text { Acetabul } \\
\text { um }\end{array}$ & $\begin{array}{c}16.7 \pm 2 \\
0\end{array}$ & \multicolumn{2}{|c|}{$45.81 \pm 0.74$} & \multicolumn{2}{|c|}{22} & \multicolumn{2}{|c|}{78} \\
\hline & & & & Patella & $\begin{array}{c}13.8 \pm 2 \\
4\end{array}$ & \multicolumn{2}{|c|}{$34.6 \pm 2.9$} & \multicolumn{2}{|c|}{84} & \multicolumn{2}{|c|}{16} \\
\hline & & & & $\begin{array}{l}\text { Dist. } \\
\text { Femur }\end{array}$ & $\begin{array}{c}15.7 \pm 1 \\
8\end{array}$ & & & & 6 & & 4 \\
\hline & & & & $\begin{array}{l}\text { Prox. } \\
\text { Femur }\end{array}$ & 17 & 45.5 & \pm 1.4 & & & & 7 \\
\hline $\begin{array}{l}\text { (Liu et } \\
\text { al. } \\
2011 \text { ) }\end{array}$ & SEM & $\begin{array}{c}\text { Huma } \\
\text { n } \\
\text { (adult) }\end{array}$ & $\begin{array}{l}\text { Protrusi } \\
\text { on-pores } \\
\text { of the } \\
\text { SB }\end{array}$ & $\begin{array}{l}\text { Distal } \\
\text { femur }\end{array}$ & $\begin{array}{l}\text { At AC- } \\
\text { CC } \\
\text { interfac } \\
\mathrm{e}\end{array}$ & $\begin{array}{r}\text { At CC } \\
\text { inte }\end{array}$ & $\begin{array}{l}-\mathrm{SBP} \\
\text { face }\end{array}$ & & & & \\
\hline & & & & & $\begin{array}{c}17.0 \\
(1.98- \\
114.25)\end{array}$ & $\begin{array}{r}34.1 \\
100\end{array}$ & $\begin{array}{l}3.66- \\
96)\end{array}$ & & & & \\
\hline (Dunc & SEM & Huma & Perforati & Tibial & $89(\mathrm{r}$ & ng: $31-$ & 59) & & $\mathrm{lial}^{2}$ & & $\mathrm{eral}^{2}$ \\
\hline $\begin{array}{c}\text { an et } \\
\text { al. } \\
\text { 1987) }\end{array}$ & & $\begin{array}{c}\text { n / 43- } \\
95 \\
\text { years }\end{array}$ & $\begin{array}{l}\text { ons or } \\
\text { holes } \\
\text { through }\end{array}$ & plateau & & & & $\begin{array}{c}\text { cent } \\
\text { ral }\end{array}$ & $\begin{array}{l}\text { Sub- } \\
\text { men } \\
\text { iscal }\end{array}$ & $\begin{array}{c}\text { cent } \\
\text { ral }\end{array}$ & $\begin{array}{c}\text { Sub- } \\
\text { menis } \\
\text { cal }\end{array}$ \\
\hline & & & the SB & & & & & 0.94 & 1.69 & 1.35 & 0.85 \\
\hline (Milz & $\begin{array}{l}\text { Micr } \\
\text { oradi }\end{array}$ & Huma & $\begin{array}{c}\text { SB } \\
\text { perforati }\end{array}$ & $\begin{array}{l}\text { Proximal } \\
\text { tibia }\end{array}$ & $\begin{array}{l}\text { Chanel- } \\
\text { like }\end{array}$ & form & tions & & & & \\
\hline $\begin{array}{c}\text { Putz } \\
1994)\end{array}$ & $\begin{array}{l}\text { ogra } \\
\text { phy }\end{array}$ & $\begin{array}{c}84 \\
\text { years }\end{array}$ & ons & & $\begin{array}{c}30(20- \\
45)\end{array}$ & $82(4$ & $-145)$ & & & & \\
\hline $\begin{array}{l}\text { (Clark } \\
1990)\end{array}$ & SEM & $\begin{array}{c}\text { Huma } \\
\text { n / 17- } \\
72 \\
\text { years } \\
\text { Dog / } \\
\text { matur } \\
\text { e }\end{array}$ & $\begin{array}{c}\text { SB } \\
\text { perforati } \\
\text { ons }\end{array}$ & $\begin{array}{l}\text { Tibial } \\
\text { plateau, } \\
\text { patellae, } \\
\text { femoral } \\
\text { condyle }\end{array}$ & Wide & $\begin{array}{l}\text { Cylin } \\
\text { drical } \\
\text { canals }\end{array}$ & $\begin{array}{c}\text { Vasc } \\
\text { ular } \\
\text { canal } \\
\text { s }\end{array}$ & & & & \\
\hline & & $\begin{array}{c}\text { Rabbit } \\
/ \\
\text { matur } \\
\mathrm{e}\end{array}$ & & & 100 & $30-70$ & $10-30$ & & & & \\
\hline
\end{tabular}

SB: subchondral bone; AC: articular cartilage; CC: calcified cartilage; SBP: subchondral bone plate; SEM: scanning electron microscopy; ${ }^{1}$ The percentile number of contacts; ${ }^{2}$ Number of perforations per $\mathrm{mm}^{2}$ 
A schematic representation of the SB perforations in the human knee joint is depicted in Figure 2.
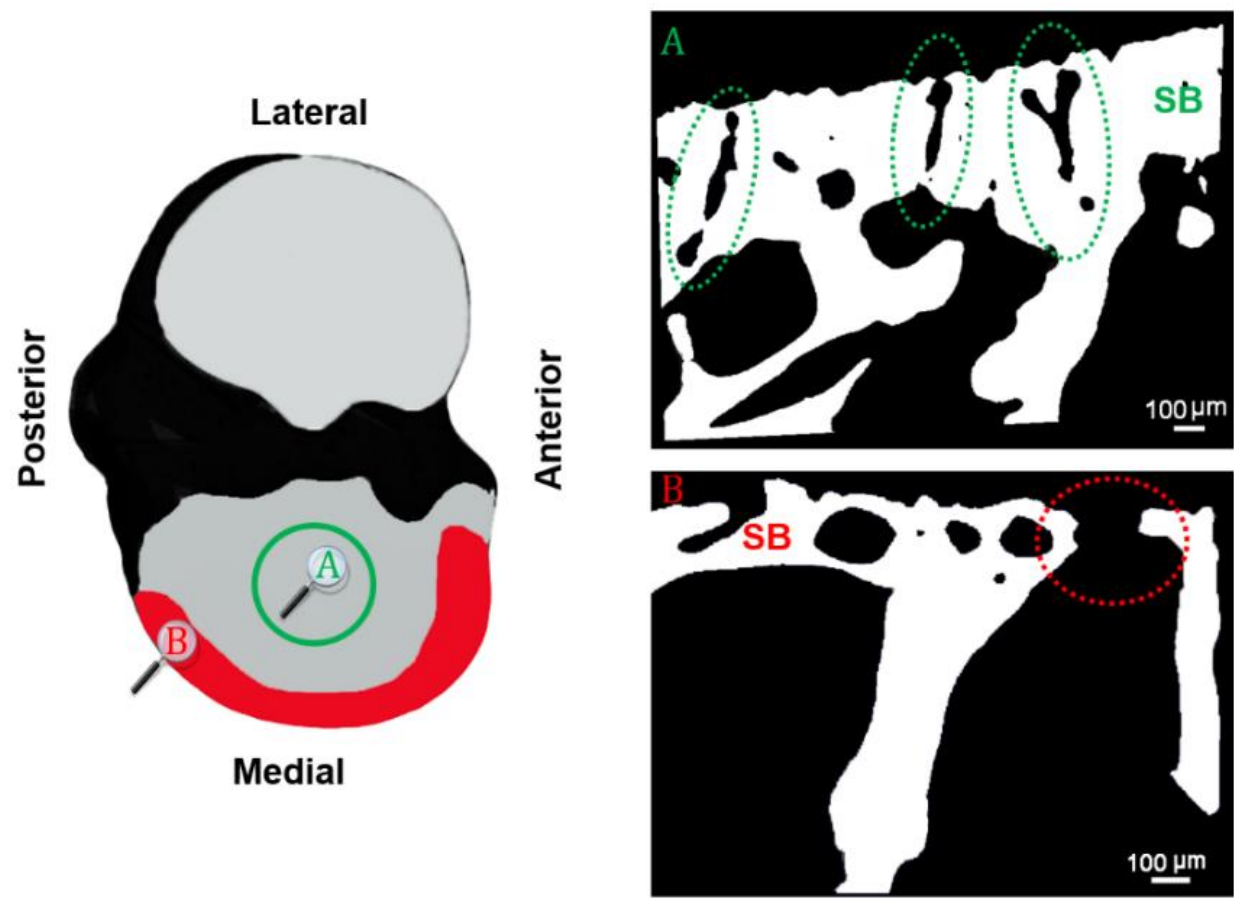

Figure 2. A schematic model for the SB microchannels in human tibial plateau. Based on the work of (Milz S. and Putz 1994), (A) in the central area of the medial condyle (roughly encircled in green), dendric channels are branched into the tidemark while in peripheral regions (B; red), larger hole-like gaps connect the AC-SB junction to the medullary cavity.

\subsection{CMMC in healthy human femoral head}

In healthy human femoral heads, the presence of a connective pathway from the marrow cavity to the articular cartilage was reported for the first time by Greenwald and Haynes in 1969 (Greenwald and Haynes 1969). A fluorescent dye that was inserted approx. 5 millimeters below the subchondral bone could be detected after 24 hours of incubation at the boundary between calcified cartilage and articular cartilage. After 48 hours, the dye penetrated the articular cartilage and reached the articular surface. The greatest intensity and the deepest dye penetration were detected in areas where subchondral perforations were near or in contact with the articular cartilage (Greenwald and Haynes 1969). A year later, these findings were confirmed using a technique based on stereoscan electron microscopy (Mital and Millington 1970). Even though the cellular material around the walls of the channels could not be precisely determined, many branched cavities were observed that originated from cancellous bone and reached in human hip joints as far as the basal layer of the articular cartilage (Mital and Millington 1970). In an attempt to 
quantify the vascular penetrations in the healthy adult human femoral head, the "breaks" or "defects" of the subchondral bone were classified into three types. "Type A" denotes the presence of vascularized fibrous tissue, as well as a direct contact between the tissue and the articular cartilage. "Type B" contains typical bone structures, and "Type C" is surrounded by small pieces of woven bone that were either unvascularized or contained a very tiny vascular channel (Woods et al. 1970). It was observed that the number of all types of CMMC was higher in the superocentral regions than at the periphery. Moreover, the density of vascular channels (Type A and B) directly under the stress zones of the femoral head was higher than under the less stressed areas. Interestingly, the vascularity index (the ratio of vascular to total number of channels) steadily declined with age, even though the total number of defects showed no age-related changes over the course of adult life (Woods et al. 1970). However, in a further investigation, two age-dependent phases were diagnosed for the vascularity changes of the SB and $\mathrm{CC}$ in adult human femoral heads: (1) Up to the age of 70 it was found that the number of vascular microchannels per unit area decreased by $20 \%$ compared to adolescents. (2) After the age of 70 , the vascularity of SB increased (Lane et al. 1977). Vessels in the generally loaded areas (superior portion) of the femoral head were $25 \%$ more frequent than in the non-exposed areas (inferior portion; Table 2; Lane et al.). The degree of active remodeling was measured through a morphological identification of various stages of remodeling activity (i.e. resorptive phase, new bone deposition, inactive phase).

Table 2. Characteristics of SB perforations in the human femoral head

\begin{tabular}{|c|c|c|c|c|c|c|}
\hline Study & Technique & $\begin{array}{c}\text { Species/Ag } \\
\text { e }\end{array}$ & $\begin{array}{l}\text { Type of } \\
\text { the }\end{array}$ & Joint & \multicolumn{2}{|c|}{$\begin{array}{c}\text { Number of the } \\
\text { perforations }\end{array}$} \\
\hline \multirow[t]{2}{*}{$\begin{array}{l}\text { (Lane et } \\
\text { al. 1977) }\end{array}$} & \multirow[t]{2}{*}{ Microscopy } & \multirow{2}{*}{$\begin{array}{c}\text { Human } \\
(15-93 \\
\text { years })\end{array}$} & \multirow[t]{2}{*}{$\begin{array}{l}\text { Vascular } \\
\text { vessels }\end{array}$} & \multirow[t]{2}{*}{$\begin{array}{l}\text { Femoral } \\
\text { head }\end{array}$} & $\begin{array}{c}\text { Superior } \\
1\end{array}$ & Inferior1 \\
\hline & & & & & 6.25 & 4.69 \\
\hline \multirow{2}{*}{$\begin{array}{l}\text { (Meachi } \\
\text { m and } \\
\text { Allibone } \\
\text { 1984) }\end{array}$} & \multirow[t]{2}{*}{$\begin{array}{c}\text { Photomicrograp } \\
\text { hy }\end{array}$} & \multirow{2}{*}{$\begin{array}{c}\text { Human } \\
(19-86 \\
\text { years) }\end{array}$} & \multirow{2}{*}{$\begin{array}{l}\text { defects in } \\
\text { contact } \\
\text { with the } \\
\text { tidemark. }\end{array}$} & \multirow[t]{2}{*}{$\begin{array}{l}\text { Femoral } \\
\text { head }\end{array}$} & Zenith & $\begin{array}{l}\text { Inferomedi } \\
\text { al }\end{array}$ \\
\hline & & & & & 7.7 & 1.2 \\
\hline \multirow[t]{2}{*}{$\begin{array}{l}\text { (Berry et } \\
\text { al. 1986) }\end{array}$} & \multirow[t]{2}{*}{ Histology } & \multirow[t]{2}{*}{$\begin{array}{l}\text { Adult } \\
\text { Human }\end{array}$} & \multirow{2}{*}{$\begin{array}{l}\text { subchondr } \\
\text { al defects } \\
\text { in the talar } \\
\text { dome }\end{array}$} & \multirow[t]{2}{*}{$\begin{array}{l}\text { Tibiotala } \\
\text { r joints }\end{array}$} & $\begin{array}{c}\text { Tibial } \\
\text { contact } \\
\text { area2 }\end{array}$ & $\begin{array}{c}\text { Fibular } \\
\text { contact } \\
\text { area2 }\end{array}$ \\
\hline & & & & & 3.90 & 2.76 \\
\hline
\end{tabular}


It has been shown that active remodelling decreases $50 \%$ from adolescence to the age of 70 , and then rises again to levels comparable to those found at young ages (Lane et al. 1977). The findings of Woods et al. with regard to the three types of focal contacts between articular cartilage and marrow cavity has been verified elsewhere (Meachim and Allibone 1984). The number of focal contacts that were in contiguity with the uppermost surface of the tidemark was consistently higher at the superocentral region compared to the inferomedial portion of the femoral head (Table 2; Meachim \& Allibone) (Meachim and Allibone 1984).

\subsection{Connective microfeatures in other human joints}

An investigation of the vascularity and remodeling of the calcified cartilage and the subchondral bone endplate of adult human humeral heads over the course of life showed that the local density of the vascular canals decreases by $15 \%$ up to the age of 60 years. In the period from 60 to 90 years, however, no age-related changes were observed (Lane et al. 1977). In all age groups, the number of vessels crossing the calcified cartilage layer was $15 \%$ higher in the loaded areas. A direct correlation was detected between the local density of the vascular channels and the degree of active bone remodelling, with a similar remodelling pattern to that in the femur, being reduced by $30 \%$ up to the age of 60 and thereafter increasing to values that came close to those at young ages (Lane et al. 1977). The intact adult human tibiotalar joints were also evaluated and three types of subchondral defects (corresponding to the work of Woods et al.) were measured for the areas of tibial and fibular contact (Berry et al. 1986). The number of defects per centimeter of articular surface was significantly higher in the tibial contact area compared to its fibular counterpart (Table 2; Berry et al. (Berry et al. 1986)). For both areas of contact, the greatest local defect density was centrally located on the talar dome and concentrated more on the posterior edge than on the anterior side. The vascularized defects were observed more prominently in the tibial contact zone compared to the fibular zone, and were highly concentrated in the central portion of the talar dome. Although the number of non-vascularized defects was overall higher than that of vascularized defects, no differences were found between the two contact areas (Berry et al. 1986). It was also shown that a fluorescent dye could be dispersed through the talar domes of most specimens after 48 hours of perfusion time, with the intensity being highest in central areas of the talus and lower near the corners (Berry et al. 1986). In addition, the diffusivity of the upper and lower end-plates of human lumbar vertebrae was examined (Nachemson 
et al. 1970). It was observed that the upper and lower central portions of the endplates were permeable in $87-89 \%$ of the endplates of the $\mathrm{L}_{3}$ and $\mathrm{L}_{4}$ disks examined. Histological examinations showed an association between the permeability of the end-plate and the presence of "vascular buds" (vascular projections with ampulla-like contacts between the medullary canal of the vertebral body and the hyaline cartilage of the intervertebral disc's endplate). In contrast, it has been reported that the lateral part of the endplates is normally impermeable (Nachemson et al. 1970).

\subsection{Hypothesized functions of the CMMC}

Several theories have been proposed to explain the presence of microchannels within the subchondral bone. Since articular cartilage is avascular, it is envisioned that they may be molecular diffusion pathways that provide nutrition to the deeper chondrocytes adjacent to the tidemark (Meachim and Allibone 1984; Duncan et al. 1987; Lyons et al. 2006). Moreover, it was observed that some microchannels have a wall structure that does not correspond to the structure of typical, fully-trained osteons (Milz S and Putz 1994; Milz S. and Putz 1994). Such classic osteons are mostly accumulated in the shafts of long bones and inhibit mechanical deformability (Carnelli et al. 2013; Lefèvre et al. 2019). Thus, the microchannels may be directly responsible for the significant deformability of the subchondral bone compared to the cortical shaft of the long bones. Additional biomechanical functionality of the SB perforations is reportedly reflected in their slightly helical shape, which may allow for small compressions of the calcified cartilage and prevent fractures at the osteochondral junction (Lyons et al. 2006). It is also suggested that the differently-shaped perforations within the SB may not only have nutritive function, but also serve as passages for the interstitial fluid movement between the cartilage and the medullary cavity (Milz S. and Putz 1994; Imhof et al. 1997; Hwang et al. 2008). Thus, the SB microchannels may offer a hydromechanical functionality, where the pressure of the deeper layers of the AC is relayed via fluid shifts. On the other hand, SB perforations occurring in old populations have been described as an occasional occurrence associated with pathological processes in arthropathy (Green et al. 1970; Oettmeier et al. 1989). According to this viewpoint, the vascular channels in the SB of adult joints are signs of a remodelling process, which later manifests as angiogenesis at the osteochondral junction (Lane et al. 1977; Clark 1990). 


\subsection{The aim of the thesis}

The pathophysiology of OA remains elusive to this date, and while there have been successes in preclinical and early clinical studies, no definite cure is still available (Liu et al. 2018; Van Spil et al. 2019). Nevertheless, as our comprehension of the underlying OA pathophysiology grows, the notion that $\mathrm{OA}$ is only a disease of $\mathrm{AC}$ has become outdated, with osteochondral unit emerging as a focal point of OA research (Stewart and Kawcak 2018). Subchondral bone, in particular, has been recognized as a critical contributing factor to the initiation and progression of OA. As discussed earlier, AC and SB are intimately interconnected and have extensive biomechanical and biochemical crosstalk (Lories and Luyten 2011). SB is also heavily innervated by sensory nerves that are associated with pain (Suri et al. 2007). Interestingly, recent results have linked joint pain with subchondral osteoclast activity and have inferred that bone remolding might precede severe cartilage degeneration (Nwosu et al. 2017).

As all evidence point to the fact that the state of cartilage depends heavily on the density and (micro)architecture of the SB (Bobinac et al. 2003), evaluation of the SB microstructure becomes vital for a deeper understanding of its intimate interrelationship with cartilage, for unravelling OA pathogenesis, and for developing future clinical intervention (Liu et al. 2018). However, most studies so far have concentrated on the role of bone mineral density and (to a lesser extent) the trabecular microstructure (FunckBrentano and Cohen-Solal 2015; Chu et al. 2020; Renault et al. 2020). Very few studies have focused on the microstructure of the calcified cartilage and the subchondral bone plate, and the significance that they might have for different health-stages of the joint (Deng et al. 2016; Kauppinen et al. 2019). In particular, there is nearly a 20-year gap between the most recent reports that have focused on the canalicular connections between the AC and SB (Holmdahl and Ingelmark 1950; Woods et al. 1970; Clark 1990). Studies on the SB perforations have been scarce and sporadic, even though a precise model of their microarchitecture and functionality may potentially revolutionize our understanding of the joint physiology and pathophysiology. Moreover, there are some contradictory observations regarding the SB microchannel system, which prevents the formation of a unified hypothesis regarding its functionality. For example, it was asserted that microchannels rarely transverse through the calcified cartilage in the tibial plateau (Clark and Huber 1990), while other examinations confirmed numerous perforations in the calcified cartilage layer of the tibial plateau (Madry et al. 2010). Likewise, some studies have reported that these perforations are occasional and intermittent in older people 
(Green et al. 1970), while others have found frequent and regular canals in old populations (Milz S. and Putz 1994). Additionally, the differences in the morphology have sometimes been ascribed to the health-state of the overlying cartilage (normal vs. partially eroded vs. fully eroded) (Hwang et al. 2008), while in other cases, these differences are found in different locations within the same joint of healthy samples (Clark 1990; Milz S. and Putz 1994). The first reason for this contradictory evidence might be a lack of spatial distribution representation of these perforations, due to the commonly-used 2D approaches (i.e. scanning electron microscopy, photomicrography). 3D representation of the osteochondral junction has so far been reported in only one case, where sequential micrographs of haematoxylin and eosin stainings were overlaid (Lyons et al. 2006). The second reason may be overlooking microstructural variances in different locations of the same tissue. We propose that the microarchitecture of the SB, and specifically its evasive anatomical features, merit closer examinations with the aid of improved imaging techniques. Hence, the main objective of this thesis is to shed light on the role of the SB (micro)architecture in joint physiology and pathophysiology by developing methods based on high-resolution imaging to quantitatively describe the SB microarchitecture in four different models. Namely, models of maturation, adult physiological human joint, early OA human joint, and a traumatic OA pathophysiology model. In total, this thesis consists of four main studies.

First, it was examined how pure maturation can alter the SB microstructure. Since the majority of pre-clinical research on the role of the SB has been dedicated to comparative evaluations of healthy vs. early osteoarthritis or early OA vs. late-stage OA cases (Kamibayashi et al. 1995; Bobinac et al. 2003; Yan et al. 2014; Chevrier et al. 2015; Huang et al. 2017), studies on the microstructure of normal healthy SB and how they change from young age to early adulthood has been surprisingly rare (Martinelli et al. 2002; Dingemanse et al. 2017; Lemirre et al. 2020). Hence, the SB microarchitecture of the medial condyle of the femur in calves (three months) and cattle (18 months) was examined. It was hypothesized that different layers of the SB (i.e. calcified cartilage, and subchondral bone plate) reveal a marked transformation in terms of microstructure and mineral density. Additionally, the aim was to provide the first high-resolution 3D representation of the SB microchannel system, and assess possible age-related changes.

Second, the previous methodology was expanded to adult (age: 40-60) physiological human samples. A high-resolution 3D profile of the subchondral bone in healthy human femoral heads were created, the SB microchannels were quantified with newly-defined 
morphological indices, and the results were correlated to the overlying cartilage. Here, it was hypothesized that the characteristics and the spatial distribution of these CMMC are influenced by local forces on the joint. Likewise, I wanted to see if a recurring pattern could be observed for the distribution of the SB microchannels, whether I can provide answers to some of the contradictory observations regarding the nature of the SB microchannels, and if the results can be integrated into a revised anatomical model for the joint.

Third, to provide further information about possible relationships between the SB microchannels and the health-state of the joint, I translated the established methodology to early osteoarthritic human samples. Local areas of moderate cartilage softening and fibrillation were identified (typical of early OA) on adult human femoral heads and highresolution scanning was performed. The results from the second study were regarded as the control group. It was hypothesized that the characteristics of the underlying SB, including its microarchitecture, are changed in early OA compared to the healthy, control cohort. Based on different theories about the functionality of the SB microchannels, it was evaluated if/how these microstructures can be included in pathophysiological models of the joint, and if/how they can be used to design novel diagnostic and treatment strategies.

Fourth, in a traumatic murine model of surgical destabilization of the medial meniscus (DMM), the early and late (micro)structural changes of the subchondral bone were assessed. The progression of SB degenerative alterations in the calcified cartilage layer and the medial epiphysis of the knee joint were analyzed, and compensatory adaptations of the joint after the OA induction (i.e. osteophyte formation, changes in the medial and lateral condyles length, and ectopic bone formation in meniscus) were quantified with progression from early OA to late-stage OA. Additionally, the influence of sensory neuropeptides substance P (SP) and alpha-calcitonin gene-related peptide ( $\alpha$ CGRP) on the (subchondral) bone microarchitecture was evaluated two- and eight-weeks post trauma. As SP- and $\alpha \mathrm{CGRP}$-containing nerve fibers are frequently found in bone adjacent to the blood vessels that target periosteum, subchondral trabecular bone, and bone marrow, it is hypothesized that the absence of both sensory neuropeptides would be detrimental to the bone homeostasis. 


\section{Materials and Methods}

\subsection{Biopsy and preparation of samples}

\subsubsection{Bovine model}

The knee joints of three-month-old male calves $(n=3)$ and 18-month-old $(n=3)$ male cattle were obtained and dissected on the day of slaughter at an abattoir. The surrounding soft tissue was cut off to expose the joint surface. From each medial condyle of femur, cartilage-bone cylinders were extracted with an inner diameter of $6 \mathrm{~mm}$, accounting to a sum of 18 cartilage-bone biopsies. With the aid of a template grid, the location of measuring points was standardized, taking into consideration the normalized size of each joint. For each type of analysis, samples that were punched out from the same anatomical location were used. Hence, cartilage-bone cylinders that were extracted from K1, K2, and K3 were respectively used for micro-CT evaluation, histomorphometrical analysis, and energy-dispersive X-ray spectroscopy. The results of the latter are not presented in this dissertation (Figure 3).

The cartilage-bone biopsies described in this section were obtained and prepared by Dr. Thomas Winkler and Lia Sabrina Schenk in cooperation with the Institute of Biomechanics, Technical University Hamburg-Harburg.
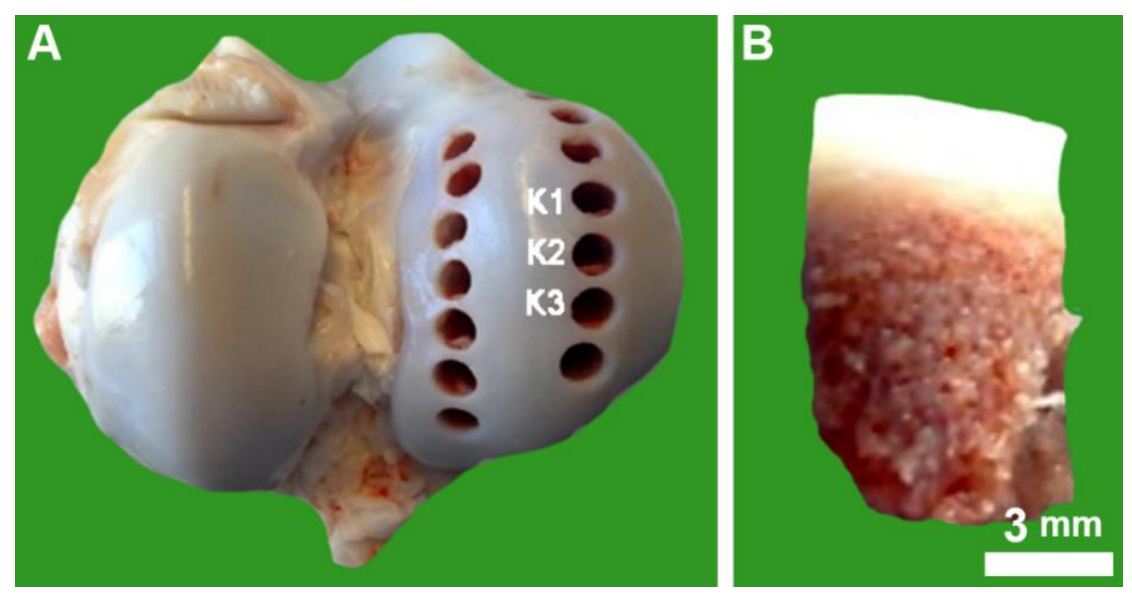

Figure 3. Preparation of cartilage-bone biopsies from the bovine bone. (A) On the contact area of the femoral condyle, bone extracts from the $\mathrm{K} 1, \mathrm{~K} 2$, and $\mathrm{K} 3$ measuring points were evaluated by micro-CT, histomorphometry, and EDX analysis, respectively. An exemplary osteochondral biopsy is shown in (B)

\{SOURCE: (Schenk 2010; Winkler 2012), modified\} 


\subsubsection{Healthy and early osteoarthritic human femoral heads}

The human femurs used in this study were granted by the anatomical gift programme of the Medizinische Hochschule Hannover (MHH). Prior to death, all donors gave their written informed consent that their cadavers be donated for research purposes. Femurs were embalmed in a $10 \%$ solution of neutral formalin buffered, wrapped individually in plastic bags and stored at $+4^{\circ} \mathrm{C}$ in sealed containers. No information was provided regarding the medical history, sex, age, and the medical history of the donors due to the anonymity of the MHH gift programme. Therefore, the sex and the biological ages at death were determined by a DNA-based methodology and a microscopic investigation, respectively.

\subsubsection{Outerbridge classification of the femurs}

The OA status was not available for any of the subjects. Hence, the femurs were graded independently by three orthopaedic surgeons according to the Outerbridge classification for chondral lesions (Outerbridge 1961; Slattery and Kweon 2018). Femurs that manifested healthy femoral head characteristics (mean Outerbridge grade $<0.5$ ) were considered intact, while the early OA samples were defined when the mean Outerbridge grade of the femoral heads were $1.5 \leq$ Grade $\leq 2.5$. The chondral lesions in early osteoarthritis were predominantly characterized by partial-thickness defects, fibrillations, and vertical fissures that extended into the mid-zone of the articular cartilage (de Windt et al. 2013; Slattery and Kweon 2018). Noticeable artifacts (e.g. iatrogenic erosions, tears, or elevations) and marked deformities such as coxa vara or coxa valga were exclusion criteria. After Outerbridge classification, several samples that met these criteria were shortlisted for further sex and age determination.

\subsubsection{Sex determination}

Due to the low quality of DNA in formalin-fixed tissues, modified methods of extraction are more useful in order to yield DNAs of long fragmented quality (Campos and Gilbert 2012). Based on Campos and Gilbert's protocol, the DNA-protein crosslinks can be broken by a heat-assisted technique (Campos and Gilbert 2012). In short, the fixed tissue powder was incubated in an alkali buffer solution $(0.1 \mathrm{M} \mathrm{NaOH}$ with $1 \% \mathrm{SDS})$ and heated to $120^{\circ} \mathrm{C}$ for $25 \mathrm{~min}$ using an autoclave. Nucleic acids were then separated from the crosslinked proteins by a 25:24:1 phenol: chloroform: isoamyl alcohol solution and purified in further procedures. The DNA concentration was subsequently calculated by a DeNovix 
DS-11 FX+ spectrophotometer (Wilmington, USA). The absorbance ratios $(1.8<$ $\mathrm{A}_{260} / \mathrm{A}_{280 / 230}<2.0$ ) signified DNAs of excellent quality (Table 3). The DNAs were stored at $-20^{\circ} \mathrm{C}$ before further processing.

Table 3. Quality characteristics of the extracted DNAs from healthy and early OA femurs

\begin{tabular}{|c|c|c|c|c|c|c|}
\hline Subjects & OA status & $\begin{array}{c}\text { Concentration } \\
(\mathrm{ng} / \mu \mathrm{L})\end{array}$ & $\mathrm{A}_{260} / \mathrm{A}_{280}$ & $\begin{array}{c}\mathrm{A}_{260} / \mathrm{A}_{280} \\
\text { Alert }\end{array}$ & $\mathrm{A}_{260} / \mathrm{A}_{230}$ & $\begin{array}{c}\mathrm{A}_{260} / \mathrm{A}_{230} \\
\text { Alert }\end{array}$ \\
\hline 1 & healthy & 377.28 & 1.84 & Met criteria & 1.83 & Met criteria \\
\hline 2 & healthy & 432.08 & 1.9 & Met criteria & 2.03 & Met criteria \\
\hline 3 & healthy & 1376.061 & 1.94 & Met criteria & 2.01 & Met criteria \\
\hline 4 & healthy & 108.214 & 1.92 & Met criteria & 2.14 & Met criteria \\
\hline 5 & healthy & 993.7 & 1.96 & Met criteria & 2.28 & Met criteria \\
\hline 6 & Early OA & 91.188 & 1.88 & Met criteria & 1.95 & Met criteria \\
\hline 7 & Early OA & 1276.039 & 1.93 & Met criteria & 2.03 & Met criteria \\
\hline 8 & Early OA & 4531.915 & 1.93 & Met criteria & 2.18 & Met criteria \\
\hline 9 & Early OA & 464.524 & 1.92 & Met criteria & 2.07 & Met criteria \\
\hline 10 & Early OA & 623.625 & 1.96 & Met criteria & 2.16 & Met criteria \\
\hline
\end{tabular}

A gonosomal sexing system (C. Roos 2010, unpublished data) based on polymerase chain reaction (PCR) was used to identify the sex of the several candidate subjects. The primers were designed to create a distinctive product at the $\mathrm{X}$-chromosome $(163 \mathrm{bp})$ and the $\mathrm{Y}$ chromosome (137 bp). PCR was performed in $50 \mu \mathrm{l}$ of master mixture, which incorporated $100 \mathrm{ng}$ of DNA solution, $0.5 \mu \mathrm{l}$ of each dNTP (Biozym, Hessisch Oldendorf, Germany), $2 \mu \mathrm{l}$ of each forward and reverse primers, $5 \mu$ l Buffer (Biozym, Hessisch Oldendorf, Germany), $0.25 \mu 1$ Taq DNA Polymerase (Biozym, Hessisch Oldendorf, Germany), and $39.25 \mu 1$ sterile ultrapure water. On a thermal cycler (SensoQuest Labcycler, Göttingen, Germany) and with the aid of a programme that comprised of denaturation at $95^{\circ} \mathrm{C}$ for $5 \mathrm{~min}$, followed by 40 cycles of denaturation $\left(95^{\circ} \mathrm{C}, 30 \mathrm{sec}\right)$, annealing $\left(56^{\circ} \mathrm{C}, 30 \mathrm{sec}\right)$ and primer extension $\left(72^{\circ} \mathrm{C}, 30 \mathrm{sec}\right)$, the amplification was conducted. The products followed an extension for 3 minutes at $72^{\circ} \mathrm{C}$, and were indefinitely held at $4{ }^{\circ} \mathrm{C}$. The PCR products were then separated on $2 \%$ agarose gel for 45 min at 120V, stained with GelRed (Crisafuli et al. 2015), and viewed by UV illumination.

Two positive controls (one male and one female) were used to assess the validity of the PCR amplification process, which were collected from fresh-frozen human tissue samples. Male control (Ctrl M) yielded two bands (163bp and 137bp), while female control (Ctrl F) yielded one band only (163bp). Five healthy (Figure 4) and five early OA subjects that showed female-specific bands were selected for further determination of 
their biological ages. The negative control (NC) displayed no signal, and thus consisted only of a master mix and no DNA contamination DNA. Moreover, no miss-amplification was observed and the implemented method was highly specific.

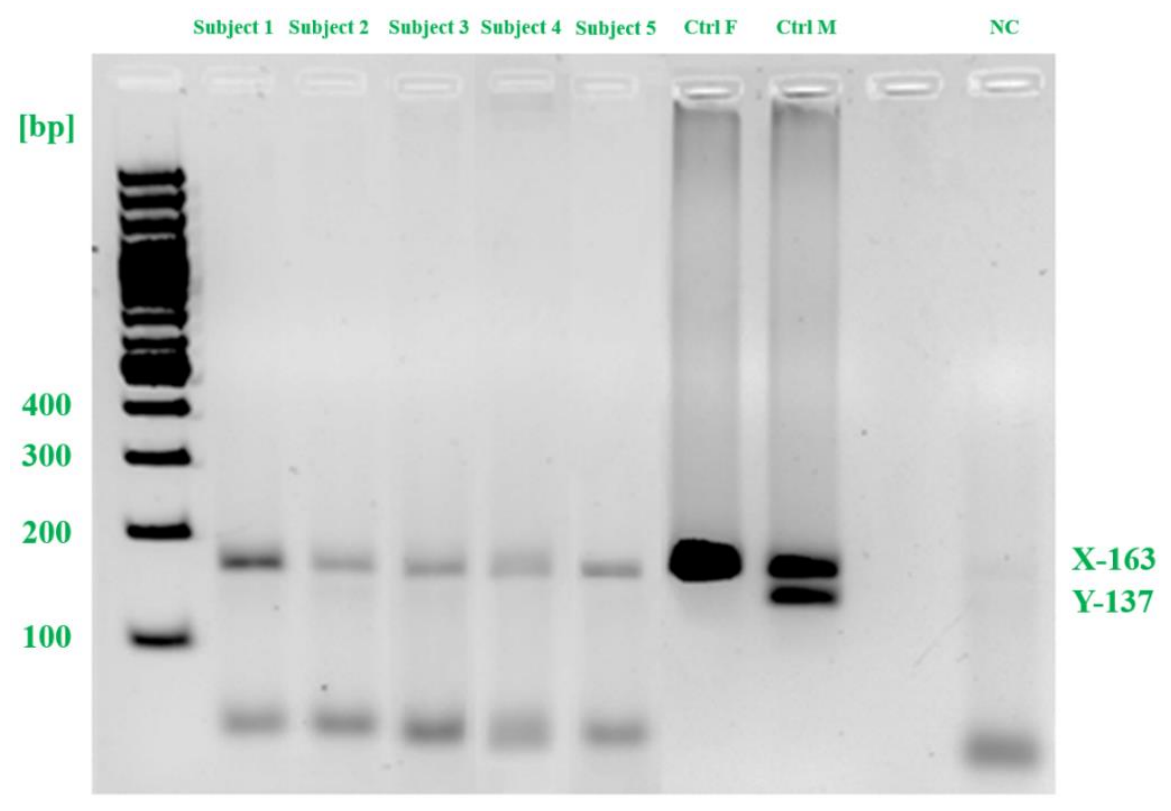

Figure 4. The PCR-based sex determination of the healthy human femurs. Marker shows the 100-bp ladder marker. The results of the sex identification of the subjects are illustrated in the lanes 2-6, while the lanes 7 and 8 represent the positive female $(\mathrm{Ctrl} F)$, and male controls $(\mathrm{Ctrl} \mathrm{M})$, respectively. The negative control (NC) consists only of master mix and no DNA contamination (lane 10).

\subsubsection{Determination of the biological age}

By analyzing the structural elements of the cortical bone, such as primary and secondary osteons, haversian canals, endosteal and periosteal lamellar bone, and bone resorption zones, whose quantity or distribution goes through age-dependent adjustments, it is possible to assess the biological ages of the subjects at death either quantitatively by use of a regression formula (Kerley and Ubelaker 1978; Andrea Drusini 1987), or by qualitative examination of the aforementioned structural elements. The precision of the quantitative method is declined if the examined bone surface shows degradation or is altered due to exposure to physical stress in different areas of the investigated crosssection (Andrea Drusini 1987). In that case, various areas of the bone's cross-section must be quantitatively assessed, making it a time-consuming method. Hence, a microscopybased qualitative technique was applied that evaluated the microstructure of the cortical bone. Different manifestations of the structural elements in different age-groups are seen in Figure 5 (Grosskopf 2004), and can be categorized into adult (20-40 years), mature (40-60 years), and senile (60+ years), while the adult and mature age-groups can be 
classified into three sub-groups (early, middle, and late) to further specify the biological age.
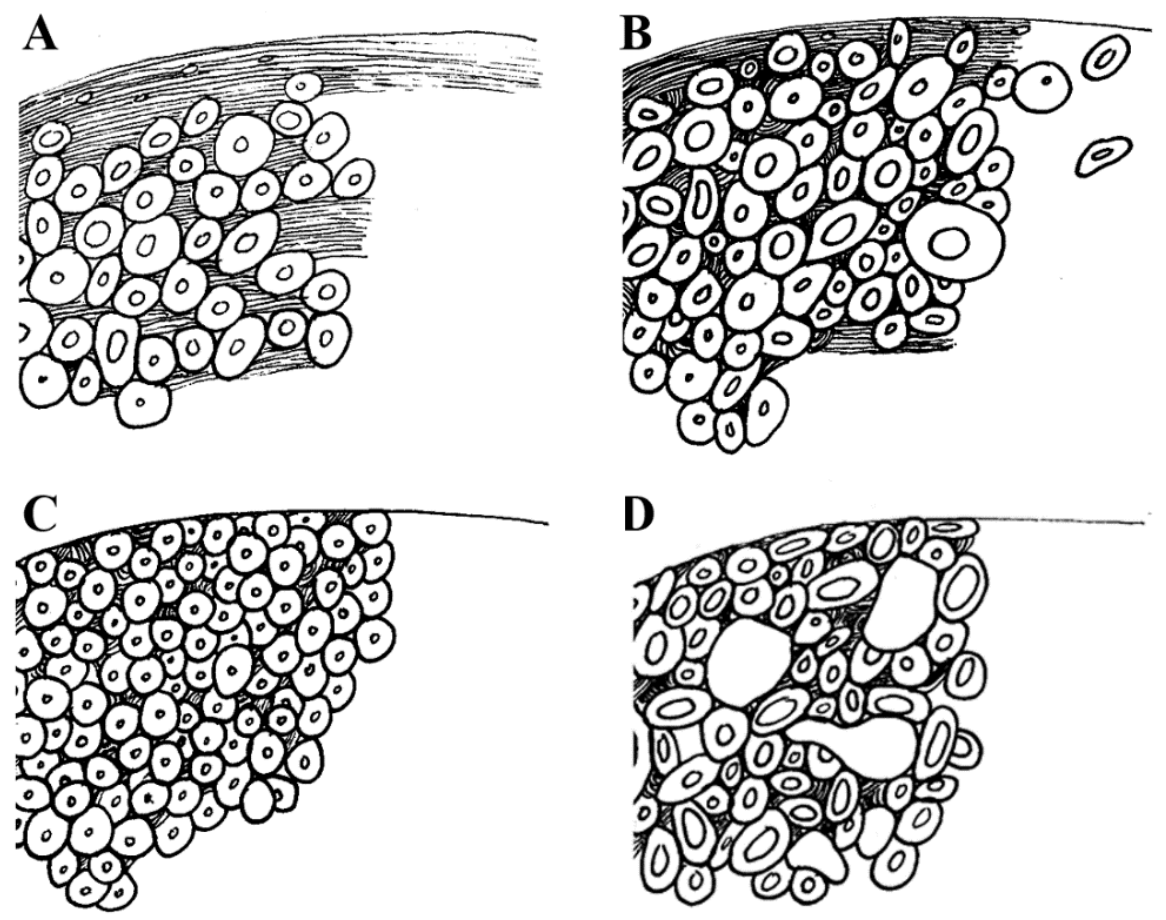

Figure 5. Schematic illustration of the cortical bone's microstructure in various age-groups. (A) juvenile, (B) adult, (C) mature, and (D) senile (Grosskopf 2004). "Grosskopf, 2004, used with the kind permission of Dr. Birgit Grosskopf"

Cortical bone extracts from the midshaft of the anterior part of the femur diaphysis were severed off from subjects 1-10. The bones were temporarily macerated according to the following protocol: Rinsed in an ultrasonic bath filled with distilled water for one hour to remove the surplus formalin solution while the distilled water was refreshed every 15 min. In a $10 \% \mathrm{NaCl} /$ detergent solution, bone samples were incubated overnight to remove fatty tissues. The periosteum was similarly removed by renewing and cooking of the solution for $4 \mathrm{~h}$. The cortical bone extracts were then relocated to a new $\mathrm{NaCl} /$ detergent solution and incubated at $50^{\circ} \mathrm{C}$ for 36 hours. They were rinsed once more in an ultrasonic bath filled with distilled water for $3 \mathrm{~h}$, while the distilled water was renewed every 30 min. In an ascending concentration of ethanol (i.e. 50\%, 70\%, 80\%, 96\%, 99.9\% abs.), the specimens were dehydrated (15 minutes for each sequence), transferred to acetone, and dried at $50^{\circ} \mathrm{C}$ overnight. Under vacuum condition, each sample was embedded in the epoxy resin Biodur ${ }^{\circledR}$ (Hagens 1979). In order to minimize optical artefacts during microscopic investigations, the resin was allowed to slowly impregnate the very fine canaliculi of the cortical bone (Grosskopf 2004). After approx. 72 hours, the epoxy resin 
was polymerized, and $100-\mu \mathrm{m}$ cross-sections were sawed using a microtome (Leica SP 1600, Leica Camera AG, Wetzlar, Germany). Finally, the microstructure of the bones was microscopically assessed with plain polarized light in various magnifications.

For the healthy femurs, it was possible to determine the biological age of four subjects at a sub-group precision (Table 4). They all had biological ages of 40 to 60 years at death, with the exception of one case, for which the age could not be more accurately classified than its general category (older than subadult). The microscopic picture of one cortical cross-section (subject 5) is shown in Figure 6, which revealed typical structural elements for early mature age-groups. For the early OA samples, aside from one sample that exhibited structural elements corresponding to the senile age-group, all subjects were either middle mature (mid-40 to 60 years) or late mature (mid-50 to 60; Table 4), and thus, were comparable to the cohort of the healthy samples (Subjects 1-5).

Table 4. Biological age determination of the healthy and early OA femurs

\begin{tabular}{|c|c|c|c|}
\hline Investigated bone & Sex & OA status & $\begin{array}{c}\text { Determined biological } \\
\text { age }\end{array}$ \\
\hline Subject 1 & female & healthy & $\begin{array}{l}\text { early mature (40 to } \\
\text { mid-40) }\end{array}$ \\
\hline Subject 2 & female & healthy & $\begin{array}{l}\text { late mature (mid-50 to } \\
60 \text { ) }\end{array}$ \\
\hline Subject 3 & female & healthy & $\begin{array}{l}\text { early mature ( } 40 \text { to } \\
\text { mid-40) }\end{array}$ \\
\hline Subject 4 & female & healthy & older than subadult \\
\hline Subject 5 & female & healthy & $\begin{array}{l}\text { early mature (40 to } \\
\text { mid-40) }\end{array}$ \\
\hline Subject 6 & female & Early OA & $\begin{array}{l}\text { late mature (mid-50 to } \\
60 \text { ) }\end{array}$ \\
\hline Subject 7 & female & Early OA & $\begin{array}{c}\text { late mature (mid-50 to } \\
60 \text { ) }\end{array}$ \\
\hline Subject 8 & female & Early OA & senile $(60+)$ \\
\hline Subject 9 & female & Early OA & $\begin{array}{l}\text { middle to late mature } \\
\text { (mid-40 to 60) }\end{array}$ \\
\hline Subject 10 & female & Early OA & $\begin{array}{l}\text { middle to late mature } \\
\text { (mid-40 to 60) }\end{array}$ \\
\hline
\end{tabular}

Determination of the biological age described in this section was developed in cooperation with the Johann-Friedrich-Blumenbach-Institute for Zoology \& Anthropology at University of Göttingen, and conducted by Dr. Birgit Grosskopf and Anna Lena Flux. 


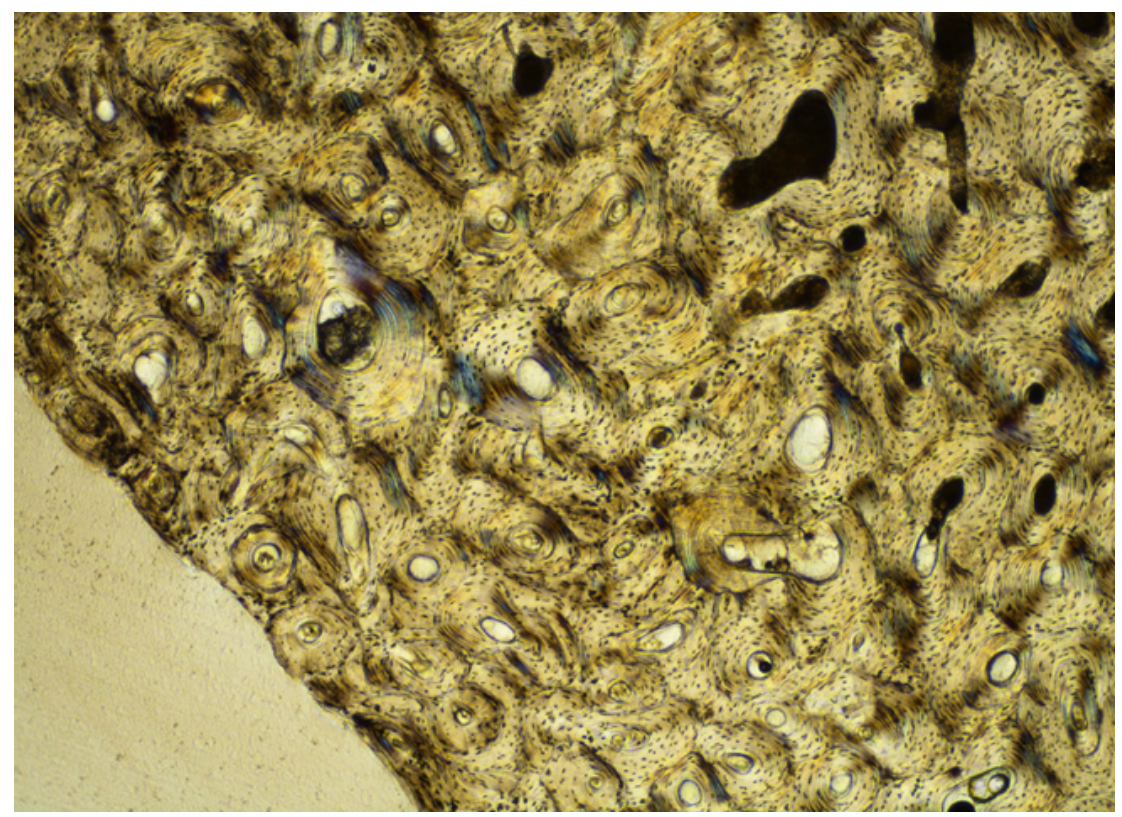

Figure 6. An exemplary microscopic image of the cortical bone's microstructure. The structure of the Subject 5 (40x magnification) is characterized by homogeneity at the outer general lamella on the periosteal side. At such areas, the shape and size of the osteons are uniform and the packing density is high. Remnants of the outer general lamella can only be observed sporadically; a hallmark of early mature individuals. At further distances from the periosteal side, irregularly-formed osteons can be observed that almost entirely replace the periosteal lamellar bone. The bone resorption areas are limited, which suggests that the subject died in the early mature age group.

\subsubsection{Assigning of the measuring points and biopsy}

To be able to scan the subchondral bone at a sufficiently high spatial resolution, the specimen has to be close to the X-ray source, which limits the maximum achievable voxel size. Considering that the smallest type of microchannels are reportedly in the range of 10-45 $\mu \mathrm{m}$ range (Clark 1990; Milz S. and Putz 1994), a voxel sized of $1.2 \mu \mathrm{m}$ was selected for image acquisition. Hence, 43 measuring points were defined on the surface of the healthy femoral heads, and 2.00-mm cartilage-bone cylinders were extracted using a trephine burr. The measuring points were defined as the convergence points of two sets of geometric shapes that were outlined on the surface of the joint: The first set composed of twelve concentric lines, separated each by a $30^{\circ}$-interval, starting from the center of the femoral head and ending on the femoral neck junction. The second set consisted of four parasagittal planes splitting the arc between the center and the neck junction into four evenly-spaced parallel regions (Figure 7). The center was identified by the intersection of the long axis and the coronal plane on the surface of the femoral head. With the aid of a template framework, the position of each set of measuring points was standardized taking 
into consideration the normalized size of each joint. The assigning method described here was partially inspired by the work of Turmezei et al. (Turmezei et al. 2014).
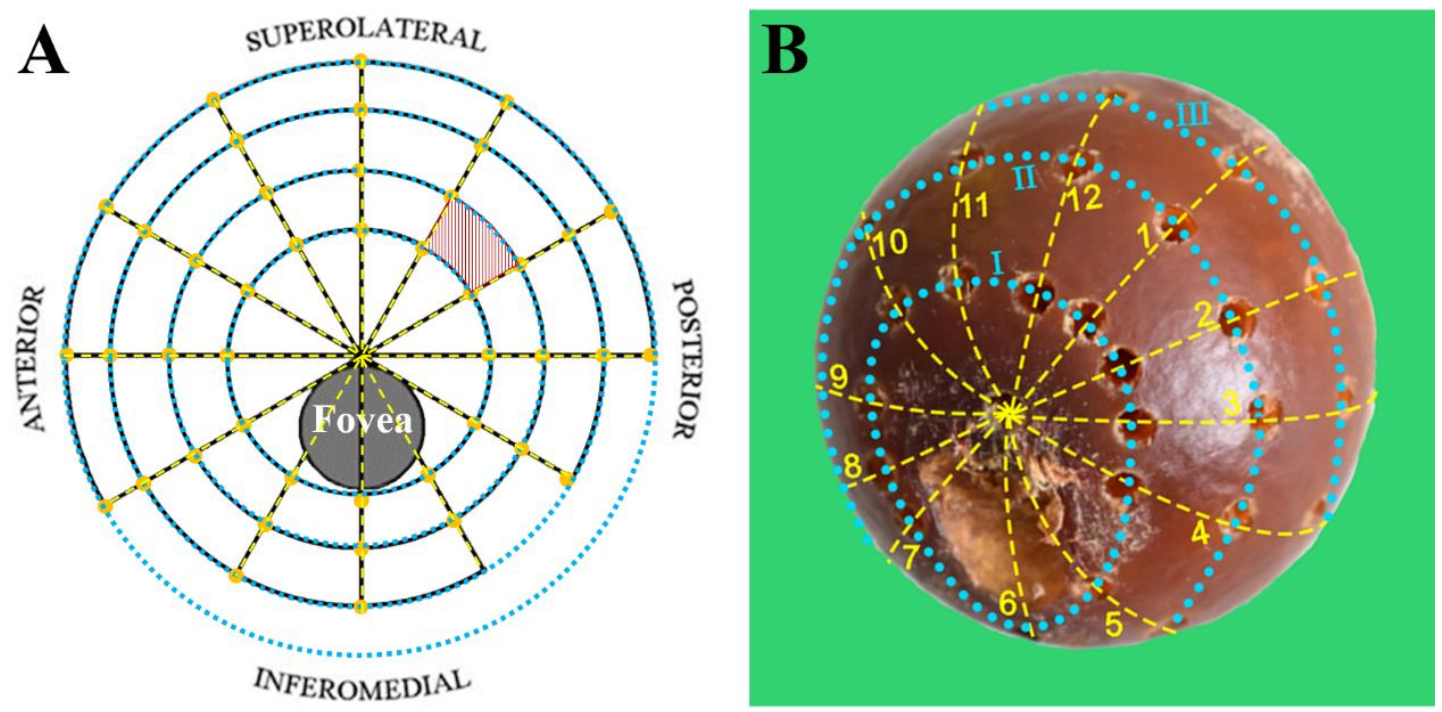

Figure 7. The system for assigning measuring points on the femoral head. (A) Measuring points are shown in orange as the intersections of twelve concentric lines (yellow dashed line), representing an analog clock model, and four parallel parasagittal planes (light blue, dotted circles). An exemplary sector is shown by crosshatched red lines. (B) The implemented model on a femoral head. twelve yellow concentric lines are numbered, while parasagittal planes are signified by Roman numerals.

For the early OA femurs, before drilling cartilage-bone biopsies, a 3D model of each subject was generated with the aid of a photogrammetry technique that comprised the whole epiphysis and metaphysis. Femurs were secured with a clamp, while the camera was moved in their surrounding 3D space at different relative heights while recording photos. The photos were imported into Agisoft PhotoScan (version 1.4.4, Agisoft LLC, St. Petersburg, Russia), and a sequence of analyses that included photo alignment, generation of dense could, mesh, and texture was applied to obtain the 3D models (Figure 8). The models were used as references for identifying local areas of partial-thickness defects, fibrillations, and fissures. The system based on the twelve concentric lines and the four parasagittal planes as previously described was used for defining the measuring points. These geometrical shapes divide each femoral head into 42 sectors, one of which is marked by red crosshatched lines in Figure 7A. The photogrammetry-generated 3D models were then superimposed on these sectors, while a maximum of four measuring points per sector was extracted in case early OA conditions as described in 2.1.2.1 were met. In total, 116 cartilage-bone biopsies were drilled out and scanned from OA-stricken 
areas. The SB microarchitecture in early osteoarthritis was then compared to that of healthy femurs in different loaded-areas on the joint.
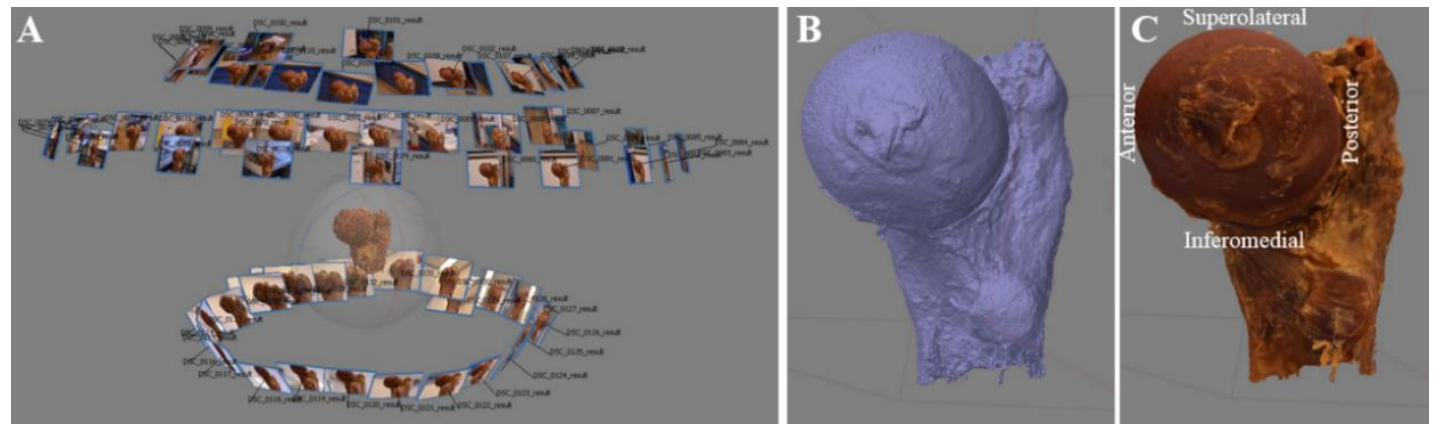

Figure 8. Workflow for 3D model generation of femurs by photogrammetry. (A) Photos were imported and aligned in three different relative heights to create sparse point clouds. (B) The point clouds were processed into a dense cloud, and the continuous mesh was subsequently produced over the surface of the model. (C) Finally, the original images were combined into a texture map and wrapped around the mesh, generating a photo-realistic 3D model of the object.

\subsubsection{Murine destabilization-induced osteoarthritis model}

\subsubsection{Animals}

Since destabilization of the medial meniscus (DMM) is known to be a testosterone-driven pathology (Ma et al. 2007), the experiment was conducted on male mice. 8 to 10-weeksold C57B1/6J wildtype (WT) mice were purchased (Charles River Laboratories, Sulzfeld, Germany) by the cooperation partners at the University of Regensburg, and were adapted until 12 weeks of age in standard conditions under a 12 hour cycle of dark/light sequences. For comparative analysis, age- and sex-matched substance P-deficient mice ( Tac1-/- , kindly donated by A. Zimmer, University Bonn, (Zimmer et al. 1998)) and aCGRP-/mice (generated by R.B. Emeson (Lu et al. 1999), kindly gifted from T. Schinke/M. Amling, Eppendorf University Hospital Hamburg) with wildtype backgrounds were used. Food and water were available for the mice ad libitum. All animal procedures and experiments were approved by the respective ethics committee (Regierung von Unterfranken, Bavaria, AZ 55.2-2531-2-289, date of approval July 27th 2016).

\subsubsection{Conduction of the medial meniscus destabilization}

The OA induction was performed by a technique specified by Glasson (Glasson et al. 2007), and in cooperation with the Department of Orthopaedic Surgery, Experimental Orthopaedics, Centre for Medical Biotechnology at University of Regensburg. Briefly, the mice were anesthetized intraperitoneally by fentanyl, medetomidine, and midazolam. 
By a three-millimeter surgical skin cut between the distal patella and the proximal tibia, the right knee joint was then exposed, while by a 1-2 millimeter medial notch to the patellar tendon, the articular capsule was incised. After careful visualization of the medial meniscotibial ligament by a micro scissor, the ligament was dissected in order to induce OA. The Sham surgery was conducted with only the visualization of the right knee ligament as the control group. Afterwards, the articular capsule, as well as the skin incision were closed, while analgesics were given to the animals (buprenorphine in $0.9 \%$ $\mathrm{NaCl}$ solution, $0.1 \mathrm{mg} / \mathrm{g}$ bodyweight). For recovery purposes, the animals were allowed to move unconstrained immediately after the surgery. 2- and 8-weeks post-operative, the mice were anaesthetized and sacrificed by cervical dislocation. Knee joints were fixed in $4 \%$ paraformaldehyde/PBS for $16 \mathrm{~h}$ and were either stored in $70 \%$ ethanol for micro-CT, or decalcified in $20 \%$ EDTA/PBS for histological analysis.

\subsection{Contact force mapping of the joint}

It was hypothesized that the characteristics of the SB microstructure are affected by local forces on the joint. Hence, it was important to determine the load mapping on the femoral head based on contact force estimations. This was conducted with the aid of a compatible musculoskeletal model (Gait 2392) that integrated 92 musculotendon units and 23 degrees of freedom, an open-source biomechanical analysis software (OpenSim (Delp et al. 2007)), and experimental data obtained from eight able-bodied men (age: $25 \pm 3$ years, height: $188 \pm 3 \mathrm{~cm}$, weight: $86 \pm 8 \mathrm{~kg}$ ). The gait analysis protocol was approved by the local ethics committee at the University Medical Center Göttingen, Göttingen, Germany (application number: 14/6/17), and the participants signed the consent form before the experiment. They walked at a relaxed pace of their own choice and completed four trials. Full-body kinematics and ground-reaction forces were recorded during each trial by a motion capture system equipped with 12 infrared cameras (Bonita B12, Vicon MX, Vicon Motion Systems Ltd, UK) and retro-reflective markers. The markers were positioned in clusters of three over bony landmarks as well as shanks and thighs (Ferrari et al. 2008). The sampling of marker trajectories was performed at $200 \mathrm{~Hz}$. Two identical force plates (9287A, Kistler Group, $\mathrm{CH}$ ) were used to register ground reaction forces, each of which recorded forces at $1000 \mathrm{~Hz}$ on one foot. The time window for analysis started with the left toe off for each trial, and finished with the right heel contact. Using a low-pass filter at $6 \mathrm{~Hz}$, the previously-measured marker trajectories and the ground reaction forces were filtered. A distinctive musculoskeletal model for each participant was created using the 
marker trajectories from a separate static trial, and the OpenSim 's Scaling feature. A sequence of analyses that included various OpenSim functions such as the Inverse Kinematics, Residual Reduction Analysis, Computed Muscle Control, and Joint Reactions Analysis was then conducted on the scaled model. The output of the last function was the estimated reaction forces on the hip joint. The estimated contact force at the femoral was normalized to each participant's body weight. Accordingly, different loading regions of the femoral head were identified based on all the participants' superimposed forces.

The contact force mapping of the joint described here was conducted in cooperation with the Applied Rehabilitation Technology Lab (ART-Lab) of the Department of Trauma Surgery, Orthopaedic Surgery and Plastic Surgery, University Medical Center Göttingen by Dr. Takashi Yoshida and Robert H. Foerster.

\subsection{Micro-computed tomography (micro-CT)}

\subsubsection{Bovine model}

Prior to scanning, the outer surface of the cartilage-bone biopsies was sanded and polished to acquire $4^{3} \mathrm{~mm}^{3}$ cubes. The samples were rinsed with a $70 \%$ ethanol solution to wash off possible artifacts, were placed in sample holders, and were scanned using a highdefinition micro-CT device (micro-CT 35, SCANCO Medical AG, Wangen-Brüttisellen, Switzerland). 600 slices were obtained for each biopsy using the following settings: Source voltage of $70 \mathrm{kVp}$, intensity of $114 \mu \mathrm{A}$, integration time $=1000 \mathrm{~ms}$, and an isotropic voxel size of $3.5 \mu \mathrm{m}$. A $0.5 \mathrm{~mm}$ aluminum filter was used to reduce the polychromaticity of the beam and pre-compensate for expected beam hardening. Segmentation was carried out by setting the lower threshold at $461 \mathrm{mg} \mathrm{HA} / \mathrm{cm}^{3}$, upper threshold at $3000 \mathrm{mg} \mathrm{HA} / \mathrm{cm}^{3}$, and the Gaussian filter Sigma and Support at 0.8 and 1, respectively. To study the entire subchondral zone morphometrically, a volume of interest (VOI) of 2000 (length) $\mu \mathrm{m} \times 700$ (height) $\mu \mathrm{m} \times 2000$ (width) $\mu \mathrm{m}$ was placed in the center of each scan, until $100 \mu \mathrm{m}$ below the cortical end-plate. To measure the morphometric parameters of subchondral bone's individual layers (i.e. CC and SBP), a VOI of 700 (length) $\mu \mathrm{m} \times 100$ (height) $\mu \mathrm{m} \times 700 \mu \mathrm{m}$ (width) was selected. For the calcified cartilage, the VOI was placed $50 \mu \mathrm{m}$ below the tidemark (Figure 9). The subchondral bone plate was defined by the calculated mean value of the calcified cartilage thickness plus its two- 
fold standard deviation. Accordingly, a same-sized VOI $(700 \times 100 \times 700 \mu \mathrm{m})$ was set $300 \mu \mathrm{m}$ deep in the micro-CT scans of the calf specimens, and at $435 \mu \mathrm{m}$ of the cattle.

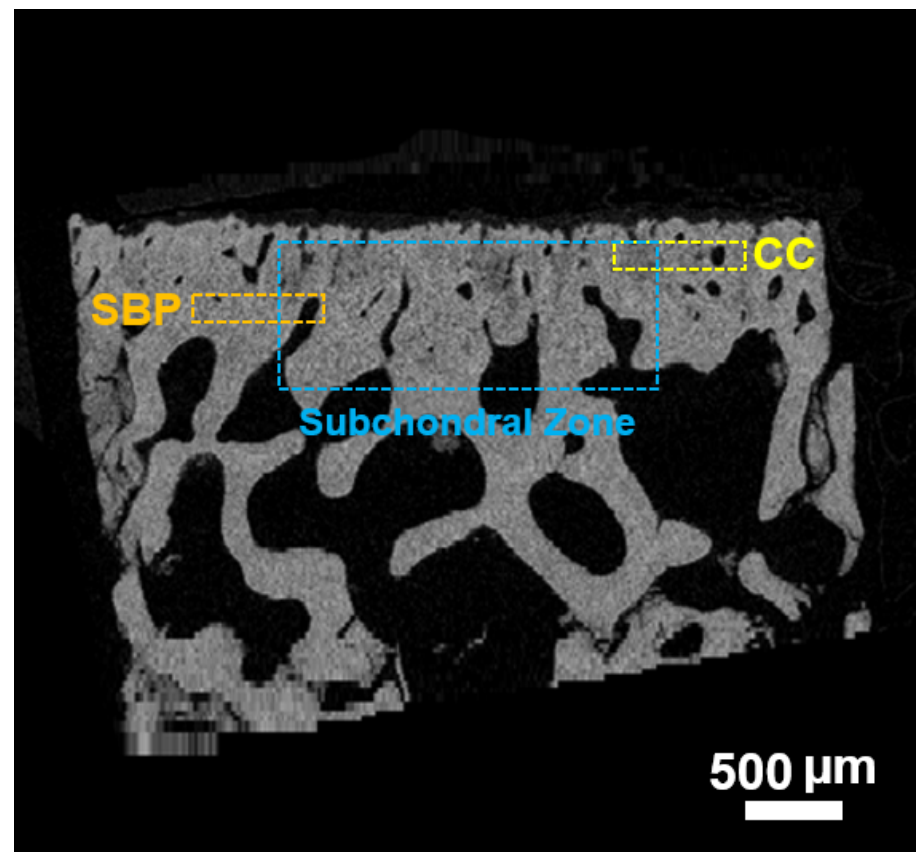

Figure 9. Volume of interests for measurement of different SB layers in calf. The VOI for calcified cartilage (CC) is placed $50 \mu \mathrm{m}$ below the tidemark, while it was set $300 \mu \mathrm{m}$ deep for the SBP. For the entire subchondral zone, it was placed until $100 \mu \mathrm{m}$ below the cortical end-plate, encompassing the uppermost portion of the trabecular bone.

The bone mineral density was measured using a grid containing $10 \times 8$ individual rectangles, where each row corresponded to a specific depth from the tidemark separated by $100 \mu \mathrm{m}$ intervals (Figure 10). In each rectangle, a measuring field of $6 \times 6$ pixels was places manually on the SB structure. In marginal areas where one pixel can be allocated to both bony and microporous structures, partial volume effect can occur, which may lead to an underestimation of the measurement values. To avoid this, care was taken to not position the measuring filed at the edges of bony structures. Moreover, very small bone cavities, under the resolution limit, can strongly alter the distribution of mineralization. The final mineralization value attributed to each depth was the mean \pm SD of the 10 rectangles for 3 samples per group. In order to minimize possible errors caused by the partial volume effect, measurements with a standard deviation of higher than $150 \mathrm{mg}$ $\mathrm{HA} / \mathrm{cm}^{3}$ were excluded.

To visually represent the $3 \mathrm{D}$ microchannels of the bovine $\mathrm{SB}$, the inverted $3 \mathrm{D}$ models were created by shifting the upper and the lower threshold values. By doing so, the 
visualization of cavity structures within the $\mathrm{SB}$ is made easier in a background of transparent bone tissue. Hence, the lower threshold was adjusted at $-500 \mathrm{mg} \mathrm{HA} / \mathrm{cm}^{3}$ while the upper threshold at $520 \mathrm{mg} \mathrm{HA} / \mathrm{cm}^{3}$ (Gauss filter unchanged).

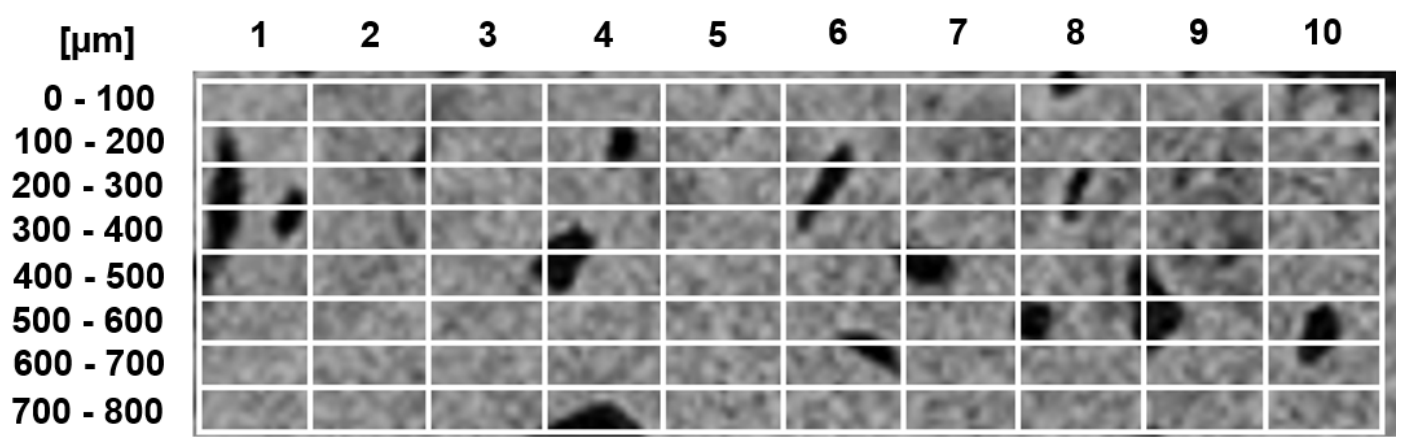

Figure 10. Measurement of bone mineralization in the bovine model. The values were measured in $100 \mu \mathrm{m}$ increments, until $800 \mu \mathrm{m}$ distance from the tidemark. For each $100-\mu \mathrm{m}$ region, the values were reported as the mean $\pm \mathrm{SD}$ of ten sequential rectangles.

\subsubsection{Healthy and early OA human femoral heads}

Before scanning, possible debris and artifacts around the cartilage-bone biopsies were washed off with $70 \%$ solution of ethanol. The biopsies were then positioned vertically into the sample holders and scanned using a compact cabinet micro-CT scanner ( $\mu$ CT 50, SCANCO Medical AG, Switzerland). Based on the smallest type of the SB channels reported in the literature (10-45 $\mu \mathrm{m})$ (Clark 1990; Milz S. and Putz 1994), a voxel size of $1.2 \mu \mathrm{m}$ was selected for the high-resolution scanning and further quantitative analysis of the SB microarchitecture. Other scanning settings included: Source voltage $=90 \mathrm{kVp}$, intensity $=88 \mu \mathrm{A}$, integration time $=1500 \mathrm{~ms}$, projections $/ 180^{\circ}=1500$, and a $0.5 \mathrm{~mm}$ aluminum filter.

A second dry scan at a lower resolution (voxel size $=14.8 \mu \mathrm{m})$, but a much faster scanning time was conducted in order to detect the articular cartilage as a lesser-dense object with minimum shrinkage (Figure 11). The AC was detected and segmented from the air and bone, using the 3D segmentation script of the Scanco's OpenVMS software and the following threshold setting: air: -500 - -140 $\mathrm{mg} \mathrm{HA} / \mathrm{cm}^{3}$, cartilage: -140-600 $\mathrm{mg} \mathrm{HA} / \mathrm{cm}^{3}$, bone: $600-3000 \mathrm{mg} \mathrm{HA} / \mathrm{cm}^{3}$, Sigma: 2, Support: 4. To calculate the articular cartilage thickness and the SB thickness, the segmented models were imported to Fiji (National Institutes of Health, Maryland, USA (Schindelin et al. 2012). The measurements were performed for each sample at 60 fixed spots within three evenly-spaced cross-sections of the model to acquire a mean value. 


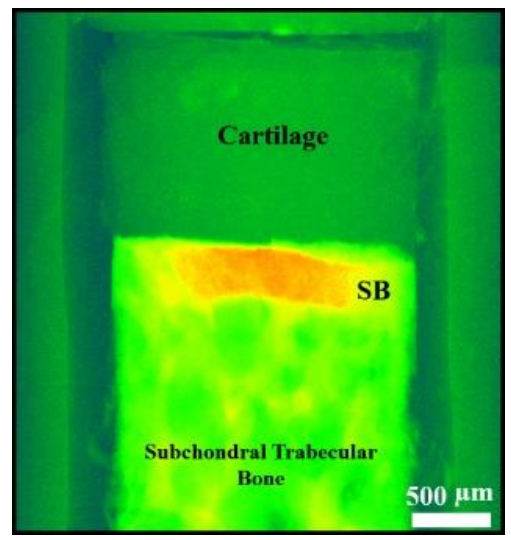

Figure 11. The scout view of a $2.00 \mathrm{~mm}$ cartilage-bone biopsy. The low-density articular cartilage, the high-density subchondral bone, and the subchondral trabecular region are marked.

In order to virtually cut the SB microchannels as perpendicular as possible, the uppermost surface of the subchondral bone must be consistently upwards. Hence, the lossless rotation command of the Scanco's image processing language was used to align the deviations that were more than one degree from the XY plane. After segmentation, binarized slices were generated as TIFF stacks, and were imported into Fiji for quantitative analysis (Figure 12). To prevent encircling possible bone debris and artifacts, the outermost $100-\mu \mathrm{m}$ rim of the samples were excluded. Thus, a cylinder with an $1800-$ $\mu \mathrm{m}$ diameter and a $400-\mu \mathrm{m}$ height was selected as the volume of interest, which spanned from the tidemark to the subchondral trabecular bone.
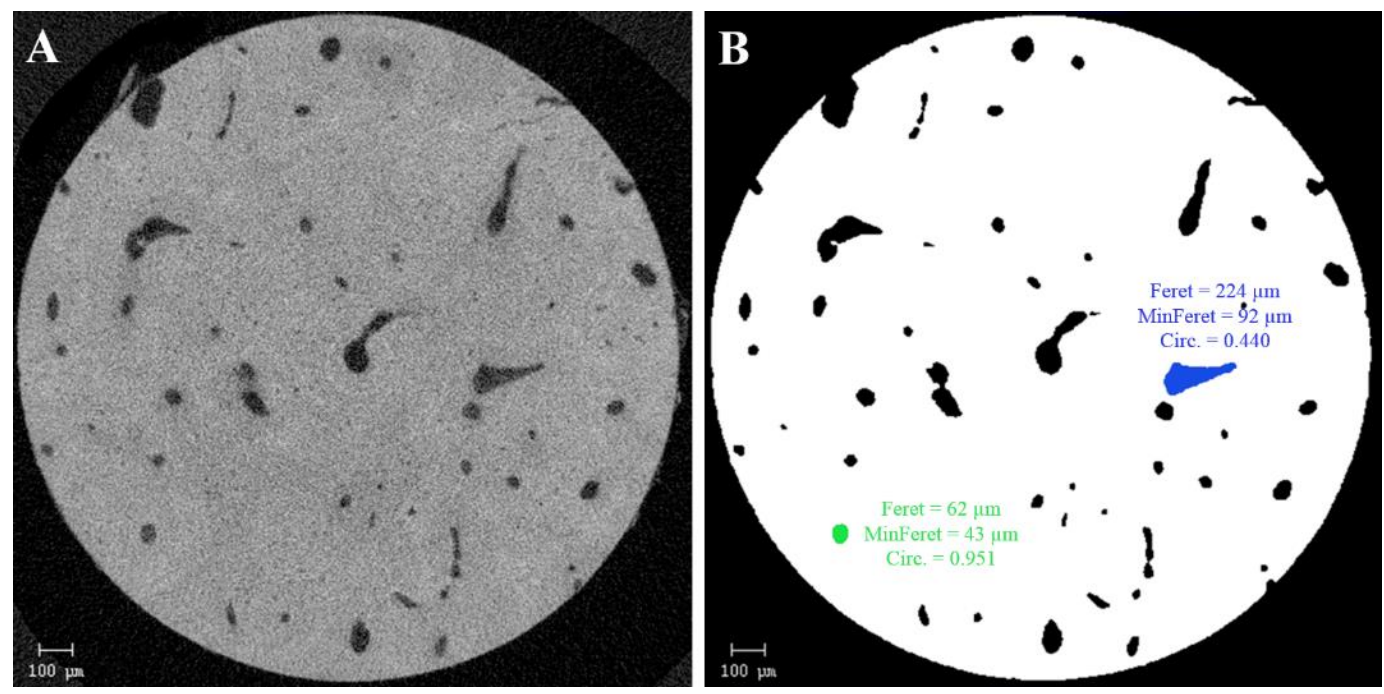

Figure 12. Segmentation of the SB microchannels. (A) The grey-scale image of the SB shows abundant perforations that reach to its superior surface. (B) The binarized image was then used for quantification. The quantified characteristics of two microchannels are provided as an example. 
The local density of the microchannels per $\mathrm{mm}^{2}$ was calculated as the "CMMC number". Since the cartilage-bone marrow microchannels were not necessarily circular, their size was quantified by measuring the maximum caliper diameter (Feret) and the minimum caliper diameter (MinFeret). They were defined as the longest distance between any two points along the CMMC, and the shortest distance within a microchannel's boundary, respectively. Likewise, the circularity was measured to provide a quantitative index for the morphology of the microchannels. For an arbitrary closed shape, it is defined as:

$$
\text { Circ. }=4 \pi\left(\frac{\text { Area }}{\text { Perimeter }^{2}}\right)
$$

A perfect circle has a Circ. of 1.0, while the value approaches 0.0 for increasingly elongated polygons. To quantify the CMMC's structural parameters on a layer-by-layer basis, a macro (Pseudocode 1) was developed that sequentially read the ROI of each slide, and calculated the CMMC's structural parameters with the "Analyze Particles" function. Intermittent pores below the $7 \mu \mathrm{m}$ Feret size were classified as surface craters and imperfections, associated with cartilage calcification (Clark 1990), and were filtered accordingly.

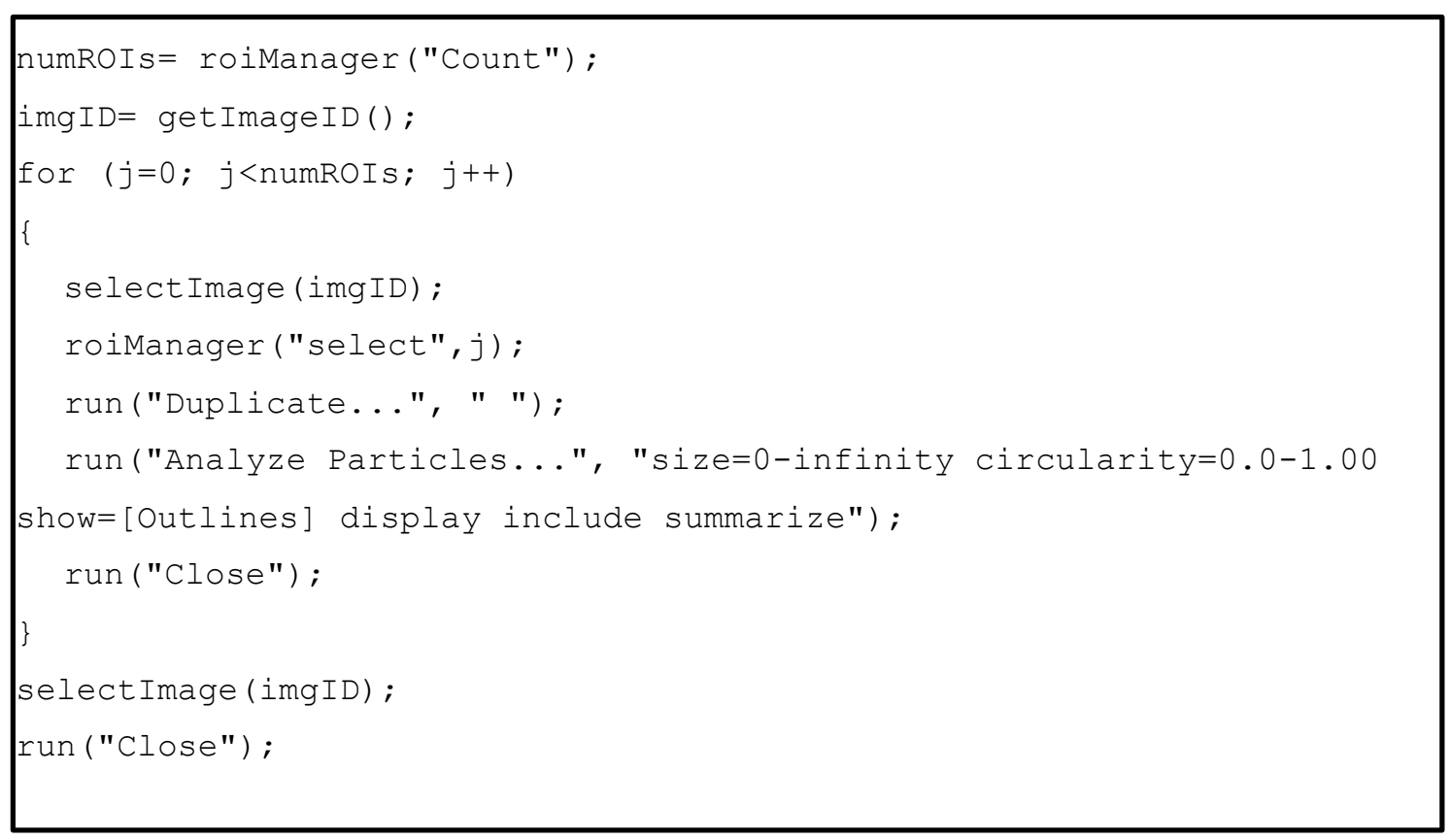

\section{Pseudocode 1}

Based on the contact force mapping of the joint and visual examinations of the subchondral bone microstructure, the micro-CT data were categorized and reported for three differently-loaded areas: i.e. load-bearing region (LBR), non-load-bearing region (NLBR), and the peripheral rim (PR). The changes of each structural parameter were illustrated as diagrams versus distance from the tidemark, where in each loading area, the 
profiles are means of the corresponding measuring points. At the articular cartilagesubchondral bone interface, the values are given as the mean \pm SD of the five subjects at the SB's uppermost $50 \mu \mathrm{m}$.

\subsubsection{Murine destabilization-induced osteoarthritis model}

Paraformaldehyde-fixed knee joints of wildtype, substance P-deficient, and aCGRP-/mice were prepared 2- and 8 weeks post-operative. They were scanned in $70 \%$ ethanol solution using a Scanco $\mu \mathrm{CT} 50$ device (Scanco Medical, Wangen-Brüttisellen, Switzerland; DFG number: 3230/30009760). To generate 3D models of the entire knee joint, image acquisition was performed at $6.8 \mu \mathrm{m}$ voxel size, $90 \mathrm{kVp}, 88 \mu \mathrm{A}, 800 \mathrm{~ms}$ integration time, and using a $0.50-\mathrm{mm}$ aluminum filter to reduce beam hardening. The 3D-reconstructed representations were then used to qualitatively examine the general topographical status of the joint, as well as potential osteophyte development and ectopic bone formations in meniscus. The quantitative analysis was based on a higher resolution scanning, which was conducted at $2.0 \mu \mathrm{m}$ voxel size $(1500 \mathrm{~ms}, 90 \mathrm{kVp}, 88 \mu \mathrm{A})$ for each knee joint. The segmentation was realized at recommended settings in accordance with the guidelines of Scanco (lower threshold: $685.3 \mathrm{mg} \mathrm{HA} / \mathrm{cm} 3$, upper threshold: $3000 \mathrm{mg}$ HA/ $\mathrm{cm} 3$, Sigma: 0.8, Support 1). For measuring the length of the medial and lateral condyles, the images were re-orientated by the ImageJ software (version $1.51 \mathrm{~J} 8, \mathrm{NIH}$, Bethesda, MD, USA), and measured at the $300 \mu \mathrm{m}$ distal depth from the subchondral bone's uppermost surface. The condyle lengths were regarded as the extent between the intercondylar tubercles in adjacency of the trochlear groove, and the medial or lateral prominence. The BMD and the bone volume fraction of the medial epiphysis was measured, the latter as an indicator of SB sclerosis. The VOI in the epiphysis had a 200 $\mu \mathrm{m}$ height, and started $270 \mu \mathrm{m}$ distally from the SB surface. Care was taken not to include the subchondral bone plate, the cortical bone, and the epiphyseal line. To quantify ectopic bone formation in meniscus after the DMM surgery, meniscal ossicles that located anteriorly were manually contoured and segmented with the aid of the Scanco's OpenVMS platform. Due to the post-operative erratic expansion of the ossicles' surface parallel to the longitudinal tibial axis, the height of the VOI varied for Sham- and DMMoperated mice, being $600 \mu \mathrm{m}$ and $1600 \mu \mathrm{m}$ for the Sham and the DMM mice, respectively. The bone volume (BV) and the BMD of the ossicles were measured using the Scanco's bone trabecular morphometry evaluation script. To extract the calcified cartilage layer from its underlying subchondral bone plate, a semi-automatic segmentation process was 
implemented, where an initial point cloud was created by setting the lower and upper thresholds values at 396.0 and $933.0 \mathrm{mg} \mathrm{HA} / \mathrm{cm}^{3}$, respectively. Nonetheless, since the grayscale values of the two adjacent layers are in the same vicinity, the segmentation provides a rough estimation of the layers, which can be enhanced by an interactive step based on a modified Seeded Region Growing (SRG) technique run by the OpenVMSbased script (Fan and Lee 2015). In the SRG, N seeds (pixels), which determine each region of interest, and are specified manually by the operator, can be collected in $\mathrm{N}$ sets $A_{1}, A_{2}, \ldots, A_{\mathrm{N}}$. $T$ is the set of all as-yet unallocated pixels which are adjacent to at least one of the pixels in $A_{\mathrm{i}}$ 's (Adams and Bischof 1994):

$$
T=\left\{x \notin \bigcup_{i=1}^{N} A_{i} \mid N(x) \cap \bigcup_{i=1}^{N} A_{i} \neq \phi\right\}
$$

Where $N(x)$ is the set of immediate neighbors of the pixel $x$. Each step of the algorithm involves the addition of one pixel, $x$, from $T$, to one of its neighboring regions, $A_{\mathrm{i}}$, if $N(\mathrm{x}) \cap A_{\mathrm{i}} \neq \varnothing$. This pixel is chosen according to the following homogeneity criterion: Assume $\mathrm{R}$ as the region of growing pixels as $\mathrm{R}=\left\{r_{1}, r_{2}, \ldots, r_{\mathrm{n}}\right\}$; i.e., there are $n$ pixels, with the greyscale value of each pixel as $g(x)$. Then, the pair of $(x ; R)$ is selected and added to the growing region such that the following criterion is minimized:

$$
\begin{gathered}
\delta\left(x, R, Y_{L}\right)=\frac{1}{(2 L+1)^{2}}\left\{\sum_{j, k=-L, \ldots, L ; j^{2}+k^{2} \neq 0}\left|g\left(y_{j k}\right)-\frac{1}{n} \sum_{i=1}^{n} g\left(r_{i}\right)\right|+\left|g(x)-\frac{1}{n} \sum_{i=1}^{n} g\left(r_{i}\right)\right|\right\} \\
=\frac{1}{(2 L+1)^{2}}\left\{\sum_{j, k=-L}^{L}\left|g\left(y_{j k}\right)-\frac{1}{n} \sum_{i=1}^{n} g\left(r_{i}\right)\right|\right\} \\
=\frac{1}{(2 L+1)^{2}}\left\{\sum_{j, k=-L}^{L}\left|g\left(y_{j k}\right)-M\right|\right\}
\end{gathered}
$$


where $M$ is the averaged grayscale value of the growing region, $R$. In other words, in the course of the growth process, when regulating which pixel $x$ should be incorporated to one of the growing regions, the modified SRG method takes the grayscale values of a set of neighboring pixels of $x$ into account. This set of neighboring pixels are defined as all the enclosed pixels by a square of size $(2 L+1) \times(2 L+1)$ centered at $x$; the choice of $L$ depends on the pixel size and is usually selected between 10 to 20 (Fan and Lee 2015). The calculated bins of values were used to create histograms, and subsequently colormaps of the $\mathrm{CC}$ thickness in each condyle were generated. Colormaps were scaled to a maximum value of $80 \mu \mathrm{m}$ for all samples.

\subsection{Histological methods}

\subsubsection{Mason-Goldner staining of the osteochondral junction in the bovine model}

To calculate the thickness of different SB layers in the bovine model, Mason-Goldner staining was used. First, undecalcified bone biopsies were fixed for one week in a solution of $4 \%$ neutral buffered formalin, were repeatedly washed, and dehydrated in ascending ethanol concentrations (70-100\%; each concentration for 3 days). They were subsequently embedded in methyl methacrylate (MMA; Technovit 9100 New, Heraeus Kulzer GmbH, Hanau, Germany); a monomer that polymerizes in relatively low temperatures (ca. $60^{\circ} \mathrm{C}$ ), penetrates readily into hard tissues, and can be dissolved with non-aggressive chemicals such as acetone (Erben 1997). $5 \mu \mathrm{m}$ slices were then cut and stained with the Masson Trichrome technique, after the MMA removal. Weigert's iron hematoxylin kit (Merck KGaA, Darmstadt, Germany) was used to stain the nuclei for 5 minutes. After several steps of washing, the samples were treated with an azophloxin solution for $10 \mathrm{~min}$. They were then rinsed in a bath of acetic acid (1\%), treated with phosphoric tungstic acid Orange $\mathrm{G}$ solution for 1 minute, followed by a second rinse in $1 \%$ acetic acid for another minute. Finally, a solution of light green SF was placed on the sections for $5 \mathrm{~min}$, and rinsed again in a $1 \%$ solution of acetic acid for five minutes (the used treatment and washing solutions were from the Masson-Goldner trichrome staining kit, Merck KGaA). Two histological slices from each sample were evaluated for the total number of six slices per group (calves and cattle). They were digitally photographed at a $4 \mathrm{x}$ magnification, and the thickness of the layers was measured by the ImageJ software (version 1.52a, NIH, Bethesda, MD, USA). The measurements were performed at 20 fixed spots in each image to calculate a mean \pm SD for each zone. 
The histological sections used in this section were developed and stained by Dr. Jozef Zustin in cooperation with the Institute for Pathology, University Medical Center Hamburg-Eppendorf.

\subsubsection{Staining of the subchondral bone in human femoral heads}

\subsubsection{Toluidine blue}

To validate the representation of the SB microchannels derived from the micro-CT imaging, toluidine blue staining was conducted. From differently-loaded areas on the femoral head, cartilage-bone biopsies were randomly selected and decalcified by

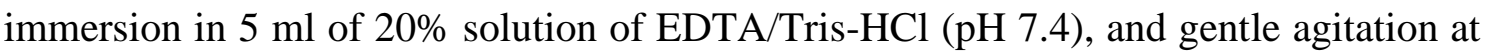
$4^{\circ} \mathrm{C}$ for $72 \mathrm{~h}$. After repeated washing with distilled water, they were dehydrated in ascending ethanol concentrations (70-100\%; each concentration for 3 days), were paraffin embedded, and then coronally sectioned in 5- $\mu \mathrm{m}$ slices. After removing the paraffin, the samples were then stained in $0.05 \%$ toluidine blue for $10 \mathrm{~min}$, dehydrated in ascending ethanol concentrations (70-100\%), and cleared in xylene.

\subsubsection{Hematoxylin and eosin (H\&E)}

The H\&E staining was performed according to routine protocols. Briefly, cartilage-bone biopsies were decalcified similar to 2.4.2.1, washed repeatedly, dehydrated by a graduated ethanol sequence, cleared in xylene, and processed in paraffin wax for embedding. 5- $\mu$ m-thick coronal sections were then cut through the sample by a microtome, and subsequently stained with $\mathrm{H} \& \mathrm{E}$ for routine histological examinations.

\subsubsection{3 von Willebrand Factor (vWF) immunostaining}

To perform vWF immunostaining, undecalcified cartilage-bone biopsies were prepared and embedded in MMA (Technovit 9100 New, Heraeus Kulzer GmbH, Hanau, Germany) similar to the descriptions in 2.4.1. For deplasticization, $5 \mu \mathrm{m}$ thick sections were placed in three changes of 2-methoxyethylacetate (Merck) for $10 \mathrm{~min}$ each, three changes of descending ethanol concentrations for $5 \mathrm{~min}$ each, and an eventual washing step in deionized water for $5 \mathrm{~min}$. The heat-induced antigen retrieval was conducted using a 0.01 M sodium citrate dihydrate solution $(\mathrm{pH} 6.0)$ at $60^{\circ} \mathrm{C}$ for $24 \mathrm{~h}$, with two PBS washing steps (each for $5 \mathrm{~min}$ ). Samples were further treated by $3 \%$ hydrogen peroxide solution (room temperature, $10 \mathrm{~min}$ ) with subsequent washing steps. Immunostaining was performed using anti-von Willebrand factor (A0082, Rabbit Polyclonal, Agilent 
Technologies, Santa Clara, US) at a 1:200 dilution (at $4^{\circ} \mathrm{C}$ for $24 \mathrm{~h}$ ) as the first antibody, EnVision+ System- HRP Labelled Polymer (Anti-Rabbit; Agilent Technologies, Santa Clara, US) as the secondary antibody (at room temperature for $1 \mathrm{~h}$ ), and the AEC enzyme substrate kit (for $30 \mathrm{~min}$; ab64252; Abcam, Cambridge, UK), with adequate washing and rinsing steps at each interval. The sections were finally counterstained with Mayer's hemalum solution (Merck, Darmstadt, Germany) for 5 min and mounted with an aqueous mounting medium (Aquatex; Merck, Darmstadt, Germany).

\subsubsection{Histology of the DMM murine model}

After fixation in $4 \%$ of paraformaldehyde/PBS solution for 16 hours, the knee joints were decalcified in 20\% EDTA/PBS (pH 7.4) for five weeks. After embedding in paraffin, 6 $\mu \mathrm{m}$ frontal slices were cut using a microtome. To visualize the status of cartilage integrity in different timepoints, five or six sections were deparaffinized for each sample at 60-90 $\mu \mathrm{m}$ intervals. They were subsequently deparaffinized, rehydrated and stained with Safranin O, Weigert's iron hematoxylin, and Fast Green, before scanning at 10x magnification by a TissueFAXS device (TissueGnostics, Vienna, Austria). The murine histological examinations were conducted in cooperation with the University of Regensburg.

\subsection{Statistical Analysis}

To assess the influence of age $\left(p_{\text {page }}\right)$ and distance from tidemark $\left(p_{\text {depth }}\right)$ on the morphometric indices of the bovine SB, one-way analysis of variance (ANOVA) was conducted. Gaussian distribution was ascertained by the Kolmogorov-Smirnov test and the homogeneity of variance was evaluated by Levene's test. The Bonferroni test was applied for comparisons in pairs where homogeneity of the variants was observed. The Dunnett T3 test was used as a post hoc analysis in case of inhomogeneity. Where the assumption of Gaussian distribution and homogeneity of variance was rejected, KruskalWallis one-way ANOVA was used, which included a pairwise post-hoc test. Friedman One-Way Repeated Measure Analysis of Variance by Ranks was carried out in order to test the hypothesis that differences in sample type (age) and areas of the subchondral bone (i.e. $\mathrm{CC}$ and SBP) results in different data distributions,

To determine the number of subjects for healthy and early osteoarthritis femoral heads, A power analysis was carried out using the $G^{*}$ Power software (Heinrich-Heine 
University Düsseldorf, (Faul et al. 2007)). A power of $80 \%$ and a standard deviation of $25 \%$ were assumed. Based on a pilot study, the effect size (Cohen's d) from two sets of estimated mean values were calculated: First, the CMMC number of the load-bearing region $\left(8\right.$ per $\left.\mathrm{mm}^{2}\right)$ and the non-load-bearing regions $\left(4\right.$ per $\left.\mathrm{mm}^{2}\right)$ of the femoral head. And second: the mean Feret sizes of the microchannels in the aforementioned regions (50 $\mu \mathrm{m}$ and $80 \mu \mathrm{m}$ in LBR and NLBR, respectively). Hence, 5 subjects were calculated with the Wilcoxon-Mann-Whitney test to ensure that means are different with an $\alpha \leq 0.05$. The scanned samples were regarded as paired to reckon with the multiple biopsies extracted from the same subjects. Therefore, different loaded regions were treated as the independent, within-subjects factor, and one-way analysis of variance (ANOVA) with repeated measures was performed for multifactorial comparisons of the variances in the group means. For early OA femoral heads, a Mixed-Design ANOVA was conducted with the health-state as the between-subject factor. The Gaussian distribution was tested by the Kolmogorov-Smirnov method, while the sphericity of the variables was evaluated by the Mauchly's test. For comparisons in pairs, the Bonferroni test was used as a post hoc test. In case of heterogeneity, the critical F values were calculated through the GreenhouseGeisser correction. To study potential correlations of AC thickness with other structural parameters of the SB, correlation analysis was conducted by Pearson test. Best linear fits were plotted, while the $p$ values were measured by a one-way ANOVA test.

For the murine destabilization-induced OA model, a similar power analysis was performed based on the OARSI score of paraffin sections as the key parameter. Hence, 6 animals per group were required for each group. Two-way ANOVA followed by a Tukey post-hoc test was conducted for the group comparisons of the tibial condyle length, CC thickness, as well as the BMD of the tibial epiphysis and the meniscal ossicles. For the micro-CT analysis of the murine DMM model, the results are reported as the mean \pm the standard error. All other results through this dissertation are given as mean \pm standard deviation. Statistical significance was set at alpha $=0.05$, while various levels of significance were denoted as: no significance (NS), $p<0.05(*), p<0.01(* *), p<0.001$ (***). All statistical evaluations were carried out using IBM SPSS Statistics (version 25.0, IBM SPSS, Armonk, NY, USA). 


\section{Results}

\subsection{Maturation-based changes of the SB in a bovine model}

The results presented in this section are based on the following published paper:

Taheri S*, Winkler T*, Schenk L*, Neuerburg C, Baumbach S, Zustin J, Lehmann W, Schilling AF (2019): Developmental Transformation and Reduction of Connective Cavities within the Subchondral Bone. Int J Mol Sci 20, 770

(* These authors contributed equally to this work and share the first authorship)

\subsubsection{Histomorphometry of the osteochondral junction}

The cartilage-bone biopsies that were drilled out from the medial condyle of the femurs (see 2.1) were assessed histomorphometrically. The SB was significantly thicker in cattle (mean $\pm \mathrm{SD}, 869.5 \pm 244.6 \mu \mathrm{m})$ than calves $(494.4 \pm 135.4 \mu \mathrm{m} ; p<0.01)$. Nonetheless, when individual layers of the SB were evaluated, it was found that the proportion of the calcified cartilage to the subchondral bone plate was relatively constant. The $\mathrm{CC}$ thickness of the calves, which appeared as a light-blue zone adjacent to the articular cartilage (Figure 13A) was $168.6 \mu \mathrm{m} \pm 63.7 \mu \mathrm{m}$, representing $34.1 \%$ of the entire SB thickness. In cattle, the CC thickness was significantly higher (265.2 $\pm 84.4 \mu \mathrm{m} ; \mathrm{p}<0.01$; Figure 13B), even though the ratio of CC/SB thickness was comparable to its calf counterpart $(30.5 \%)$. Similarly, the proportion of the subchondral bone plate to the entire subchondral bone was $69.5 \%$ and $66.3 \%$ for cattle and calves, respectively.
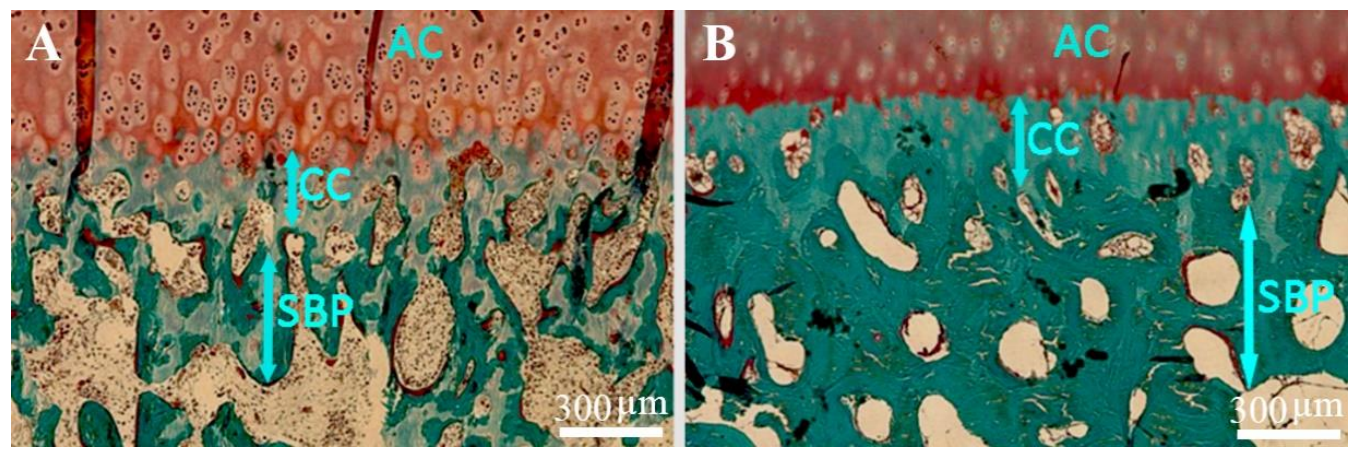

Figure 13. Masson-Goldner staining of the osteochondral junction. of (A) calves and (B) cattle. AC, CC, and SBP signify articular cartilage, calcified cartilage, and subchondral bone plate, respectively. 


\subsubsection{Micro-CT analysis}

\subsubsection{Analysis of the entire subchondral zone}

The microstructure of the entire subchondral zone, including the $100-\mu \mathrm{m}$ uppermost portion of the subchondral trabecular bone was analyzed, and the bone morphometry indices were summarized in Table 5.

Table 5. Micro-CT data of the entire subchondral zone in the bovine model

\begin{tabular}{cccc}
\hline Morphometrical indices & Calves & Cattles & Kruskal-Wallis \\
BV/TV [\%] & $48 \pm 4$ & $74 \pm 4$ & $p=0.049$ \\
Tb.Sp. $[\mu \mathrm{m}]$ & $45 \pm 6$ & $29 \pm 2$ & $p=0.046$ \\
Tb.N. [1/mm] & $11.6 \pm 0.8$ & $9 \pm 1.5$ & $p=0.049$ \\
Conn.D. [1/mm $]$ & $3835 \pm 1641$ & $1479 \pm 292$ & $p=0.049$ \\
DA & $1.14 \pm 0.04$ & $1.28 \pm 0.12$ & $p=0.275$ \\
Tb.Th. $[\mu \mathrm{m}]$ & $41 \pm 3$ & $85 \pm 20$ & $p=0.049$ \\
\hline
\end{tabular}

It was observed that the mean bone volume fraction (BV/TV; $<<0.05)$ as well as the thickness of the trabeculae (Tb.Th.; $\mathrm{p}<0.05$ ) significantly increased with maturation. On the other hand, the spacing between the trabeculae (Tb.Sp.; $p<0.05$ ), the number of trabeculae (Tb.N.; $p<0.05$ ), as well as the connectivity density (Conn.D.; $p<0.05$ ) showed maturation-related decline. For the whole subchondral zone, we observed no alteration in the degree of anisotropy. The reconstructed models showed that cattle had generally a denser bone structure with conspicuously thicker trabeculae in the subarticular region (Figure 14).
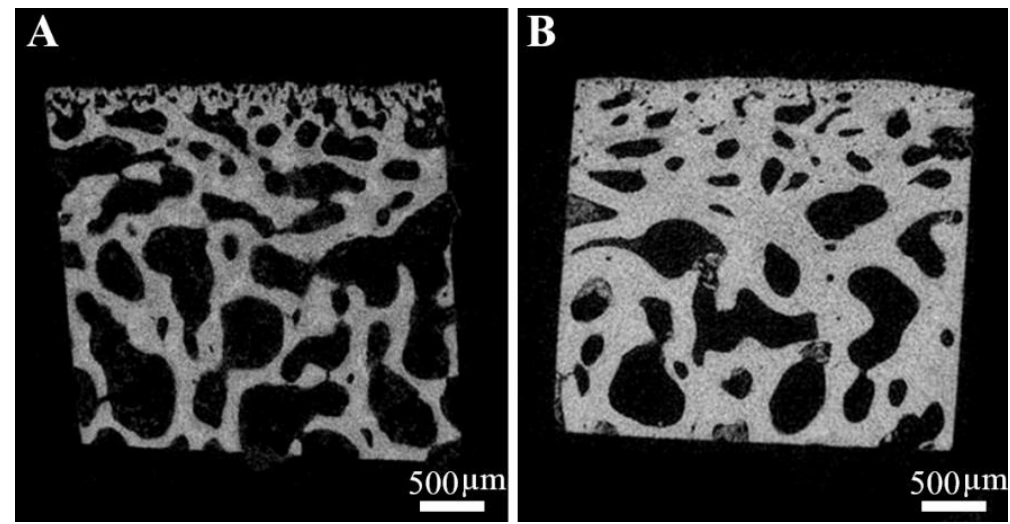

Figure 14. The image reconstruction of $\mathrm{SB}$ and subarticular region in bovine. (A) Calves revealed an intricate structure of thin trabecular bone, while (B) cattle was characterized by a compact bone structure and thicker trabeculae. 
3.1.2.2 Layer-by-layer analysis of the subchondral bone

By conducting the Friedman test, we tested the null hypothesis that the biopsies were of identical sources, and that age and region of the subchondral bone (i.e. CC and SBP) had no effect on the morphometric indices. The results are summarized in Table 6. The $p^{*}$ values for the Friedman test showed that almost all morphometric parameters were either age or region-dependent, thus, the null hypothesis was successfully rejected. A one-way ANOVA was then conducted to ascertain whether age or region was the deciding factor for each index. It was found that BV/TV, Tb.Sp., and DA were predominantly influenced by age (maturation), while the number and the connectivity of the trabeculae (Tb.N. and Conn.D.) were significantly affected by region of the subchondral bone. The BV/TV ( $p_{\text {age }}$ $=0.0002)$ and DA $\left(p_{\text {age }}<0.05\right)$ were significantly increased with maturation, while SB got denser with maturation as seen by the reduction of Tb.Sp. $\left(p_{\text {age }}<0.01\right)$ in cattle. On the other hand, the changes of the Tb.N. $\left(p_{\text {depth }}=0.001\right)$ and Conn.D. $\left(p_{\text {depth }}<0.05\right)$ were more sensitive to the region of the SB compared to the age, as they both significantly decreased from the calcified cartilage layer to the cortical end-plate.

Table 6. Layer-by-layer micro-CT data of the SB in the bovine model

\begin{tabular}{|c|c|c|c|c|c|c|c|}
\hline $\begin{array}{l}\text { Sample } \\
\text { Type (a) }\end{array}$ & Depth & $\begin{array}{l}\text { BV/TV } \\
{[\%] \text { Mean }} \\
\pm \text { SD }\end{array}$ & $\begin{array}{c}\text { Tb.Sp. }[\mu \\
\text { m] } \\
\text { Mean } \pm \\
\text { SD }\end{array}$ & $\begin{array}{c}\text { Tb.N.[1/m } \\
\mathrm{m}] \\
\text { Mean } \pm \mathrm{SD}\end{array}$ & $\begin{array}{c}\text { Conn.D } \\
{\left[1 / \mathrm{mm}^{3}\right]} \\
\text { Mean } \pm \\
\text { SD }\end{array}$ & $\begin{array}{c}\text { DA } \\
\text { Mean } \pm \\
\text { SD }\end{array}$ & $\begin{array}{c}\text { Tb.Th. }[\mu \mathrm{m} \\
\text { ] Mean } \pm \\
\text { SD }\end{array}$ \\
\hline Calf & $\mathrm{CC}$ & $56 \pm 4$ & $24 \pm 6$ & $18.3 \pm 2.2$ & $\begin{array}{c}5115 \pm 178 \\
3\end{array}$ & $1.32 \pm 0.05$ & $32 \pm 1$ \\
\hline Cattle & $\mathrm{CC}$ & $79 \pm 11$ & $10 \pm 6$ & $16.4 \pm 3.0$ & $3315 \pm 265$ & $2.17 \pm 0.85$ & $52 \pm 17$ \\
\hline Calf & SCBP & $48 \pm 3$ & $41 \pm 6$ & $12.1 \pm 0.9$ & $\begin{array}{c}1782 \pm 108 \\
5\end{array}$ & $2.01 \pm 0.12$ & $41.5 \pm 0$ \\
\hline $\begin{array}{c}\text { Cattle } \\
\text { Friedman' }\end{array}$ & SCBP & $77 \pm 11$ & $15 \pm 9$ & $12.7 \pm 0.5$ & $995 \pm 522$ & $3.05 \pm 0.6$ & $64 \pm 9$ \\
\hline $\begin{array}{c}\text { s } \\
\text { 2-way } \\
\text { ANOVA } \\
\text { by ranks }\end{array}$ & $p^{*}$ & $0.042 *$ & $0.042 *$ & $0.042 *$ & $0.029 *$ & $0.042 *$ & 0.072 \\
\hline \multirow{2}{*}{$\begin{array}{l}\text { One-Way } \\
\text { ANOVA }\end{array}$} & $p_{\text {age }}$ & $\begin{array}{c}0.0002 \\
* * *\end{array}$ & $0.0049 * *$ & 0.73 & 0.134 & $0.029 *$ & 0.006 \\
\hline & $p_{\text {depth }}$ & 0.634 & 0.15 & $0.001 * *$ & $0.027 *$ & 0.08 & 0.24 \\
\hline
\end{tabular}

\subsubsection{Mineralization}

The bone mineralization values were higher in cattle throughout the entire subchondral zone, as well as deeper subarticular region. The diagram of mineralization changes versus distance from the tidemark is presented in Figure 15. At the AC-SB interface (50-250 $\mu \mathrm{m})$ that represented the calcified cartilage layer, a steep slope was observed in the 
diagram. At further distances from the tidemark corresponding to the SBP and subarticular region, minimal depth-related changes were observed for the mineralization.

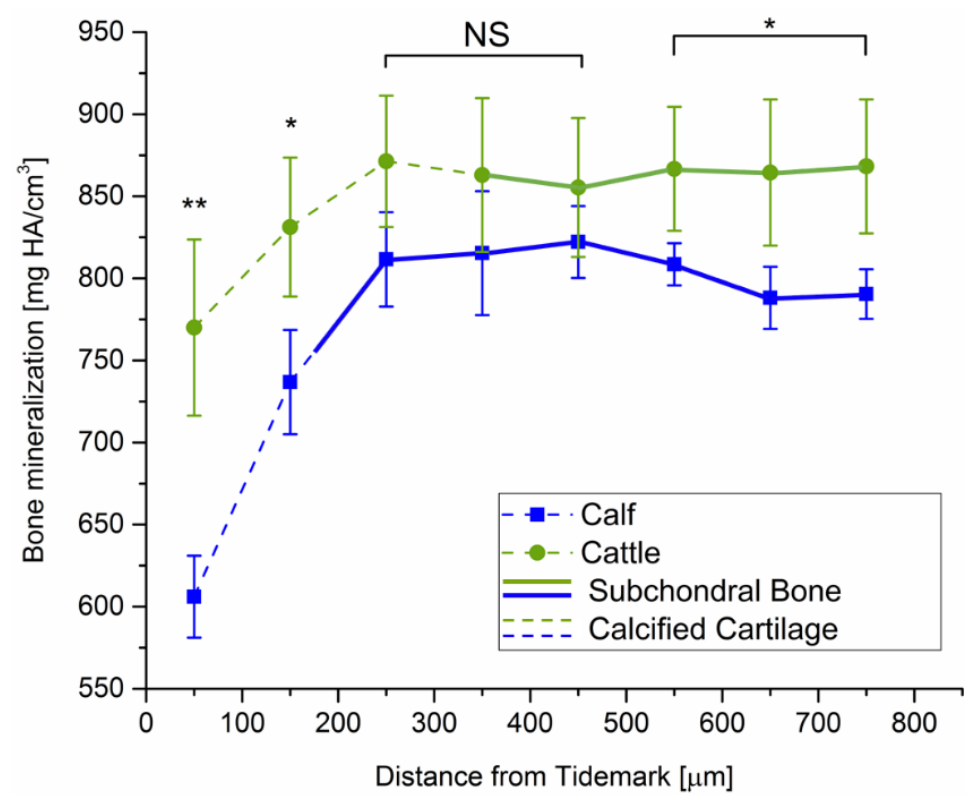

Figure 15. Profiles for the bone mineralization changes in the bovine model. At deeper distances from the tidemark, the BMD was markedly higher than the uppermost area of the SB for both age-groups. Compared to calves, cattle exhibited a consistently higher mineralization. This difference was significant close to the tidemark $(50-150 \mu \mathrm{m})$ and at the subarticular region $(550-750 \mu \mathrm{m})$, while insignificant at the midsection of the subchondral bone. $(* p<0.05$; ** $p<0.01)$

\subsubsection{Microchannels}

It was found that the microporous structure of the subchondral bone was not isolated and scattered but rather a continuous entity such that the lower margin of the articular cartilage was directly connected to the marrow cavity and subchondral trabecular bone through a multitude of microchannels. The 3D-reconstructed models showed that the uppermost surface of the subchondral bone was populated with perforations (Figure 16). 3D-models of the inverted images were created in order to present the spatial distribution of the microchannels with the SB. Hence, the blue-grey areas in Figure 16C,F represented cavity structures, while the transparent region signified bone matrix. Generally, the local density of the microchannels was higher in calves (Figure 16A), while fewer channels were found on the surface of the SB in immediate contiguity with the basal surface of the cartilage (Figure 16D). On the other hand, the SB microchannels were smaller in calf, and much wider in 18-month cattle. 

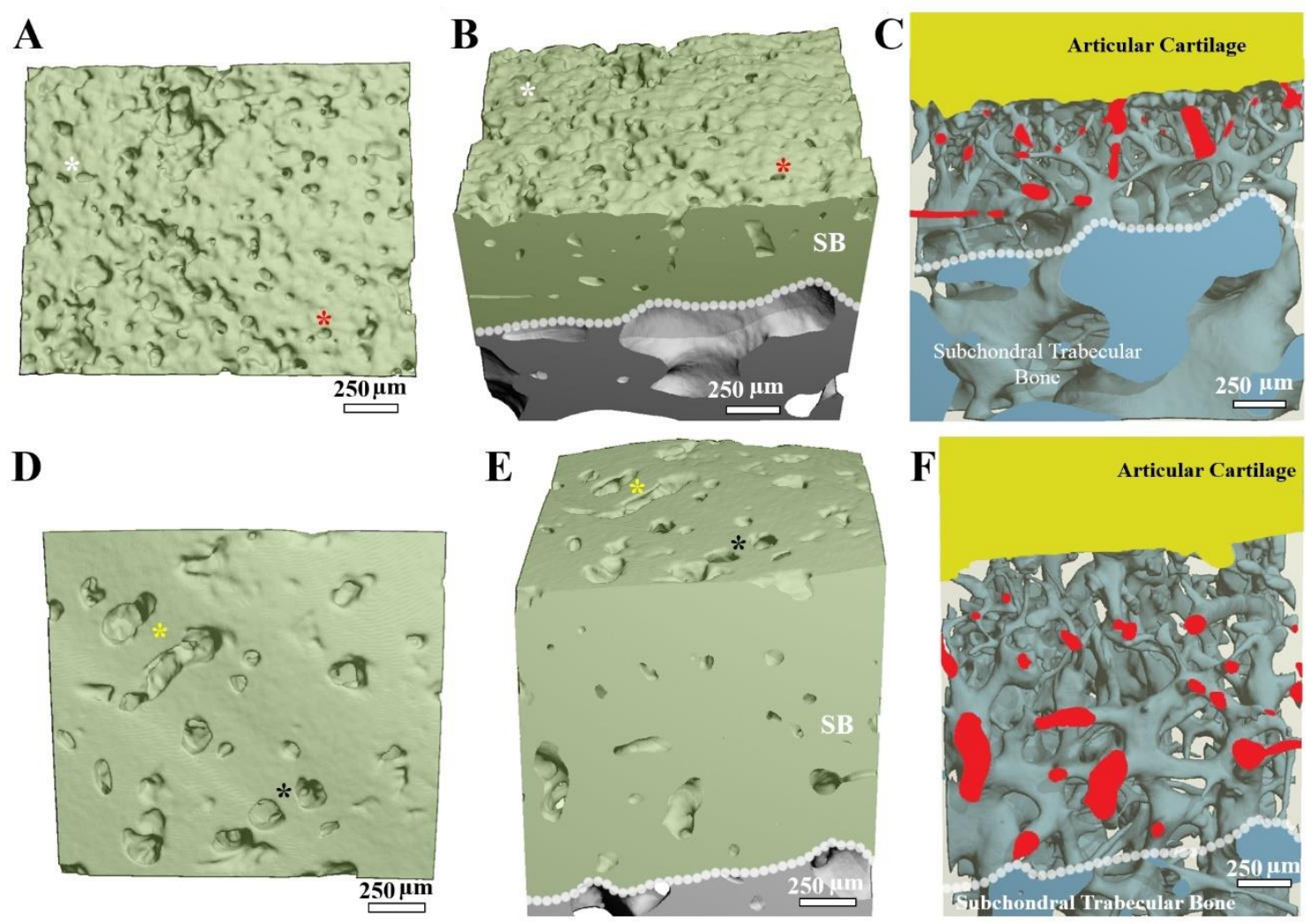

Figure 16. The 3D representation of the SB microchannels in the bovine model. (A) The superior view of the calf SB revealed plenty perforations at the uppermost surface of the SB. (B) The same volume of interest as shown in "A" was tilted slightly, where some of the microchannels were cut through the plane of sectioning. The asterisks on the SB surface are provided as reference points. The white dotted line represents the lowest border of the cortical end-plate. (C) In the inverted, negative model of the calf subchondral bone, the non-osseous structures were illustrated in blue-grey, while bone was transparent. The microchannels were visible throughout the whole thickness of the $\mathrm{SB}$, and provided a direct connective pathway between the subarticular region and the basal layer of the articular cartilage. Individual microchannels cut by the plane of sectioning are shown in red. In cattle (D) the perforations were less frequent on the superior surface of SB but much bigger in size. (E) The oblique view showed that SB was much thicker compared to calves, with sporadic perforations that were cut by the plane of sectioning. (F) The inverted model, however, showed the sporadic perforation (red) were in fact part of the continuous SB microchannel network. 


\subsection{Mapping of the SB microchannels in healthy human femurs}

The results presented in this section are based on the following submitted paper

Taheri S, Yoshida T, Böker KO, Foerster RH, Jochim L, Flux AL, Grosskopf B, Lehmann W, Schilling AF: The contact force of the human femoral head correlates with the microchannel architecture inside the subchondral bone. submitted to Calcified

\section{Tissue International}

\subsubsection{The superolateral area of the femoral head is the load-bearing region}

With the aid of a gait analysis, the amplitude and orientation of the hip contact force was estimated during self-paced walking. The contact force was continuously pointing the medial direction, had a broad superior portion, while peaking twice in the superoanteromedial direction during stance. Subsequently, the superimposed forces of all the participants were generated, and used as the basis to identify the load-bearing region (LBR) of the joint (Figure 17A). Out of the 43 measuring points that were defined on each femoral head, twelve were situated on the LBR. Likewise, the non-load-bearing region (NLBR) of the joint constituted another twelve measuring points but were mostly at the inferior half of the femoral head, and were located centrally (Figure 17B). The remaining 19 measuring points were situated at the peripheral rim (PR), corresponding to the two outermost parasagittal planes near the femoral neck junction.

A

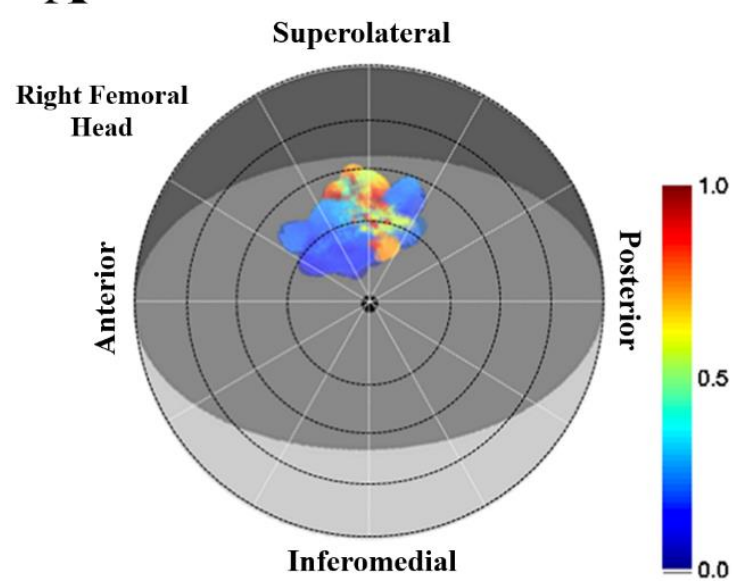

B

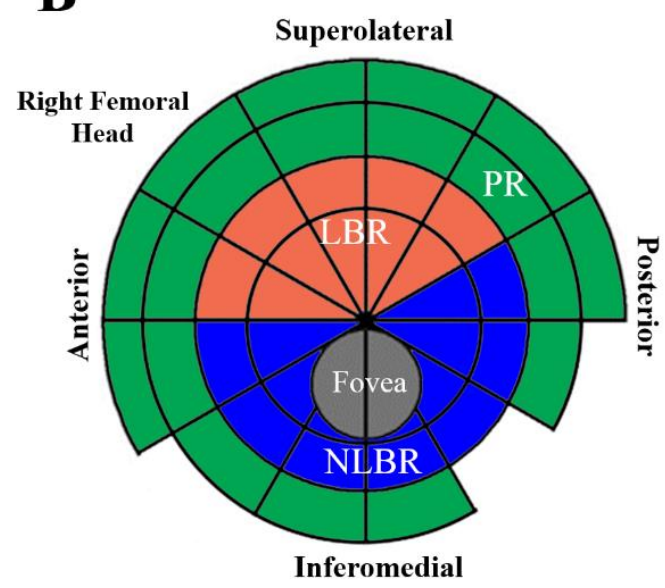

Figure 17. Contact force mapping of the femoral head. (A) The contact forces of all the participants in the gait analysis was superimposed and normalized to its local maximum within the time frame of analysis. It is viewed at azimuth of $-15^{\circ}$, and elevation of $50^{\circ}$ (B) Loading regions are then determined and colorcoded: load-bearing region (LBR; orange), non-load-bearing region (NLBR; blue), and the peripheral rim 
(PR; green). The gray circle in the close proximity to the NLBR is the fovea capitis. Due to the natural extension of the femoral neck close to the view, the inferomedial part of the rim is asymmetrical.

\subsubsection{The thickest area of cartilage and subchondral bone coincide}

By conducting a repeated measures ANOVA, a significant effect was observed in the AC thickness based on region (Wilks' Lambda $=0.027, \mathrm{~F}(2,8)=53.65, p=0.004, \eta^{2}=$ 0.973). Pairwise comparisons showed that articular cartilage was significantly thicker in the LBR (mean $\pm \mathrm{SD}, 809 \pm 119 \mu \mathrm{m})$ than the NLBR $(497 \pm 22 \mu \mathrm{m} ; p=0.00036)$ and the PR of the joint (293 $\pm 18 \mu \mathrm{m} ; \mathrm{p}<1 \mathrm{e}-5$; Figure 18A). A colocalization of cartilage and SB thicknesses was observed, where the SB was significantly thicker in the LBR $(241 \pm 42$ $\mu \mathrm{m})$ than the NLBR $(185 \pm 25 \mu \mathrm{m} ; p=0.02)$ and the PR $(154 \pm 40 \mu \mathrm{m} ; p=0.0015$; Figure 18B). A significant difference between the AC thickness of the NLBR and the PR ( $p=$ 0.0057) was detected, while the SB thickness was not statistically different between these two regions.
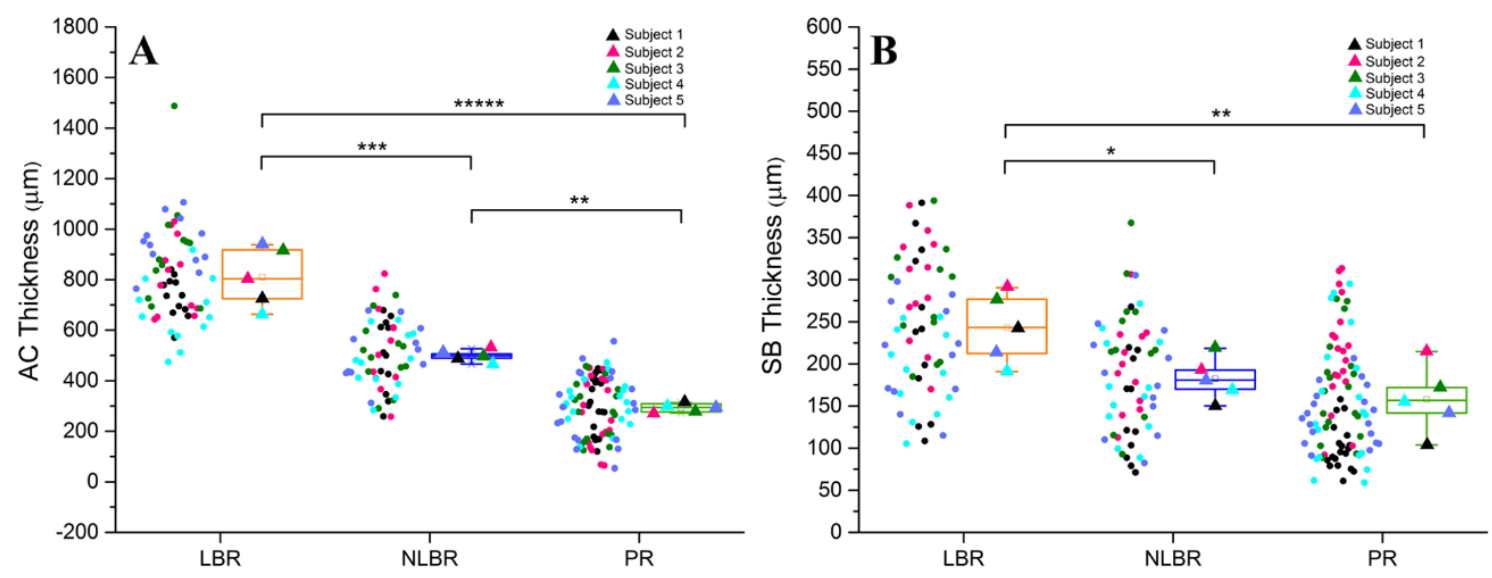

Figure 18. The changes of the AC and SB thicknesses in different loading areas of the femoral head. Circles near the boxplots represent the mean value of a measuring point, which are color-coded to their corresponding subjects. The triangles overlying the boxplots denote the mean values of corresponding subjects in each loading region. Repeated measures one-way ANOVA followed by Bonferroni post-hoc test. $* p<0.05 ; * * p<0.01 ; * * * p<0.001 ; * * * * *<<0.00001$

\subsubsection{Cartilage is connected to the marrow cavity via a complex microchannel network}

The subchondral bone's 3D-reconstructed models verified the existence of a continuous microporous structure that linked the $\mathrm{AC}-\mathrm{AB}$ interface directly to the marrow cavity, hence referred to as cartilage-bone marrow microchannel connectors (CMMC). It was observed that the CMMC had a distinct location-specific distribution pattern, where 
several narrow channels branched into the load-bearing region of the SB's uppermost surface and were in direct contact with the lowest margin of the AC (Figure 19A,B). The continuity of the microchannels throughout the entire SB was manifested by the inverted 3D-representation of the osteochondral junction (Figure 19C). Compared to the LBR, the microchannels were mostly distributed intermittently in the NLBR, and their sizes at the top surface of the SB were larger (Figure 19D-F). The largest channel sizes were observed at the peripheral rim of the femoral head, where the subchondral bone's microarchitecture consisted mainly of irregularly-shaped CMMC (Figure 19g - I).
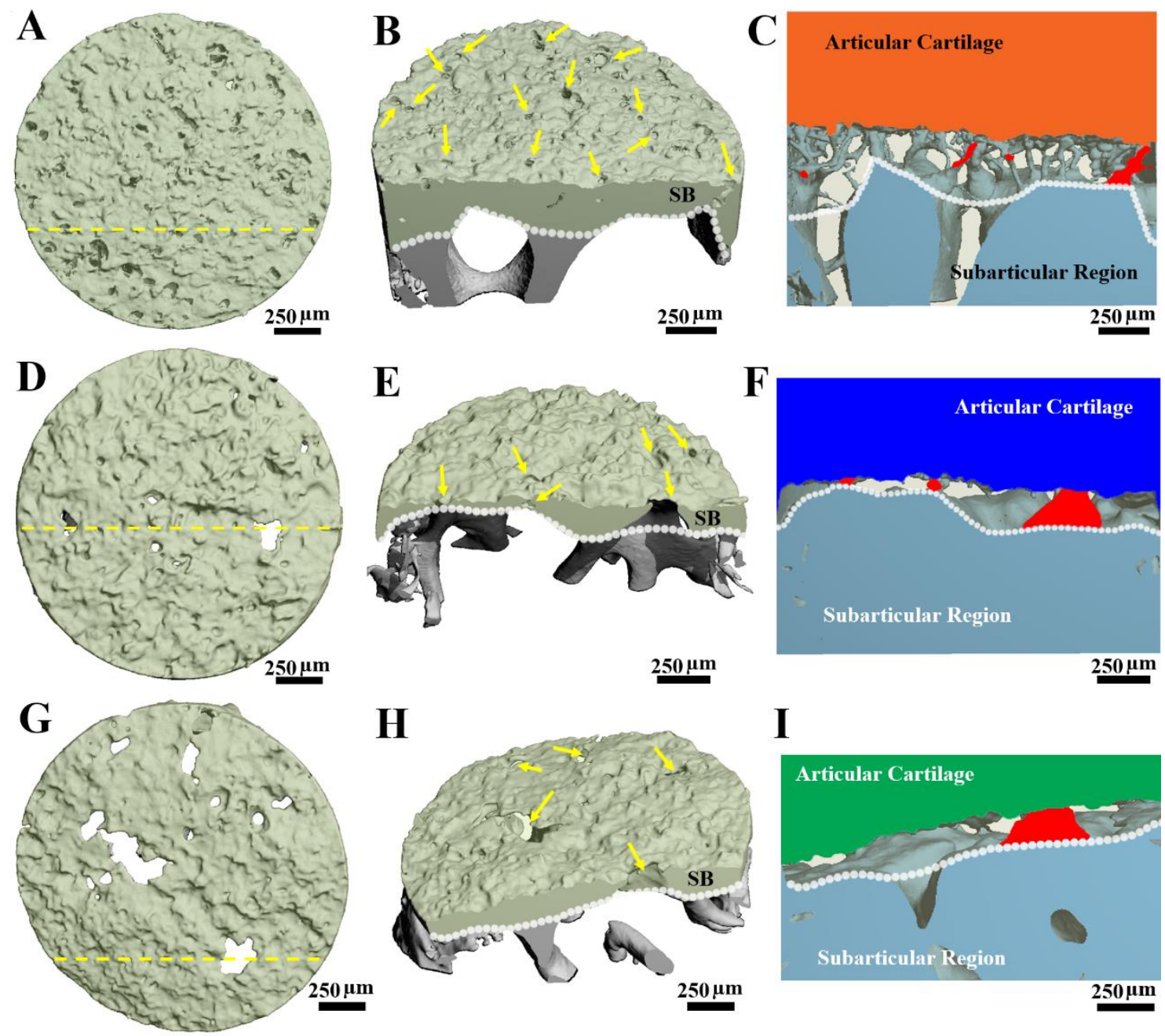

I

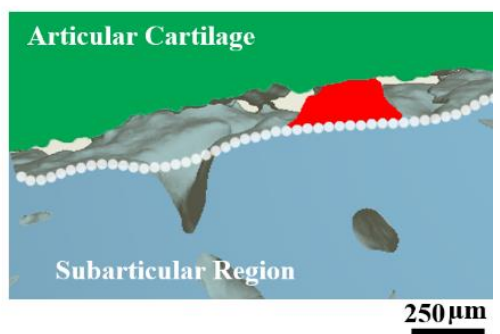

Figure 19. 3D representation of the SB microchannel network in healthy human femoral heads. (A) In a typical sample that was extracted from the LBR, several perforations were observed on the uppermost surface of the SB when viewed superiorly. The 3D model was virtually cut at an arbitrary plane of sectioning (the yellow dotted line) and viewed in (B), where connective pathways between AC and subarticular region were marked by yellow arrows. (C) In the inverted 3D model of the identical crosssection, load-bearing region cartilage was shown in orange, bony structures were transparent, and the CMMC was illustrated in blue-grey. The microchannels were visible throughout the whole thickness of the $\mathrm{SB}$, while individual microchannels cut by the plane of sectioning were marked in red. Similarly, the 
superior, coronal, and the inverted 3D-representations of typical samples from the NLBR and PR were presented in D-F and G-I, where articular cartilage was shown in blue (F) and green (I), respectively.

\subsubsection{Microchannels often appear in coronal histological sections as remote pores}

To obtain an understanding of the spatial configuration of the $\mathrm{CMMC}$, toluidine blue staining of the osteochondral junction was performed, and an exemplary NLBR slide was juxtaposed with the corresponding 3D-reconstrued model, as well as the inverted model, both derived from the micro-CT imaging (Figure 20). Sporadic pores within the subchondral bone of the 3D model were detected (marked by asterisks in Figure 20A), and the observation was confirmed in the equivalent histological slide. Nonetheless, the inverted 3D model revealed that these irregular, remote micropores were in fact crosssections of the SB's microchannel network (Figure 20C).
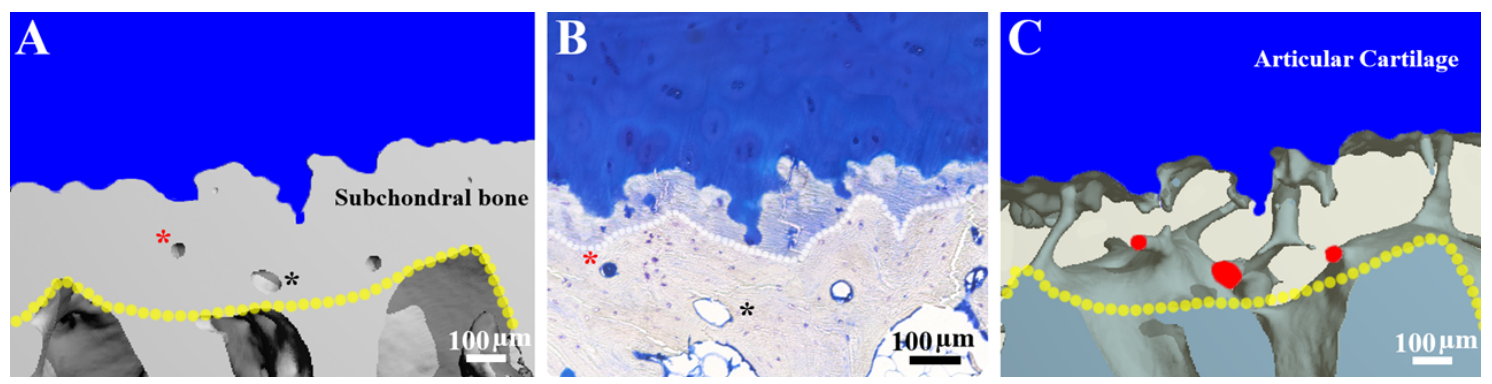

Figure 20. 3D imaging-histology juxtaposition of the osteochondral junction. (A) The 3D-reconstruced model on an exemplary NLBR sample revealed arbitrary micropores within the SB (marked by asterisks). The yellow dotted line signified the lowest margin of the cortical end-plate. (B) Similar geometrical microfeatures were observed by the toluidine blue staining, while the cement line was detected as the intersection between the calcified cartilage and the cortical end-plate (white dotted line). (C) the negative model showed that the so-called arbitrary micropores (marked in red) were part of the CMMC, and extended to the background of the subchondral bone.

Interestingly, further histological examinations showed that prolongations of the AC occasionally advanced through the calcified cartilage layer and were in touch with the cortical end-plate (Figure 21A). Moreover, in two sequential slides of an LBR-sample (Figure 21A, B), the evolution of a representative dendric channel was depicted, again highlighting the complex three-dimensional nature of these microfeatures. Directly at the lowest margin of the $\mathrm{AC}$, the $\mathrm{CMMC}$ either circumvented the $\mathrm{CC}$ layer to abut the $\mathrm{AC}$ or forged through it (Figure 21C). The cement line appeared as a particularly irregular margin that separated the CC from the SBP (white dotted line in Figure 21). 

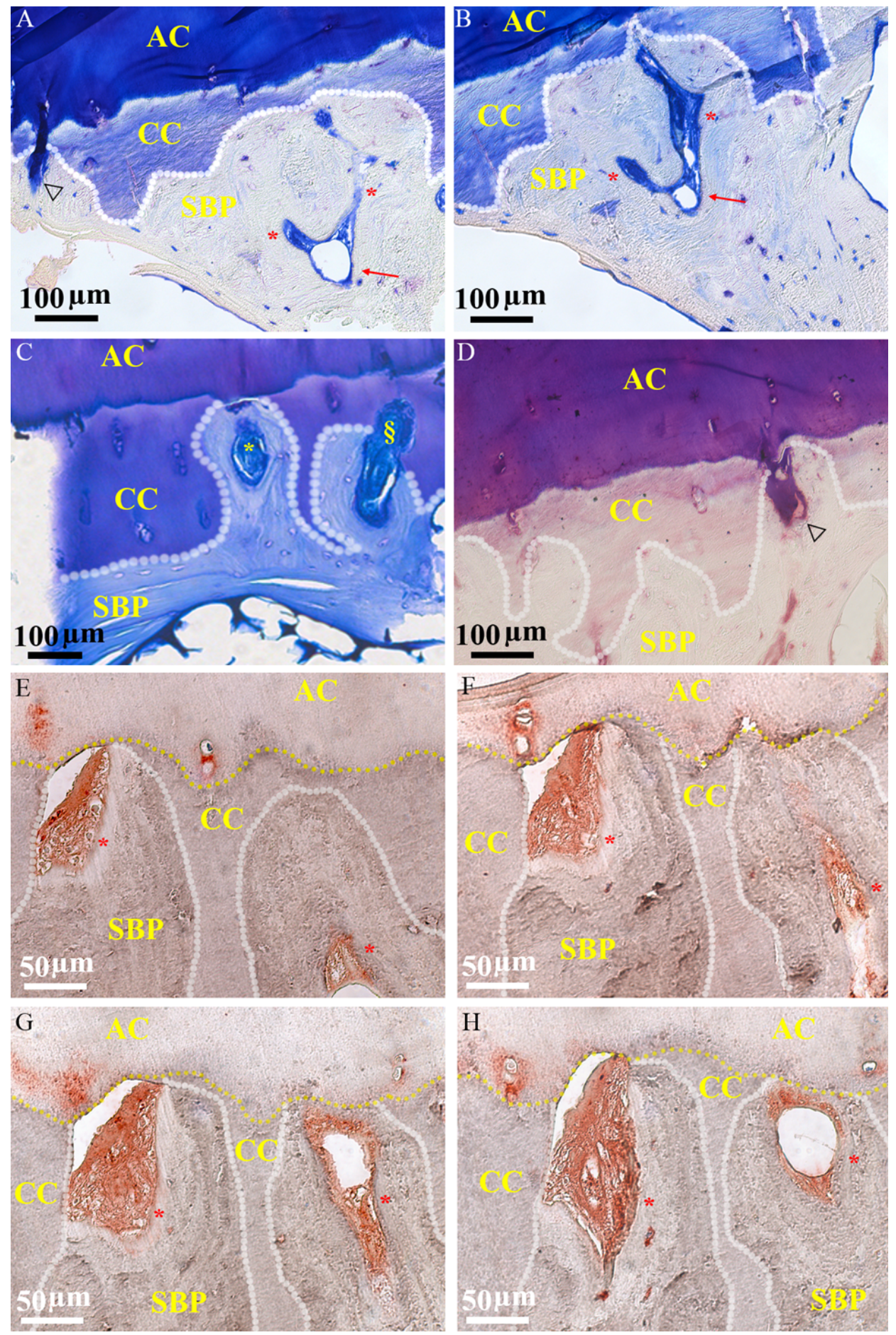

Figure 21. Depiction of the osteochondral junction by histology / immunohistochemistry. (A)

Toluidine blue staining of a sample from the LBR showed prolongation of the AC beyond the CC layer (marked by an arrow head $\Delta$ ), as well the integration point (red arrow) of a dendric channel with two branches (red asterisks). (B) the coronally-adjacent slide of the A revealed the progression of the aforementioned dendric channel. (C) microchannels either cut through the CC layer (marked by $\S$ ) or circumvent it (yellow asterisk). (D) the H\&E staining again showed an occasional prolongation of the AC $(\Delta)$ as well as the irregularly-shaped cement line (white dotted line). (E-F) The progression of two vesselcontaining microchannels (red asterisks) in four sequential slides are illustrated by vWF immunostaining. 
The von Willebrand Factor (vWF) immunostaining of four sequential slides showed the progression of two microchannels, both contained newly-formed microvessels, one was entirely in contact with the AC during the observed spatial range, while the other advanced from the medullary cavity and eventually abutted the basal AC with an approximate size of 50 (Figure 21E-H).

\subsubsection{The LBR is characterized by copious small microchannels, while large, infrequent, and elongated microchannels typify the SB microstructure in the peripheral rim of the femoral head}

Several structural parameters of the SB microchannel system were quantified in the healthy human femoral heads. Based on the loading areas on the joint, distinct diagrams for the local density of the microchannels (CMMC number) were observed from tidemark to deeper trabecular bone. Namely, the number of CMMC was consistently higher in the LBR compared to other areas on the joint (Figure 22A). The decreasing steep slope of the profile after $150 \mu \mathrm{m}$ from the tidemark corresponded to the integration of the CMMC in close proximity to the cortical end-plate. Beyond that and at deeper distances from the tidemark, the values represent the cavities between the trabeculae and did not correspond to the SB microchannels. Particularly interesting for us were the CMMC that crossed the calcified cartilage layer and were bound to the lower margin of the AC by physical contiguity. Therefore, I focused on the mean CMMC number in the uppermost $50 \mu \mathrm{m}$ of the SB of different loading areas (Figure 22B). A significant region-based effect was observed based on the repeated measures one-way ANOVA (Wilks' Lambda $=0.052, \mathrm{~F}$ $\left.(2,8)=27.12, p=0.012, \eta^{2}=0.948\right)$. Follow up pairwise comparisons revealed that the CMMC number in the LBR (mean $\pm \mathrm{SD}, 9.85 \pm 3.261 / \mathrm{mm}^{2}$ ) was higher compared to the $\operatorname{NLBR}\left(4.08 \pm 1.581 / \mathrm{mm}^{2} ; p=0.002\right)$, and the PR $\left(4.94 \pm 1.741 / \mathrm{mm}^{2} ; p=0.006\right)$. The local density of the microchannels at the top $50 \mu \mathrm{m}$ of the SB was not statistically different between the NLBR and the PR.

A similar region-dependency in the size distribution profiles of the SB microchannels was revealed. Pairwise comparisons showed in particular that the maximum caliper diameter (Feret) at the AC-SB interface (upper $50 \mu \mathrm{m}$ ) was increased from the LBR (mean $\pm \mathrm{SD}$, $55.04 \pm 4.2 \mu \mathrm{m})$ to the NLBR $(74.25 \pm 9.34 \mu \mathrm{m} ; p=0.02)$ and the PR $(91.30 \pm 12.47 \mu \mathrm{m} ; p$ $=0.0004$; Figure 22D). Analogous variations were found for the minimum microchannel caliper diameter, where LBR (mean $\pm \mathrm{SD}, 36.22 \pm 2.28 \mu \mathrm{m}$ ) exhibited the smallest MinFeret while the minimum microchannel diameter was greater at PR $(57.16 \pm 8.58 \mu \mathrm{m}$; 
$p=0.003$; Fig. Figure 22F). For the NLBR, an average MinFeret of $46.47 \pm 6.39 \mu \mathrm{m}$ was calculated in the uppermost $50 \mu \mathrm{m}$, which was bigger than the $\operatorname{LBR}(p=0.12)$ and smaller than the PR $(p=0.09)$, albeit insignificantly in both cases.
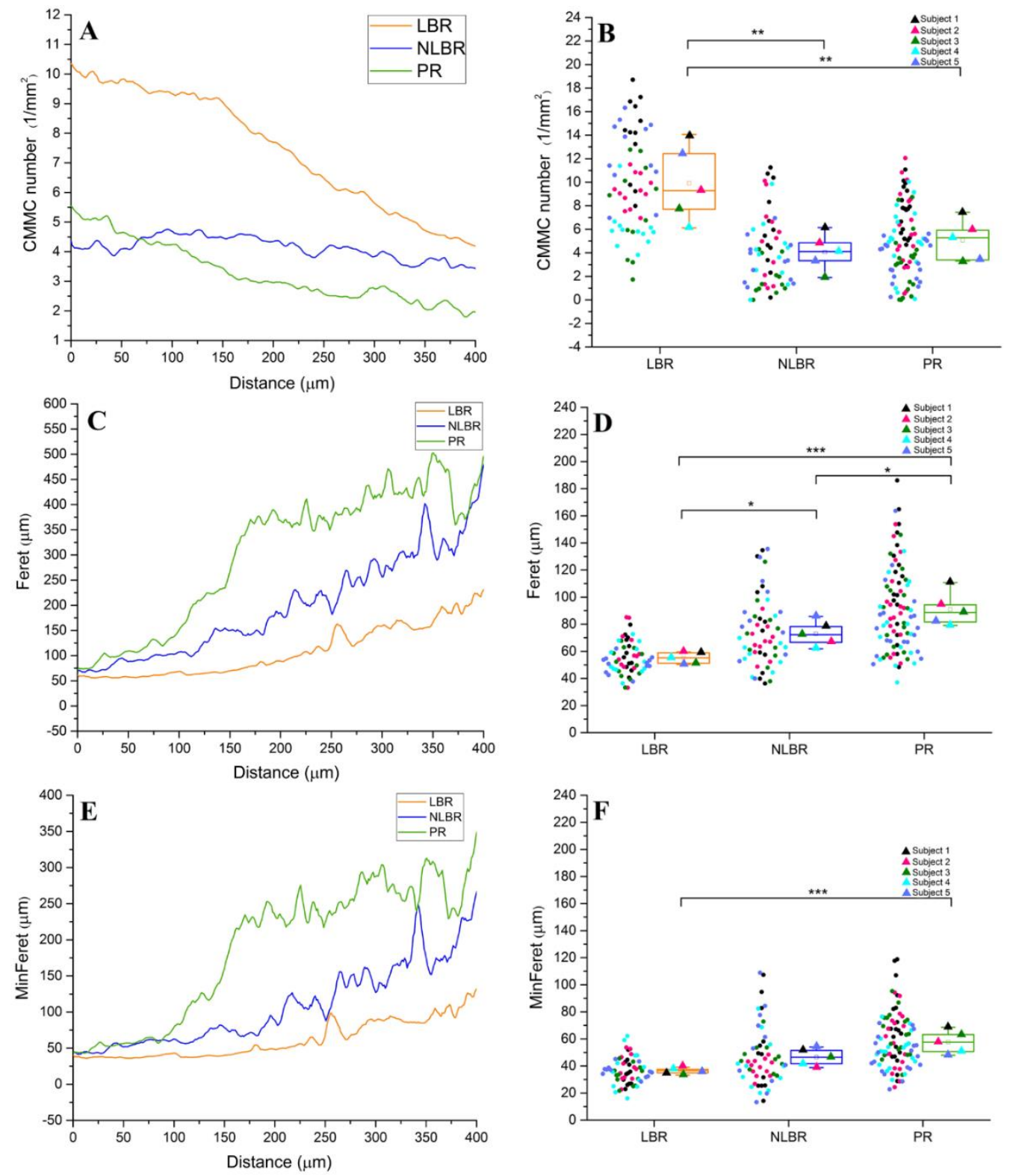

Figure 22. Quantitative analysis of the CMMC in the healthy human femoral heads. The changes of the number of microchannels per $\mathrm{mm}^{2}(\mathrm{~A})$, Feret $(\mathrm{C})$, and MinFeret (E) were illustrated versus distance from the tidemark in different loading areas of the joint. At the AC-SB junction (upper $50 \mu \mathrm{m}$ ), the alterations of the CMMC number (B) Feret (D) and MinFeret (F) are depicted as boxplots. Each circle in the immediate vicinity of the boxplots represented the mean value of a measuring point, color-coded to its corresponding subject. The total number of the measuring points for the LBR, NLBR, and the PR were 60, 60 , and 95, respectively. The triangles overlying the boxplots denote the mean values of each subject in different loading areas. Repeated measures one-way ANOVA followed by Bonferroni post-hoc test $* p<0.05 ; * * p<0.01 ; * * * p<0.00$ 
Region-dependent differences in the morphology of the SB microchannels were noted as well (Figure 23A). The porous structures were generally round and circular in the LBR, while getting increasingly elongated and irregular at the rim of the joint. At the AC-SB interface of the load-bearing region, in particular, we found a mean \pm SD Circ. value of $0.83 \pm 0.03$, while the values were significantly declined in the NLBR $(0.75 \pm 0.02 ; p=$ $0.002)$ and PR $(0.69 \pm 0.02 ; p<1 \mathrm{e}-4$; Figure 23B). Likewise, the difference between the NLBR and the PR was significant $(p=0.017)$.
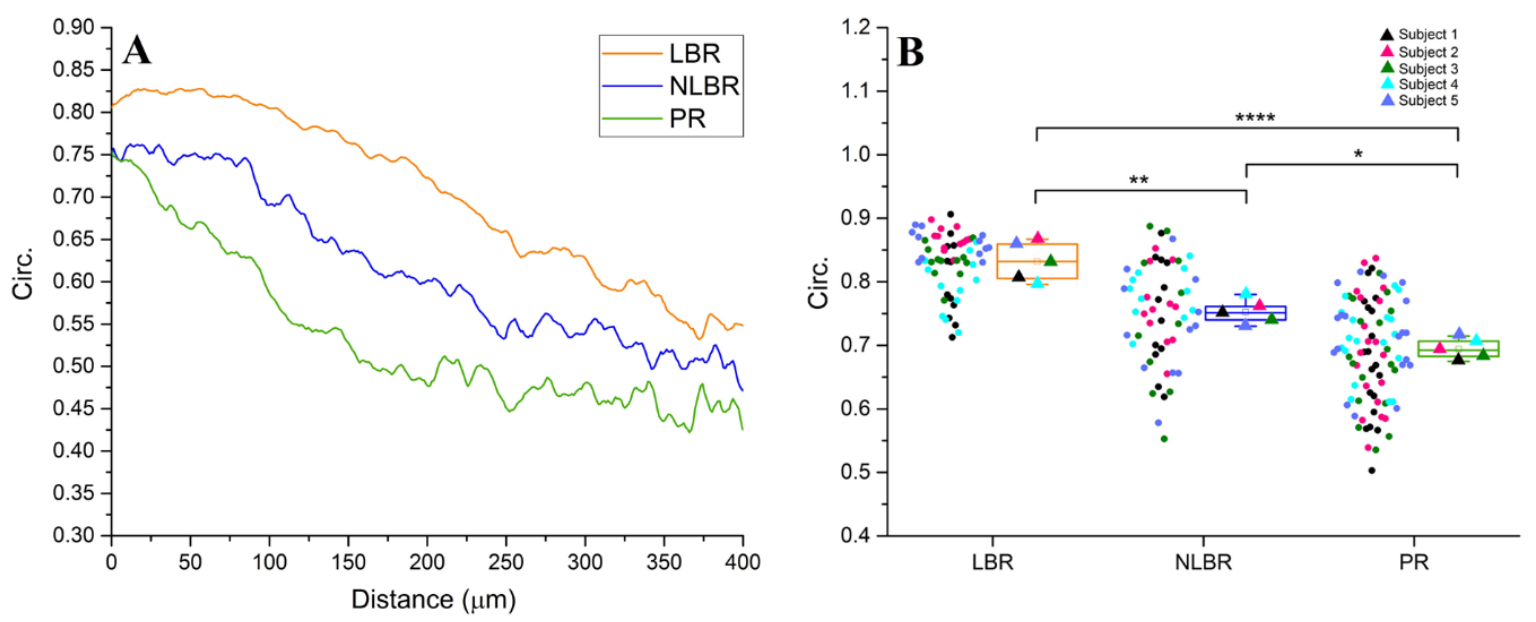

Figure 23. Region-based morphological changes of the SB microchannels in the femoral head. (A) Changes of the circularity index (Circ.) versus distance from the tidemark are profiled for each region. (B) Boxplots showed the mean Circ. at the AC-SB interface (uppermost $50 \mu \mathrm{m}$ ). Color-codes represented the measuring points extracted from each subject. Statistical significance was derived from repeated measures one-way ANOVA followed by Bonferroni post-hoc test. * $p<0.05$; ** $p<0.01 ; * * * * p<0.0001$

\subsubsection{Cartilage thickness is positively associated with the SB thickness, CMMC number, and circularity.}

Correlation studies at the AC-SB interface (upper $50 \mu \mathrm{m}$ ) showed moderate association of the AC thickness with several parameter of the SB microarchitecture. The results are presented in Table 7. SB thickness, CMMC number, and Circ. were positively correlated with the AC thickness, while the size of the microchannels demonstrated a negative correlation with the thickness of the overlying cartilage. 
Table 7. Correlation of the AC thickness with different variables of the SB microarchitecture

\begin{tabular}{cccc}
\hline AC thickness vs. & Pearson's r & R-Square (COD) & $p$ (ANOVA) \\
SB thickness & 0.48 & 0.24 & $<1 \mathrm{e}-13$ \\
CMMC number & 0.46 & 0.21 & $<1 \mathrm{e}-11$ \\
Feret & -0.43 & 0.19 & $<1 \mathrm{e}-10$ \\
MinFeret & -0.36 & 0.13 & $<1 \mathrm{e}-7$ \\
Circ. & 0.61 & 0.37 & $<1 \mathrm{e}-38$ \\
\hline
\end{tabular}




\subsection{SB microchannels in early osteoarthritis}

The results presented in this section are partially based on the following filed US patent with the University Medical Center Göttingen

Taheri S, Schilling AF: Microchannels in subchondral bone and membranes comprising same for the treatment of osteoarthritis. USPTO No.16/282,444 (filed)

\subsubsection{Partial thickness defect and early cartilage fibrillation occurs prevalently at the inferoposterior of the femoral head.}

The three-dimensional models of the femoral heads were generated by means of a photogrammetry technique, and the area of early cartilage degeneration and partial thickness defect were superimposed on a 3D representation of the femoral head's surface (Figure 24). In total, 116 cartilage-bone biopsies were extracted, where the majority of them were located at the NLBR of the joint (73 out of 116; $63 \%$ of the total measuring points). In particular, partial thickness defect was prevalent inferoposteriorly, in the vicinity of the fovea capitis. The second most recurrent area of early cartilage fibrillation was at the outermost peripheral rim of the joint (32 measuring points), while the LBR showed the least frequency of partial thickness defect (11 measuring points).

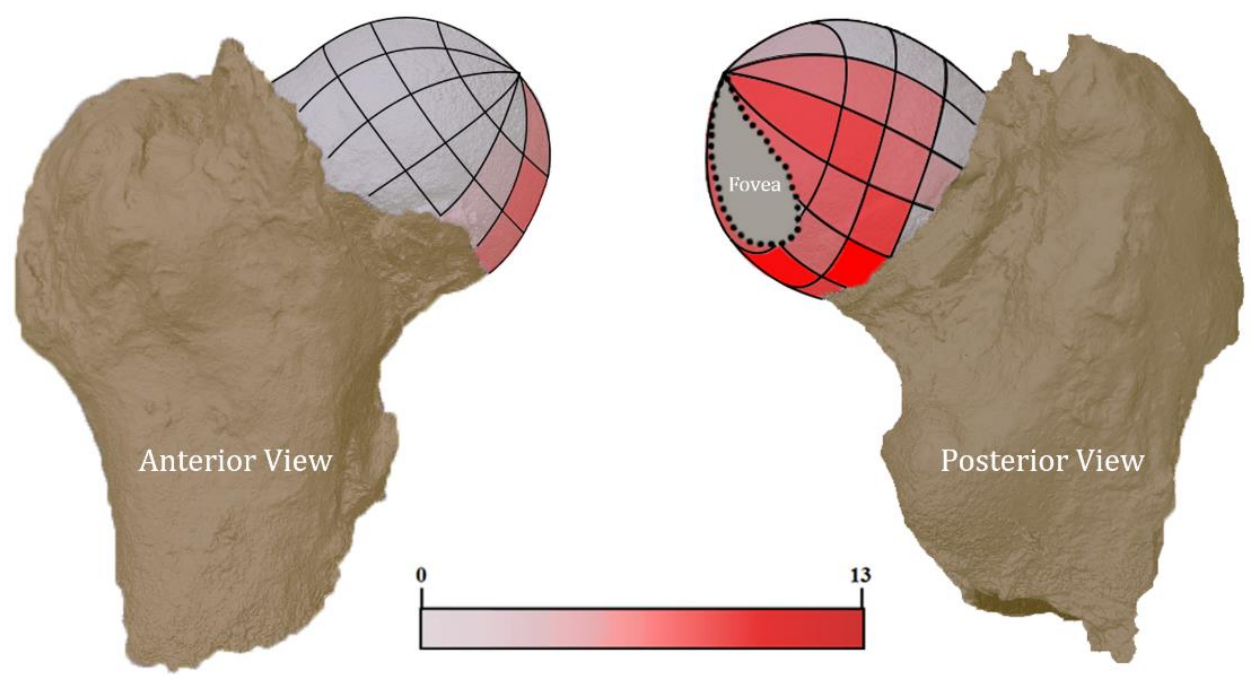

Figure 24. The distribution of early AC fibrillation and partial thickness defect on the femoral head. A maximum of four measuring points per sector was extracted from each femoral head in case of typical early OA cartilage defects. The color scale is based on the total number of measuring points per sector in five femoral heads. 73, 32, and 11 measuring points with early AC lesions were identified and extracted from the NLBR, PR, and LBR, respectively. 


\subsubsection{The thickness of SB is reduced in early $\mathrm{OA}$}

The 3D-recostructed models of exemplary osteochondral units are illustrated in Figure 25A, B. As expected, focal chondral defects and vertical fissures were observed in both samples that extended into the mid-zone of the AC (marked by asterisks). Due to the moderate erosion, the surface of the AC appeared as a discontinuous line, frequently showing hallmark signs of cartilage fibrillation and mild lesions (yellow arrowheads in Figure 25B).
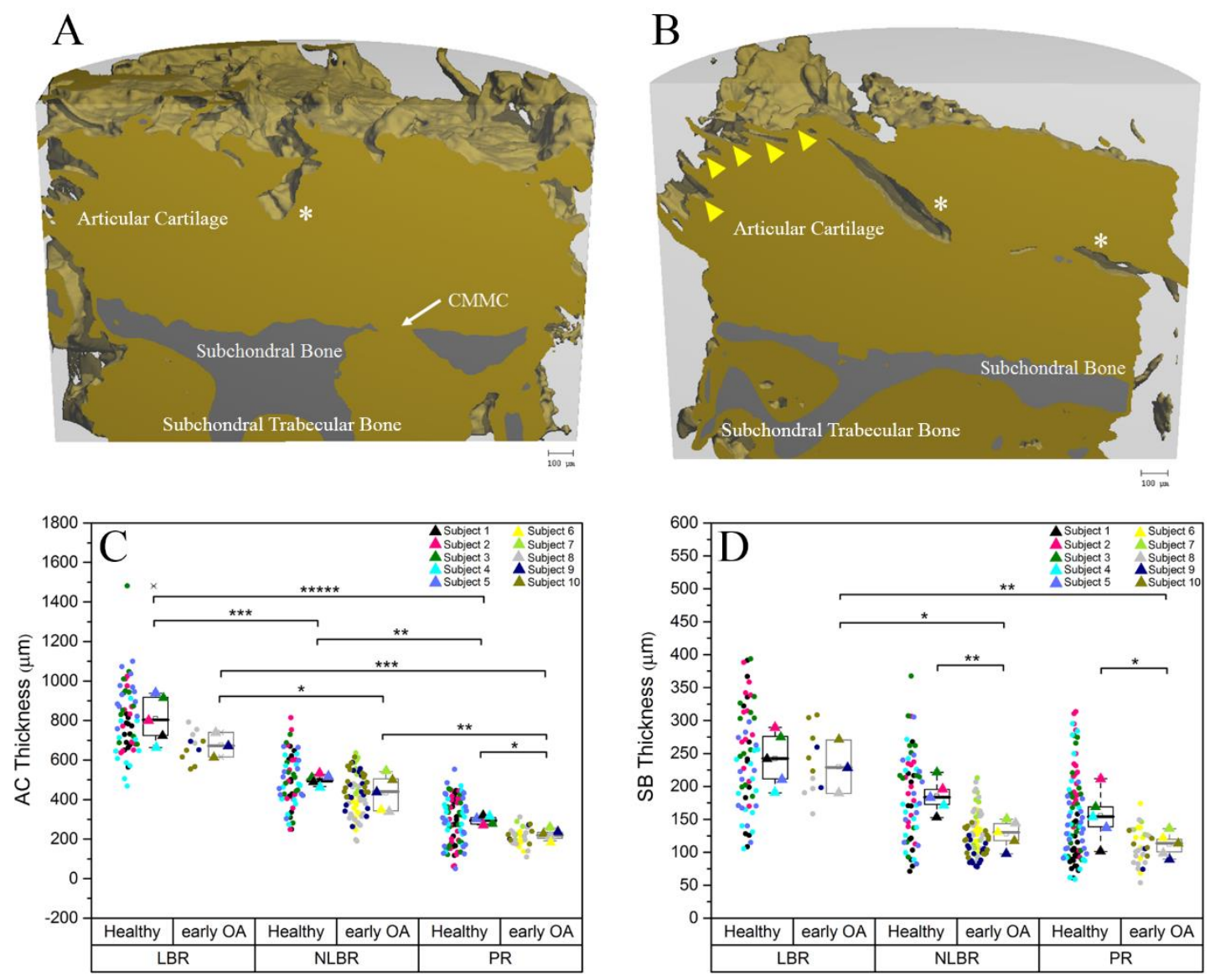

Figure 25. The early OA characteristics of the osteochondral unit in human femoral head. (A) The 3D-reconstructed model of a NLBR cartilage-bone biopsy showed uneven AC surface owing to the early pathological changes, while a partial chondral defect (marked by an asterisk) and a cartilage-bone marrow microchannel (white arrow) were highlighted. (B) Typical cartilage fibrillation was observed in the osteochondral unit of an exemplary LBR sample (yellow arrowheads) with vertical and horizontal fissures that advanced through the AC (white asterisks). (C) The early osteoarthritic changes of the AC thickness and (D) SB thickness were compared with healthy samples by Mixed-Design ANOVA followed by Bonferroni post-hoc test. Circles in close proximity to the boxplots represent the mean value of a measuring point, color-coded to their corresponding subjects. The triangles overlying the boxplots signify the mean values of corresponding subjects in each loading region. Subjects 1-5 were healthy while subjects 6-10 had early osteoarthritic chondral characteristics. 
The Mixed-Design ANOVA analysis revealed a significant effect of the loading areas on AC thickness $\left(p=0.00012, \eta^{2}=0.861\right)$. Follow-up pairwise assessments showed a similar region-based AC distribution of the early OA compared to the healthy femoral heads, as LBR had the highest AC thickness (mean \pm SD, 671 $\pm 62 \mu \mathrm{m}$ ), while the value decreased significantly in the NLBR $(425 \pm 91 \mu \mathrm{m} ; p=0.0274)$ and the PR of the joint $(213 \pm 25 \mu \mathrm{m}$; $p=0.00048$; Figure 25C). As for the effect of the health-state, we observed obvious trends of AC thickness reduction for each region compared to their corresponding healthy groups, which was statistically significant only at the peripheral rim of the joint $(p=$ 0.047). No interaction effect between loading-areas and the health-state was detected for the AC thickness. Likewise, it was found in the early OA samples that SB thickness was significantly thicker in the LBR $(233 \pm 40 \mu \mathrm{m})$ compared to the NLBR $(126 \pm 20 \mu \mathrm{m} ; p=$ $0.032)$ and the PR $(110 \pm 17 \mu \mathrm{m} ; p=0.0026$; Figure 25D). Pairwise comparisons showed significant effect of the health-state on the subchondral bone. In particular, thinning of the SB was observed in the NLBR (32\% reduction; $p=0.0081)$ and the PR $(28 \%$ reduction; $p=0.0445$ ) of pathological samples compared to their healthy counterparts. Again, no interaction effect between the region and the health-state was observed for the SB thickness (region*health-state; $p=0.066$ ).

\subsubsection{The CMMC maintain their location-dependent characteristic in early OA}

The cartilage-bone marrow microchannel connectors were frequently observed in the early osteoarthritic regions as well. The representative 3D-reconstructed views (Figure 26) showed a noticeable location-specific distribution pattern of the microchannels, which was in essence comparable to that of healthy femoral heads (see Figure 19); albeit with a much higher CMMC size in all regions. In general, several small-sized microchannels advanced to the uppermost SB surface in the load-bearing region (Figure 26A, B). The negative 3D-model of the osteochondral junction showed that the channels were continuous, providing a direct connection between the deeper subarticular area and the cartilage (Figure 26C). The local density of the CMMC appeared to be reduced in the NLBR compared to the LBR, with a varying combination of small, medium, and large channel sizes (Figure 26D-F). At the peripheral rim of the joint, very large and irregularlyshaped gap formations in the SB were observed (Figure 26G-H). These gaps had large contiguity areas with both the overlying cartilage and the underlying subarticular region (marked by red surfaces in Figure 26I). 

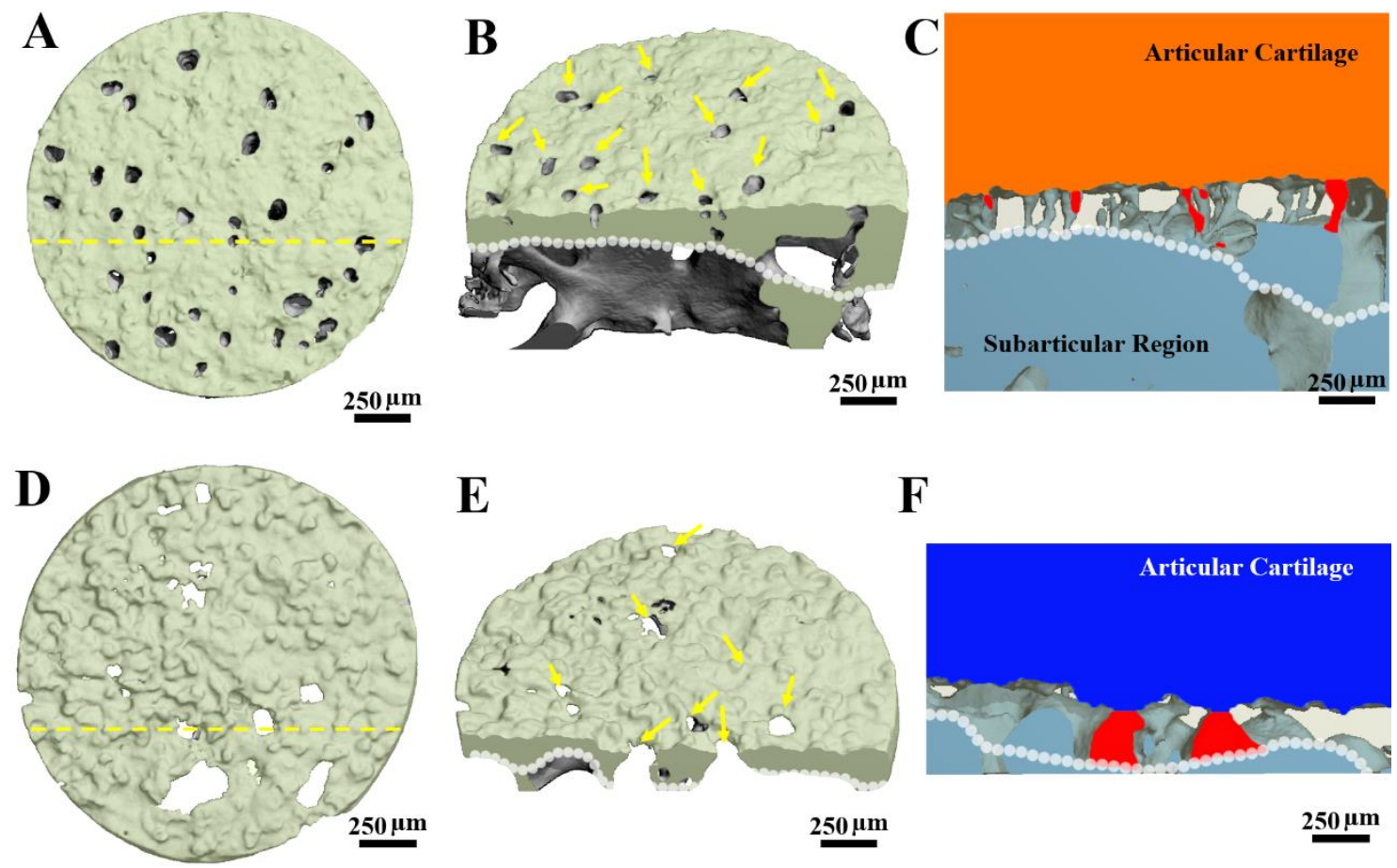

$\mathbf{F}$
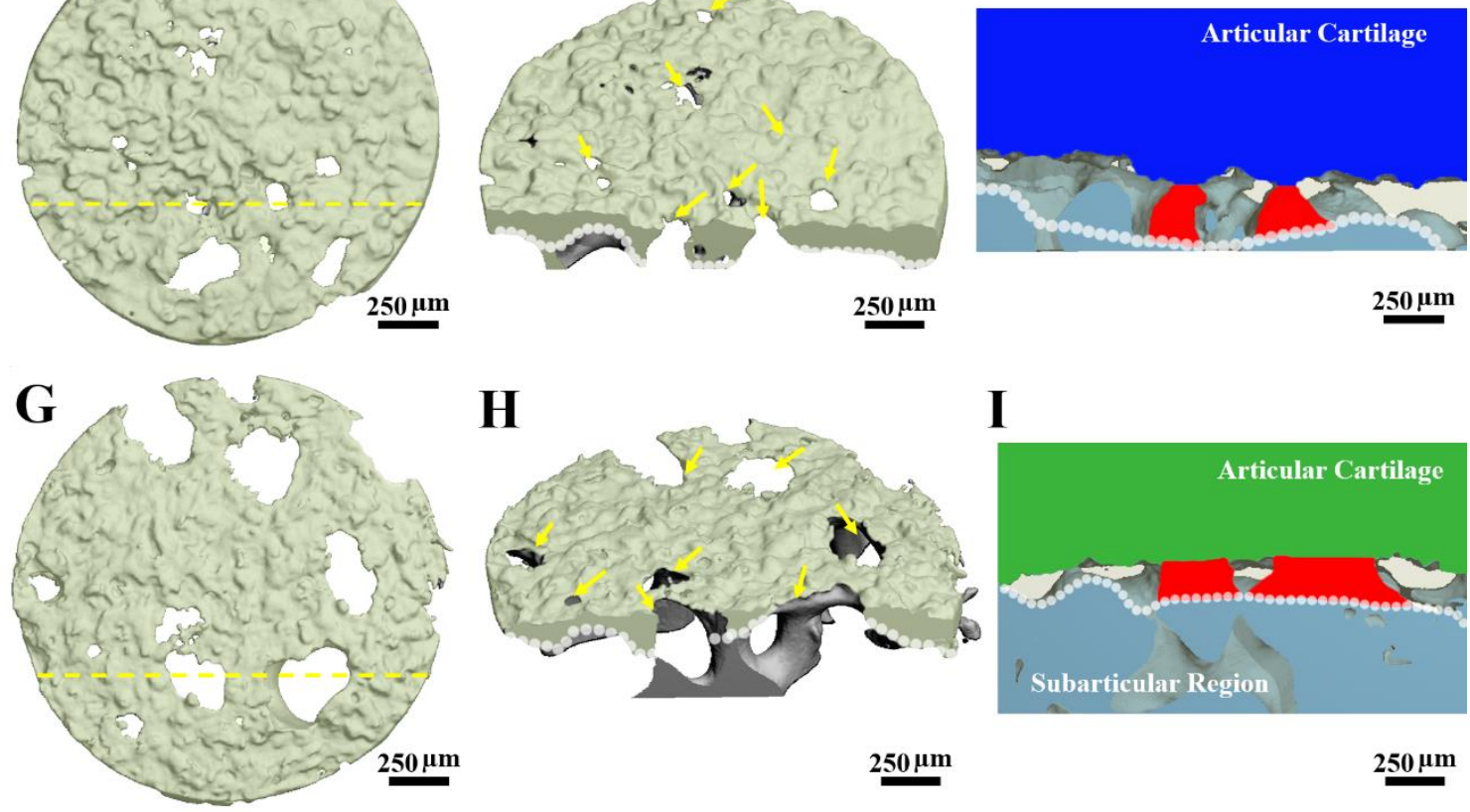

I

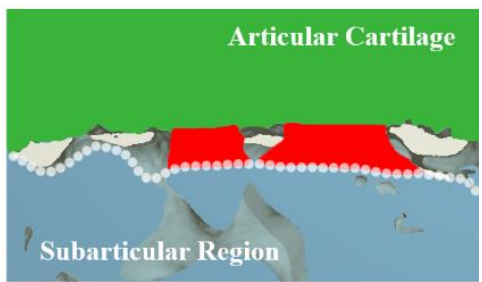

$250 \mu \mathrm{m}$

Figure 26. 3D representation of the SB microchannel network in early OA femoral heads. (A) The superior view of an exemplary sample from the load-bearing region revealed abundant CMMC on the uppermost SB surface. Yellow dash line represents an arbitrary coronal plane of sectioning that demonstrates (B) the cross-section of the SB, and virtually cuts some of the microchannels (marked by yellow arrows). (C) The negative 3D model of the same LBR cross-section shows the AC in orange, osseous structures in transparent, and the CMMC in blue-grey. The microchannels were visible throughout the entire thickness of the SB, while discrete microchannels cut by the plane of sectioning were marked in red. For the non-load-bearing region and the peripheral rim, representative views of the superior, coronal, and the inverted osteochondral unit are presented in D-F and G-I, respectively.

\subsubsection{The local density of CMMC does not change in early OA but their size is increased}

The structural parameters that were introduced to assess the SB microarchitecture (see 3.2.5) were quantified for the early OA samples, and the results were compared with the healthy human femoral heads. As before, I concentrated on the AC-SB interface (uppermost $50 \mu \mathrm{m}$ of the SB) to assess the microchannels that advanced through the CC 
layer and were bound to the basal cartilage by physical contiguity. The Mixed-Design ANOVA revealed a significant region-dependent effect of the mean CMMC number in early OA $\left(p=0.0078, \eta^{2}=0.857\right)$. Pairwise comparisons showed the region-dependency of the CMMC number in early OA samples, where LBR had consistently a higher local CMMC density (mean $\pm \mathrm{SD}, 10.5 \pm 1.71 / \mathrm{mm}^{2}$ ) than the NLBR $\left(5.4 \pm 2.71 / \mathrm{mm}^{2} ; p=0.03\right)$ and the PR $\left(4.5 \pm 2.31 / \mathrm{mm}^{2} ; p=0.0017\right.$; Figure 27A). Similar to the results of the healthy femoral heads, no statistical difference between the CMMC number of the early OA samples in the NLBR and the PR was observed. Likewise, health-state (healthy vs. early $\mathrm{OA})$ did not influence the CMMC number in any region, hence, no interaction effect for the CMMC number was detected.

Similar to the healthy SB microchannels, it was observed that the size of the CMMC in early OA is highly region-dependent ( $p=6 \mathrm{e}-5, \eta^{2}=0.933$; Figure 27B). In particular, the caliper Feret diameter at the AC-SB interface was significantly increased from the LBR (mean $\pm \mathrm{SD}, 83.4 \pm 9.1 \mu \mathrm{m})$ to the NLBR $(134.2 \pm 19.2 \mu \mathrm{m} ; p=0.0025)$ and the PR $(152.9 \pm 38.4 \mu \mathrm{m} ; p=0.007)$. More interestingly, pairwise comparisons of the healthy and early OA samples showed significant effect of the Feret size in all regions; Namely, 51\% $(p=0.016), 81 \%,(p=0.00064)$ and $89 \%(p=0.005)$ increase were observed in the LBR, NLBR, and the PR, respectively. Consequently, a significant interaction was obtained between the region and health-state for the CMMC size (region*health-state; $p=0.02$ ). For the minimum CMMC caliper diameter in early OA samples, similar region-dependent differences were observed where the smallest MinFeret was at the LBR (mean $\pm S D$, $53.6 \pm 10.0 \mu \mathrm{m})$, and significantly increased at the NLBR $(85.9 \pm 17.3 \mu \mathrm{m} ; p=0.024)$ and the PR $(96.8 \pm 16.4 \mu \mathrm{m} ; p=0.0033$; Figure 27C). Additionally, the MinFeret showed a clear increasing trend in early OA compared to the corresponding healthy groups, which was significant in the NLBR $(85 \%$ increase; $p=0.00286)$ and the PR $(68 \%$ increase; $p=$ 0.019 ). The interaction of the region and health-state was not statistically significant ( $p=$ $0.082)$.

The morphology of the SB microchannels showed higher variance compared to the healthy groups, and as a result, the measuring points were more scattered. The MixedDesign ANOVA showed a significant region-dependent effect of the mean Circ. in early OA $\left(p=0.0019, \eta^{2}=0.515\right)$. However, pairwise comparisons showed a significant difference only between the LBR and PR $(0.79 \pm 0.07$ vs. $0.66 \pm 0.04 ; p=0.026)$. When early OA groups were compared to their healthy counterparts, decreasing trends were observed for all groups, which were, however, not significant due to the aforementioned 
variance. In general, the CMMC had relatively a round and circular shape in the LBR, while getting increasingly elongated and irregular at the rim of the joint (Figure 27D).
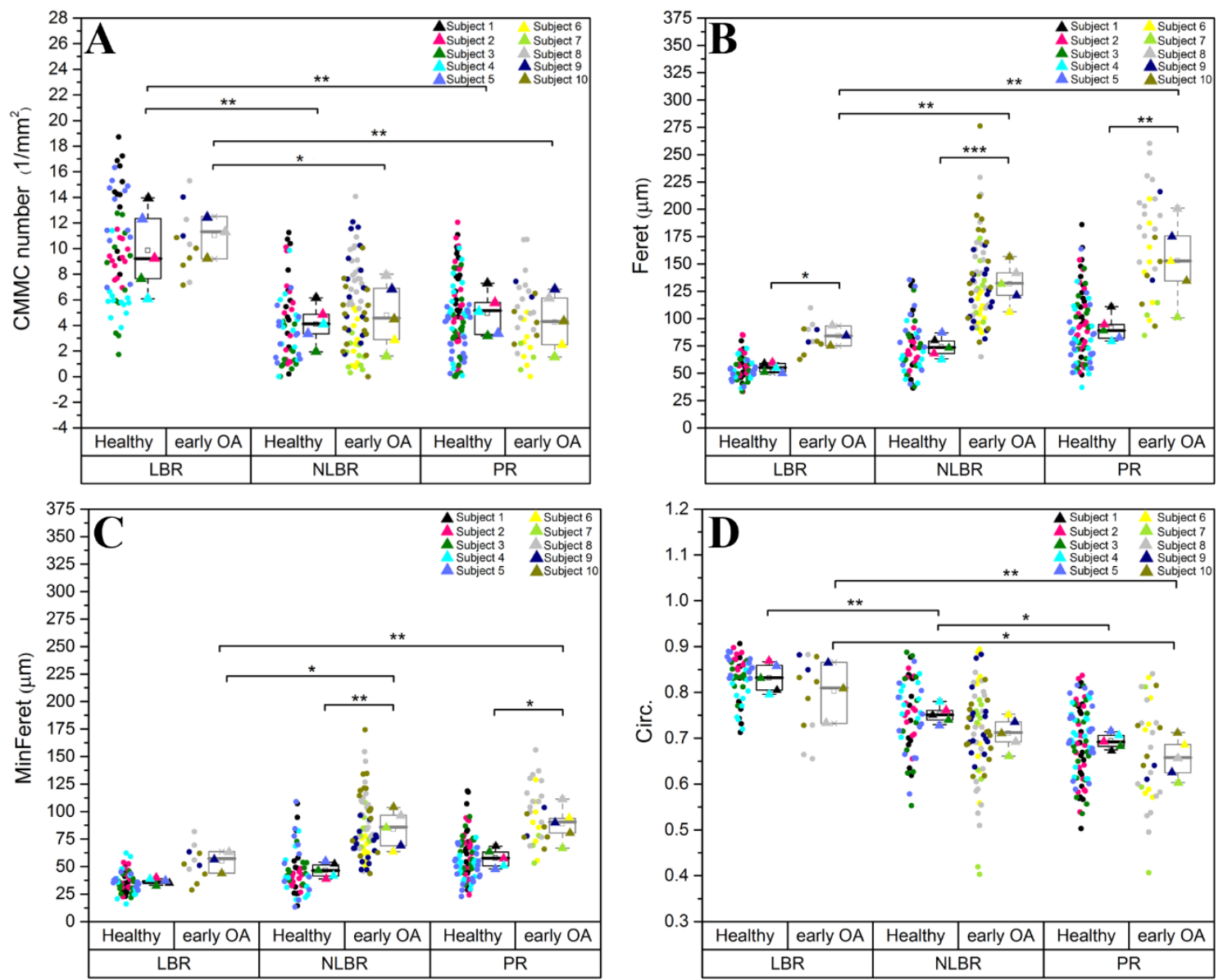

Figure 27. Quantitative analysis of the CMMC in the early OA human femoral heads. The changes of the (A) CMMC number, (B) maximum caliper diameter (Feret), (C) minimum caliper diameter (MinFeret),

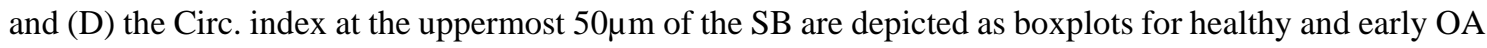
human femoral heads in different loading areas. Each circle in the immediate proximity to the boxplots represents the mean value of a measuring point, color-coded to its corresponding subject. The total number of the early OA measuring points for the LBR, NLBR, and the PR were 11, 73, and 32, respectively. The triangles overlying the boxplots denote the mean values of each subject in different loading areas. MixedDesign ANOVA followed by Bonferroni post-hoc test was conducted to compare healthy and early OA samples in their corresponding groups $* p<0.05 ; * * p<0.01 ; * * * p<0.001$. 


\subsection{Early and late subchondral bone changes in a traumatic OA model}

The results presented in this section are based on the following published paper:

Muschter D*, Fleischhauer L*, Taheri S*, Schilling AF, Clausen-Schaumann H, Grässel S (2020): Sensory neuropeptides are required for bone and cartilage homeostasis in a murine destabilization-induced osteoarthritis model. Bone 133, 115181

(* These authors contributed equally)

\subsubsection{DMM surgery induces progressive cartilage degradation}

The progressive degradation of the articular cartilage was studied in the coronal slices of Safranin-O stained murine knee joints in cooperation with the University of Regensburg. Corresponding histological sections of the WT, Tac1-/- and $\alpha \mathrm{CGRP}-/-$ mice are presented at early (two weeks), and late (8 weeks) stages after the DMM surgery, and at 2 weeks post-operative after the Sham surgery (Figure 28). As early as two weeks after the induction of osteoarthritis, proteoglycan loss of the AC was observed in the medial tibial plateau. By 8 weeks, cartilage degradation manifested late-stage OA characteristics and local SB exposure. Full OARSI scores for different timepoints post-operative can be seen in (Muschter et al. 2020).

\subsubsection{Traumatic $\mathrm{OA}$ induction leads to thickening of the calcified cartilage}

By combining a method based on different greyscale values and modified Seeded Region Growing, the calcified cartilage was segmented and distinguished from its underlying subchondral bone plate. The corresponding color maps of the medial and lateral plateaus for the early ( 2 weeks post-operative) Sham and DMM mice from all genotypes are illustrated in Figure 29. For both condyles, the thickest CC was generally observed at the intercondylar tubercles. In the medial condyle of the DMM mice, a calcified layer of cartilage was already present on the new bone formations at the outermost rim of the knee joint, as indicated by the white arrows in Figure 29.8 weeks after the surgery, the regions of the intensified $\mathrm{CC}$ were extended to the contact areas on both condyles (Figure 30). In particular, the calcification on the newly-formed osteophytes at the medial plateau of the DMM mice were much more prominent compared to 2 weeks after surgery. 


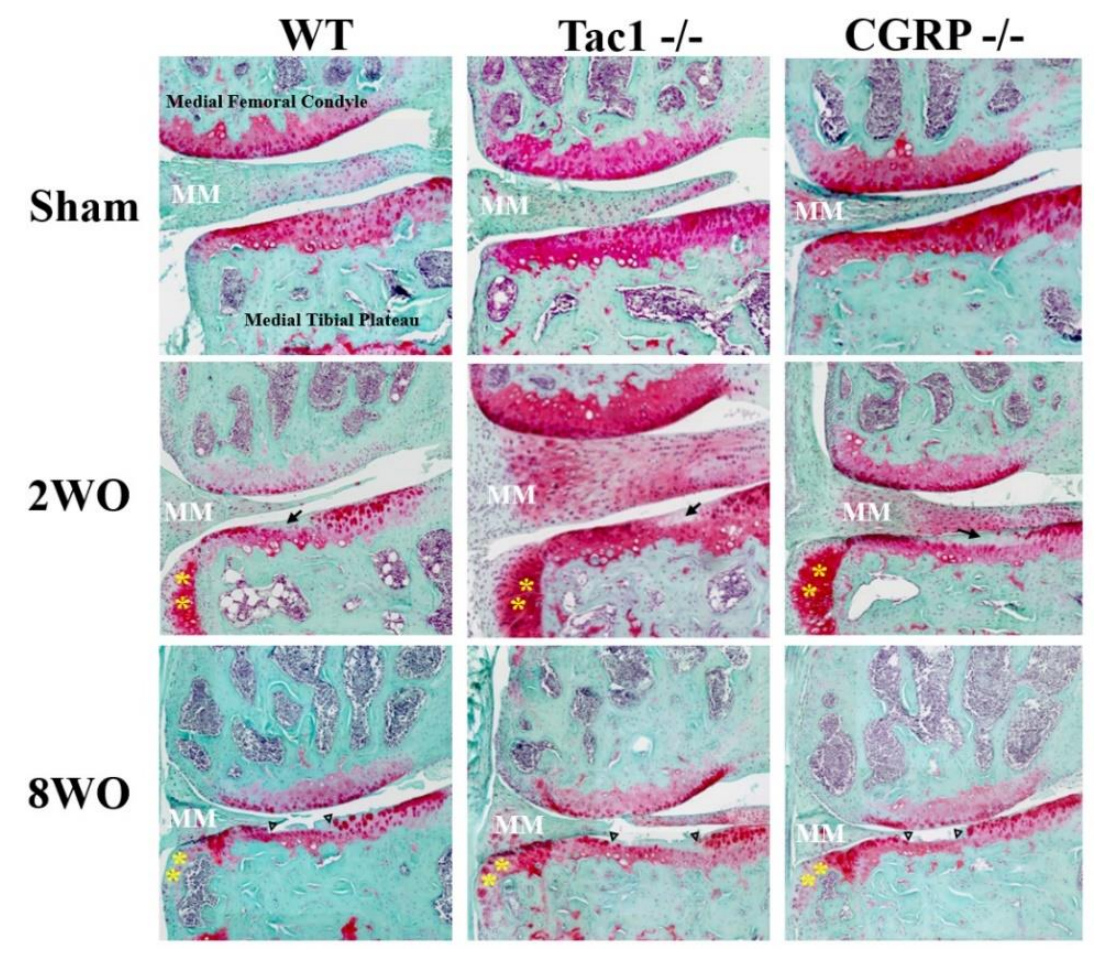

Figure 28. Safranin-O staining of the WT, Tac1-/-, and $\alpha$ CGRP-/- knee joints. The histological images are provided for the Sham mice after 2 weeks, and DMM mice at 2, and 8 weeks after the DMM operation. The integrity of the cartilage was assessed at the medial tibial plateau. Yellow Asterix (*) indicates osteophyte formation at the outermost edge of the joint, MM stands for medial meniscus, black arrows denote discoloration of the AC owing to the proteoglycan loss (2WO) and arrow heads represent late-stage cartilage degradation \{SOURCE: kindly provided by Dominique Muschter; unpublished $\}$

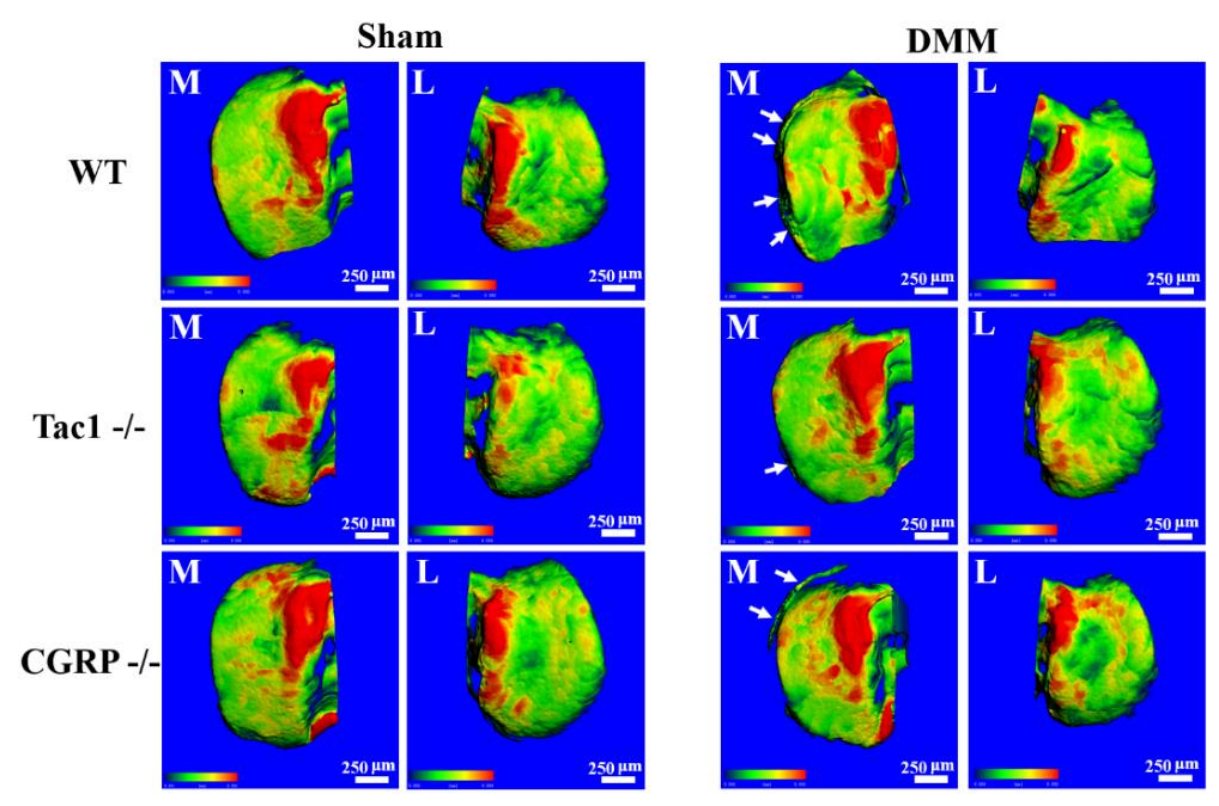

Figure 29. Calcified cartilage heat map of the mice tibial plateau 2 weeks after OA induction. Images were derived from a computational analysis based on grey-scale segmentation, and were normalized to a maximum color bar of 80 $\mu \mathrm{m}$ (Red $\gg$ green). M and L signify the medial and lateral condyles, respectively. The white arrows at the outermost edge of the DMM medial plateau show the CC layer on newly-formed osteophytes. 
The mean CC thickness of all genotypes was calculated in both condyles. In the early post-traumatic phase, the mean CC thickness of the DMM groups was unchanged compared to their Sham counterpart (Figure 31A). Within the DMM group, however, an increase was observed in the medial tibia calcified cartilage thickness in the aCGRP-/DMM mice compared to the WT DMM mice. The late post-traumatic phase was characterized by a significantly higher $\mathrm{CC}$ thickness in the medial condyle of all the DMM-operated mice compared to their respective Shams (Figure 31B). Likewise, the difference between the $\mathrm{CC}$ layer of the medial and lateral condyles became significant after the OA induction. In the lateral condyle of the DMM groups, the $\alpha \mathrm{CGRP}-/-$ mice showed the highest CC thickness, which might be due to an outlier within the group.
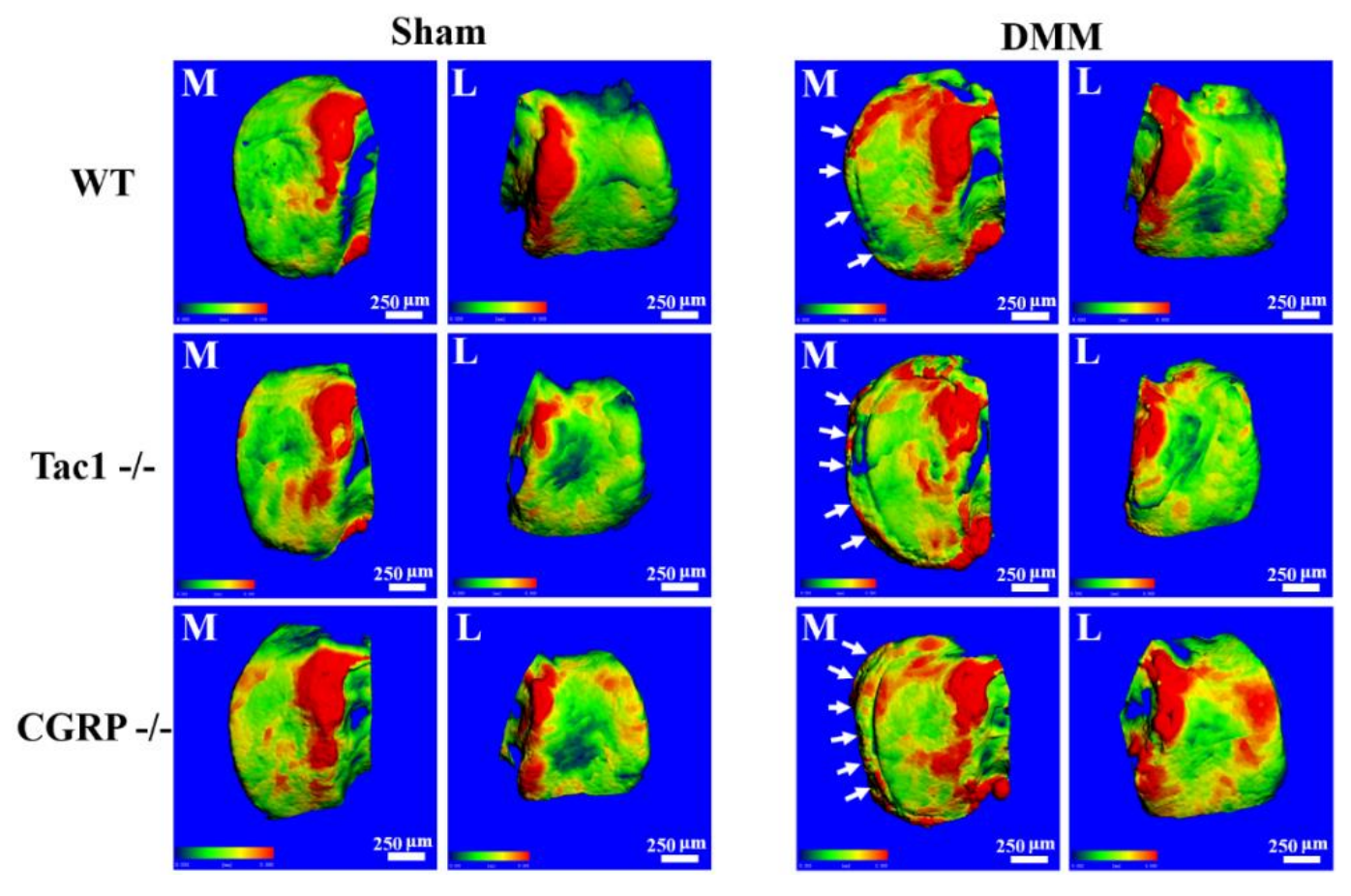

Figure 30. Calcified cartilage heat map of the mice tibial plateau 8 weeks after DMM surgery. Heat map representation of the $\mathrm{CC}$ layer was obtained from a gray scale-segmentation process. The color bar was normalized to a maximum valued of $80 \mu \mathrm{m}$ (Red $\gg$ green). M and L denote the medial and lateral condyles, respectively. The white arrows in the DMM model show the CC layer on newly-formed osteophytes at the outermost edge of the medial plateau. 

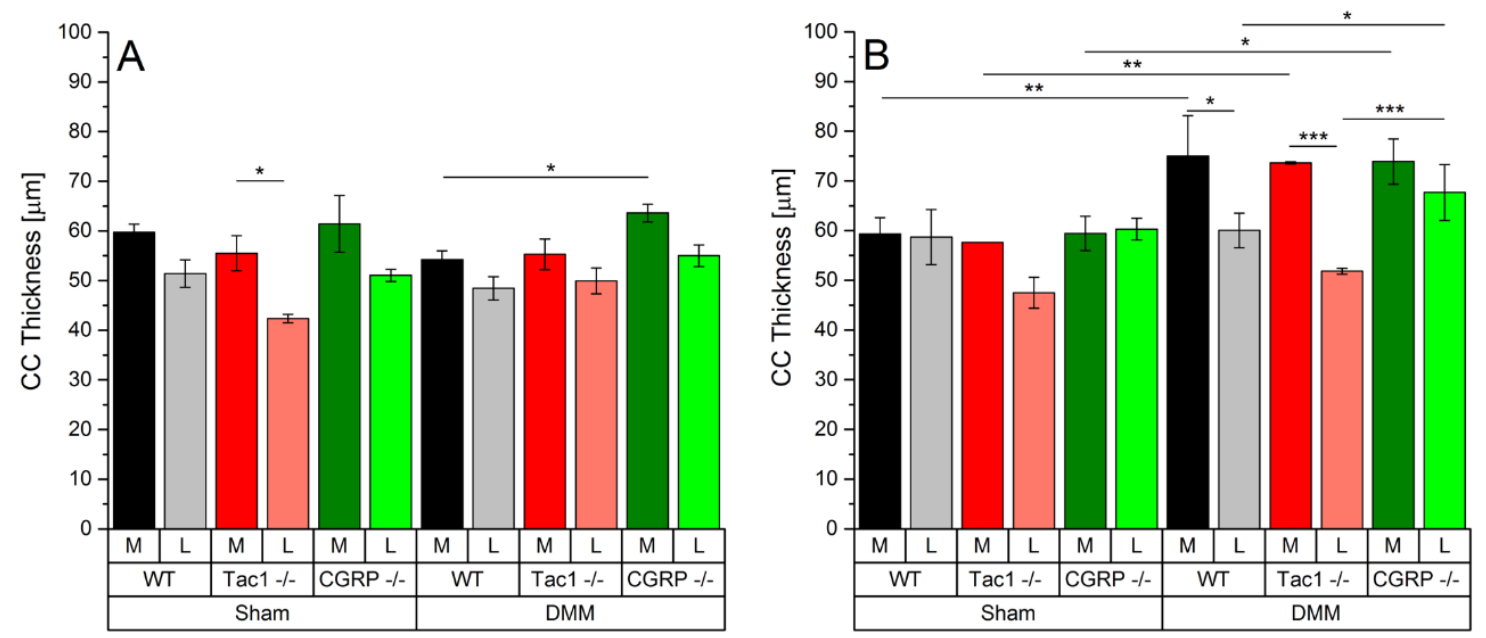

Figure 31. Diagrams of the mean CC thickness in the medial and lateral tibia plateaus. (A) at two weeks and (B) eight weeks after the DMM or Sham surgery. Statistical analysis for the WT, $\alpha \mathrm{CGRP}-/-$ and Tac1-/- mice were performed by two-way ANOVA followed by Tukey post-hoc test. * $p<0.05, * * p$ $<0.01, * * * p<0.001 . \mathrm{n}=3$.

\subsubsection{OA induction leads to irregular formation of osteophytes at the outermost rim of the medial tibial plateau}

The destabilization of the medial meniscus led to osteophyte formation at the outermost rim of the medial condyle. 2 weeks post-operative, the osteophytes were observed as lowmineralized new bone formations that covered the entire medial periphery of the joint (Figure 32A). With progression of the OA to eight weeks, the osteophytes were enlarged and well-integrated with the adjacent condyle. Hence, the length of the tibial plateau was measured as an indicator of osteophyte development. In the late post-traumatic phase, a significant increase of the length of the medial plateau was observed in all genotypes in comparison with their corresponding Shams. Nonetheless, no genotype-dependent difference was observed (Figure 32B). Generally, the lateral tibia was consistently wider in all groups in comparison with the respective medial tibia. In the Sham mice, the length of the medial condyle was in the same vicinity for all the three groups, while the lateral condyle of the $\alpha \mathrm{CGRP}-/-$ Sham was markedly reduced compared to the wildtype Sham.

The length of the lateral condyle in WT DMM group was unaltered in comparison with the WT Sham mice. On the other hand, a significant enlargement of the lateral condyle in the Tac1-/- DMM mice was observed compared to its Sham counterpart. A similar but smaller effect was observed for the $\alpha \mathrm{CGRP}-/-$ mice after DMM surgery, suggesting that the changes in the length of the lateral condyles were trauma-induced adaptations and were not influenced by the lack of sensory neuropeptides. 

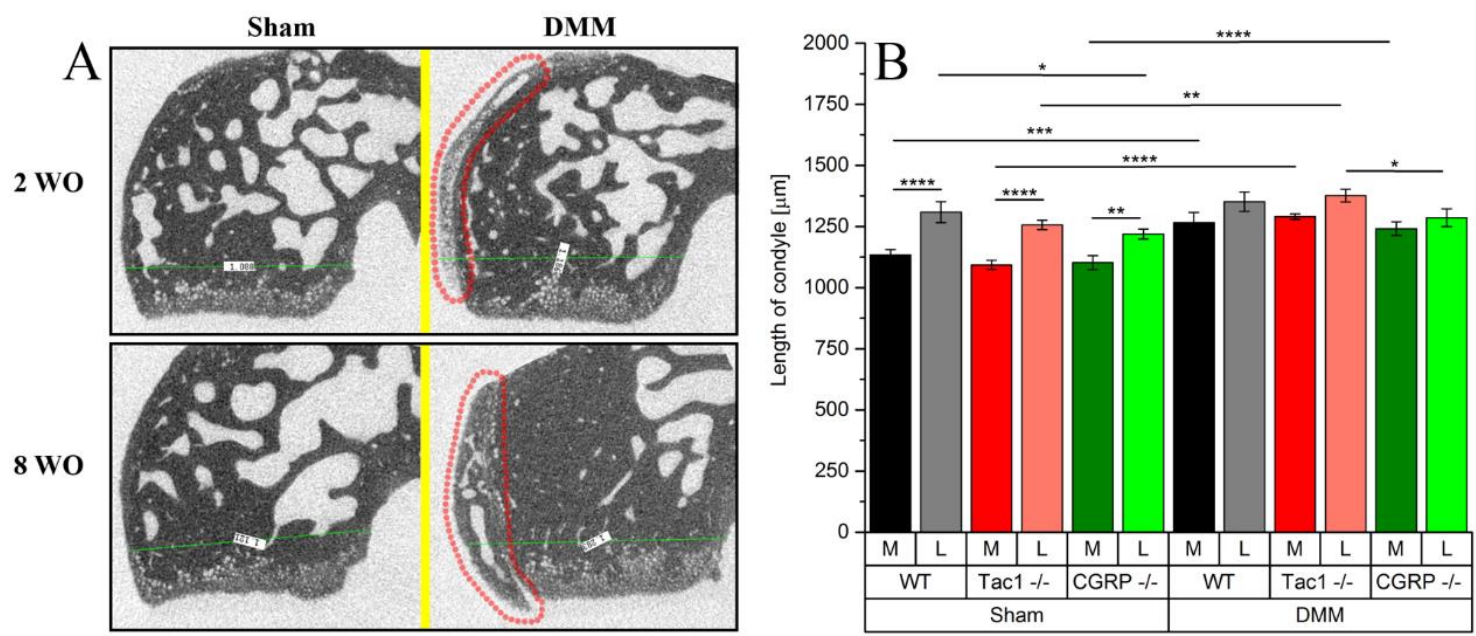

Figure 32. Osteophyte formation after DMM surgery in wildtype and neuropeptide-knockout mice.

(A) exemplary medial condyles of the Sham and DMM groups, two, and 8 weeks post-operative. The osteophytes are encircled by the red dotted shapes. The subchondral bone sclerosis after the DMM surgery could also be detected as indicated by the fusion of the trabecular structure within the SB. (B) 8 weeks postoperative, the lengthening of the medial and lateral plateaus was calculated as markers for the osteophyte formation as well as the adaptive mechanism within the knee joint. Two-way ANOVA followed by Tukey post-hoc test. $* p<0.05, * * p<0.01, * * * p<0.001, * * * * p<0.0001 . \mathrm{n}=3$.

\subsubsection{Medial subchondral bone sclerosis progresses during the course of traumatic OA}

The medial epiphysis of the knee joint was carefully examined using micro-CT imaging. The 3D-reconstructed models of the knee joints in the late post-traumatic phase, showing the femoral condyles, tibial plateaus, the growth plate, and part of the subchondral trabecular bone are illustrated in Figure 33A. Quantification of the bone volume fraction (BV/TV) in the epiphysis showed a trend of early sclerotic changes, as adjacent trabeculae became confluent in the medial subchondral bone that was, however, not statistically significant two weeks post-operative (Figure 33B). Eight weeks after the DMM surgery, evident fusion of the trabeculae and the cortical end-plate was observed. In particular, the SB sclerosis was more pronounced in neuropeptide-deficient groups as indicated by ca. $55 \%$ increase in the Tac1-/- and $\alpha$ CGRP-/- DMM mice in comparison with the wildtype DMM mice (Figure 33C). In the Sham mice, the BV/TV of the $\alpha \mathrm{CGRP}-/-$ mice was significantly higher in comparison with the WT Sham 8 weeks post-operative, indicating a clear bone phenotype even without the DMM surgery. Moreover, it was observed that in substance P-deficient mice, the medial epiphysis BV/TV was elevated after the DMM operation in comparison with the Sham mice. 


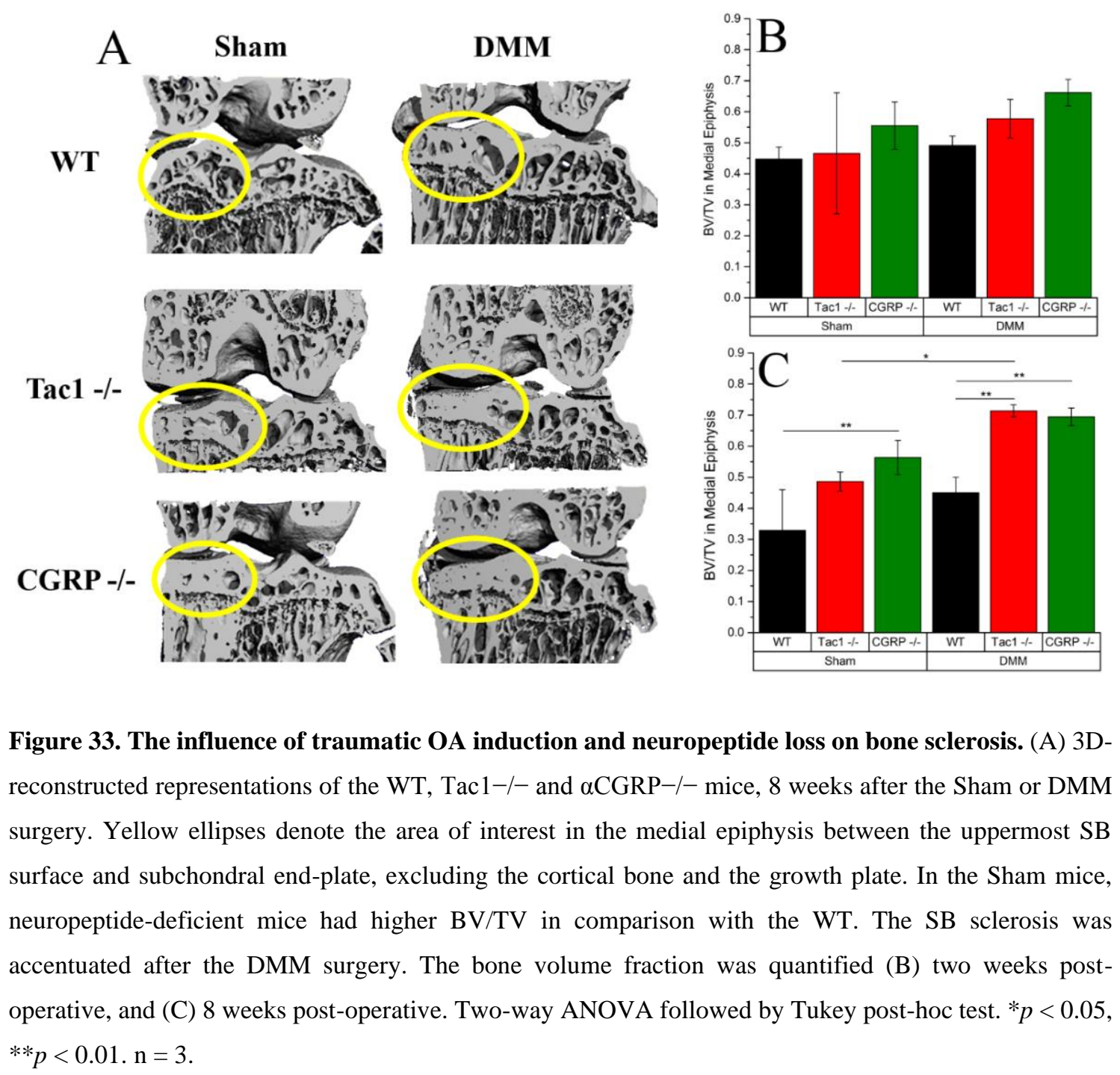

\subsubsection{Traumatic OA induces meniscal ectopic bone formation}

Increased meniscus ossification was observed as a strong phenomenon in the impact side of the DMM mice. Eight weeks after the OA induction, DMM mice exhibited ectopic spatial bone formation, which was more prominent in the neuropeptide-deficient mice compared to the WT mice (Figure 34A), the bone volume (BV) of the anterior meniscal ossicles was quantified in the early and late post-traumatic phases. Even though the interaction effect of the genotype (WT vs. Tac1-/- vs. $\alpha$ CGRP-/-) and the operation type (Sham vs. DMM) was not significant after 2 weeks, the mean BV of the pooled Sham groups were higher compared to the DMM (Figure 34B). With progression to 8 weeks, the bone volume of the meniscal ossicles was significantly increased in all DMM mice in comparison with their corresponding Shams (Figure 34C). Quantification of the BV showed that the phenotype was more pronounced in the Tac1-/- and $\alpha \mathrm{CGRP}-/-\mathrm{DMM}$ 
mice in comparison with the WT DMM, verifying our previous observations in the 3Dreconstructed models.

The bone mineral density (BMD) of the meniscal ossicles were compared to the adjacent medial tibial epiphysis. Generally, the BMD of the ossicles was significantly lower in each group in comparison with the adjacent epiphysis (Figure 35). It was found that the BMD of the medial epiphysis was not influenced by the operation type (Sham vs. DMM) nor the absence of the targeted neuropeptides.
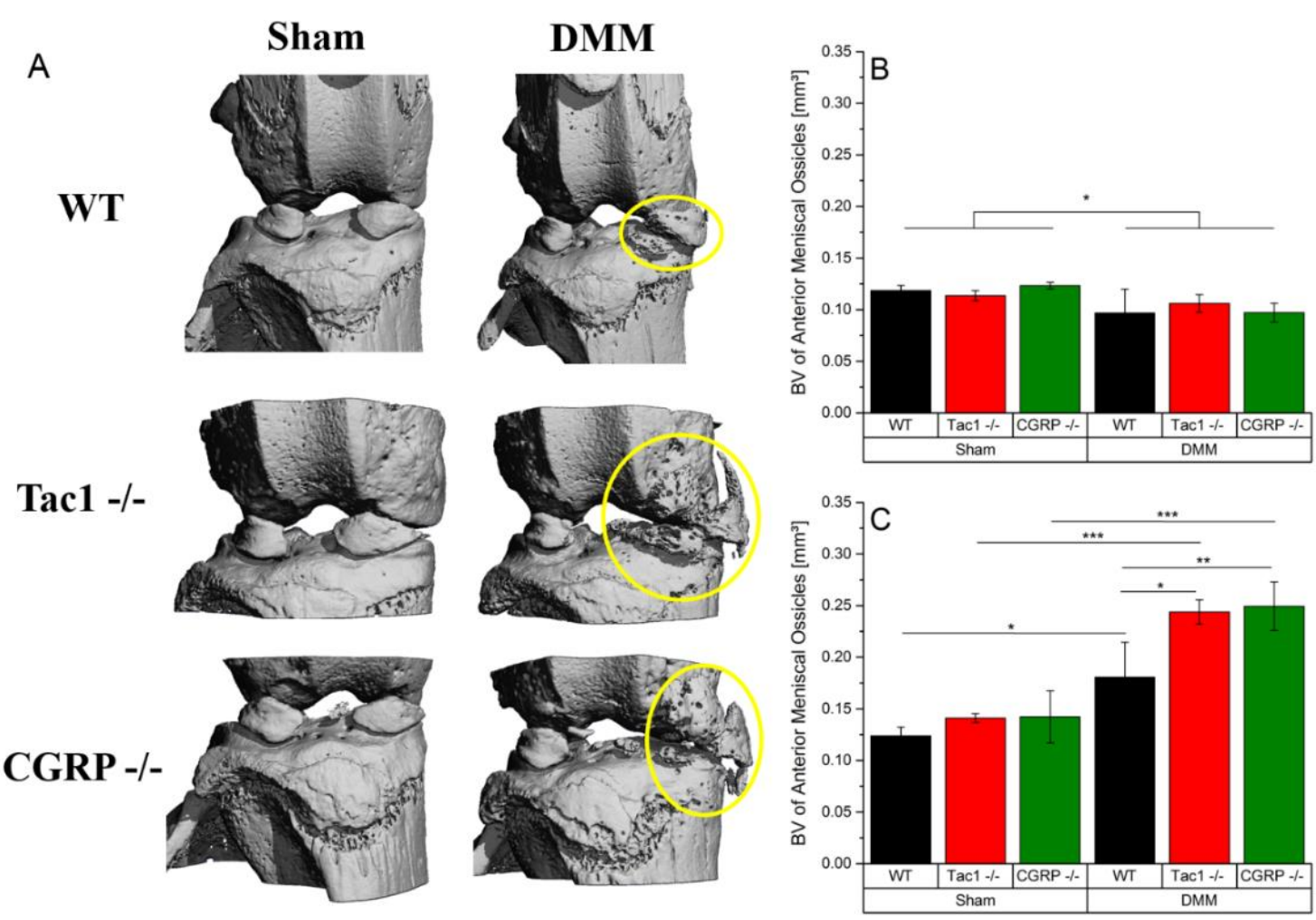

Figure 34. Meniscal ectopic bone formation after DMM surgery. (A) the 3D-reconstrcuted models on the knee joint at 8 weeks after the surgery shows the anterior meniscal ossicles, which was spatially elongated in the DMM mice (yellow ellipses). The volume of meniscal ossicles are quantified (B) 2 weeks, and (C) 8 weeks after the surgery and shows the dynamic of ectopic meniscal bone formation with time. Two-way ANOVA followed by Tukey post-hoc test was performed. ${ }^{*} p<0.05, * * p<0.01$, *** $p<0.001$. $\mathrm{n}=3$. 


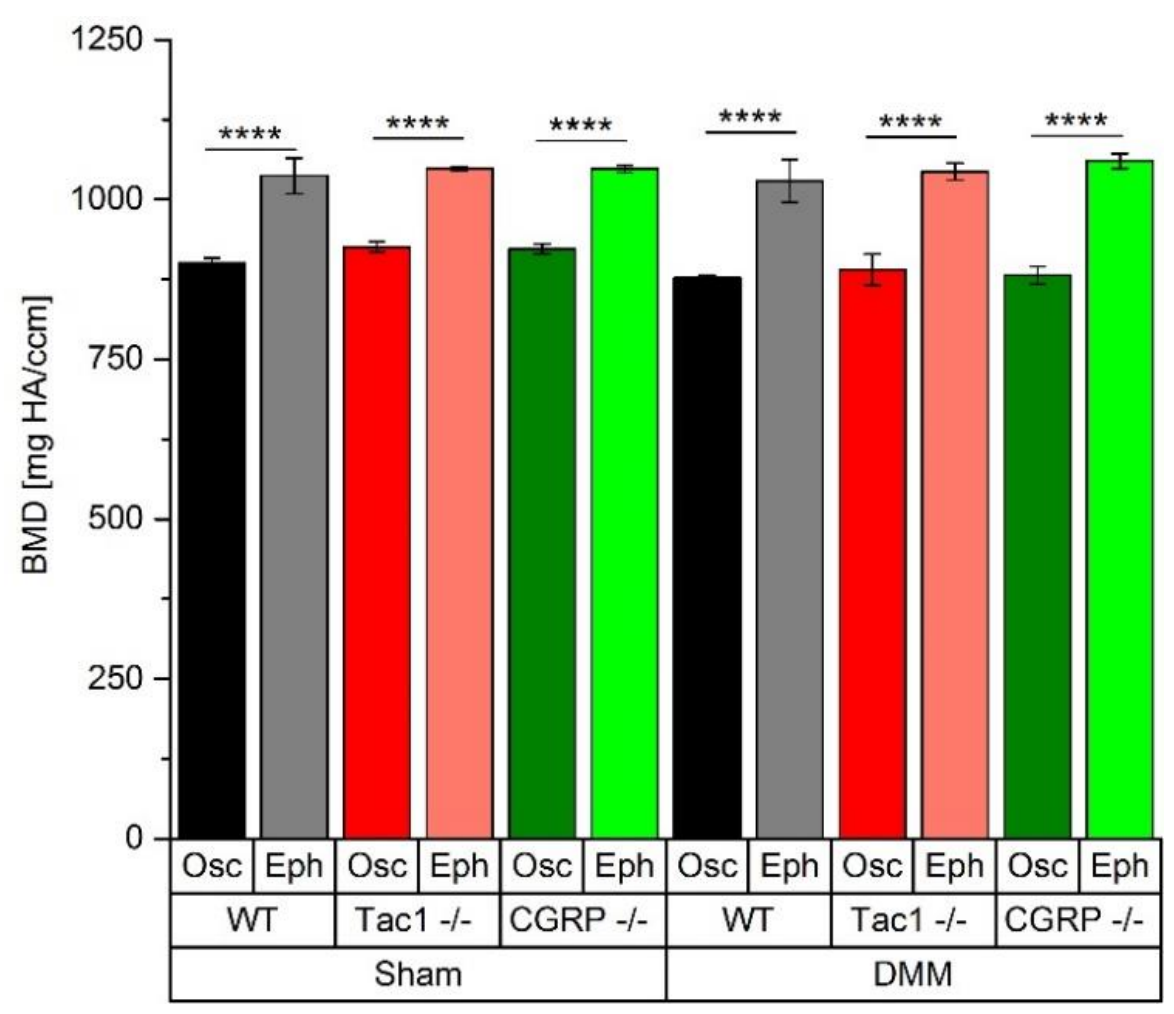

Figure 35. Comparison of the BMD in the medial tibial epiphysis and the meniscal ossicles. The BMD of the meniscal ossicles (Osc) and the adjacent medial epiphysis (Eph) was measured from the micro-CT imaging at 8 weeks after the DMM or Sham surgery. The statistical evaluation was conducted by Two-way ANOVA followed by Tukey post-hoc test. $* * * * p<0.0001 . \mathrm{n}=3$. 


\section{Discussion}

To unravel the role of SB (micro)architecture in joint physiology and pathophysiology during different stages and in different models, four main studies were conducted, and the results are presented in this dissertation: Namely, the maturation of the SB from young age to early adulthood was studied while introducing the SB microchannel system as an evasive anatomical microfeature that can be the focal point of further investigations. These microfeatures were studied in unprecedented detail in a physiological human model, as well as in idiopathic early OA. Based on these results, new hypotheses are proposed, and novel therapeutic strategies are presented. Finally, the early and late SB changes that are induced by traumatic $\mathrm{OA}$ are explored, while addressing the functionality of specific genes in physiological maintenance of the (subchondral) bone. In the following chapters, corresponding results for each study are consecutively discussed.

\subsection{Maturation-based changes of the subchondral bone}

The etiology of degenerative joint diseases has been the center of attention in OA research, particularly focusing on comparative analyses of different pathological stages of the joint (Kamibayashi et al. 1995; Bobinac et al. 2003; Yan et al. 2014; Chevrier et al. 2015; Huang et al. 2017). Nonetheless, studying maturation-based microstructural changes of the SB may provide fundamental principles to distinguish between different phases of bone development and pathological processes. In this regard, the SB microstructure and mineralization of the bovine femoral condyle was assessed by a combined micro-CT / histological method at a possibly interesting period of time between early-age and young adulthood. Different layers of the SB (i.e. CC, and SBP) were analyzed in small intervals from the tidemark, with the aim of detecting incremental changes. Furthermore, the high-resolution imaging allowed to three-dimensionally depict the heavily-disputed SB microchannel network for the first time, and qualitatively evaluate its morphological features.

It can be conceived that bovine subchondral bone may hold its own distinct characteristics compared to human. However, due to several reasons the bovine maturation model is comparable to that of humans. First, comparisons of genomic mappings between cows and humans have revealed that more than 80 percent of the analyzed cattle genes possessed human orthologs (Band et al. 2000), which means that bovine is genetically 
similar to humans; albeit less than rodents (Emes et al. 2003). Second, as inserted compressive loads on joint are major influences on bone configuration and microarchitecture (Ryan and Shaw 2013), the relevance of the bovine maturation model to that of human might be questioned given the 2.5-fold weight difference between 18 month-old cattle $(\sim 150 \mathrm{~kg})$ and 3-month-old calves ( $57 \mathrm{~kg}$; Fall et al. 1982; Mummed 2013). However, the bigger surface area of the tibial and femoral condyle in cows compared to human (Proffen et al. 2012), together with the fact that cows are quadrupeds mean that the net pressure on the knee joint might be comparable to humans, or at least the differences are less drastic than initially thought. Furthermore, in order to establish the correct association between various cattle and human age-groups, it is important to take into consideration the variations in lifespan as well. The natural lifespan of cattle is 15-23 years (Costagliola et al. 2016; Burgstaller and Brem 2017), while the natural lifespan of human beings is estimated to be 75-80 years (Dong et al. 2016). Using a linear regression model, it is approximated that 3-month-old calves and 18-month-old cattle are equivalent to 3-6 year-old preschoolers and 16-18 year-old young adults in human (Band et al. 2000; Burgstaller and Brem 2017). The estimated mean weight of 3-6 year-old preschoolers is $20 \mathrm{~kg}$ with a range of 17.3-23.0 kg (Vorwerg et al. 2013). 16-18 year-old young adults, on the other hand, have a reported weight range of 60.8-67 kg (Chiu et al. 2000; Diehr et al. 2008), meaning that the weight between the two age-groups is ca. $300 \%$ different. When we take into account the mean weight difference between 3-month-old calves and 18-month-old cattle (250\%), it can be inferred that maturation-based bovine model from young age to early adulthood is comparable to that of human in terms of weight/age-difference. Admittedly, since the sex hormones have a huge impact on bone development, the subject of sexual maturation is similarly important when the two models are being compared. Nonetheless, it is reported that the early age of puberty in male calves starts at 12-17 months of age as judged by the elevation of the Luteinizing hormone (Evans et al. 1995), which is in the same proximity to our early adult age-groups (18 months).

From our results, it was found that the thickness of the entire SB was increased with maturation, while the ratios of the calcified cartilage, and the subchondral bone plate thicknesses to the entire subchondral bone were relatively unchanged in the two agegroups (calf: $\mathrm{CC} / \mathrm{SB}=34.1 \%, \mathrm{SBP} / \mathrm{SB}=65.9 \%$; cattle: $\mathrm{CC} / \mathrm{SB}=30.5 \%, \mathrm{SBP} / \mathrm{SB}=$ $69.5 \%$ ), indicating that the increase of the entire SB thickness was most probably due to the natural growth of the animals. It has been reported that the mean CC of mature horses 
(15-19 years old) is more than $100 \mu \mathrm{m}$ thicker compared to young ( $<5$ years) healthy horses (Martinelli et al. 2002), which is similar to the observed CC thickness differences in bovine $(97 \mu \mathrm{m})$. It is known that changes in the SB thickness is crucial to distinguish different pathological stages of the joint (Burr and Gallant 2012; Li et al. 2013). In particular, sclerosis-induced thickening of the SB is a prominent hallmark of the endstage OA (Batiste et al. 2004; Buckland-Wright 2004; Intema et al. 2010; Wang et al. 2010). In end-stage models of osteoarthritis by destabilization of the medial meniscus (DMM), it was shown that SB of C77B2/6 mice became significantly thicker (Huang et al. 2017). Likewise, when bone extracts from the lesioned areas of human joints were analyzed, increased SB thickness was observed (Chevrier et al. 2015). As pure maturation and end-stage OA both lead to increased thickness of the SB, it is crucial to distinguish these different scenarios. On the other hand, if it is postulated that SB changes have a pathophysiological role in OA development and progression, the lower SB thickness in young models might be protective and might partially explain the low incidence of OA in the young population (Taheri et al. 2019).

An increased BV/TV for each individual layer of the SB was observed with maturation. Similar changes were observed in healthy Labrador Retrievers (Dingemanse et al. 2017), and DH guinea pigs (Yan et al. 2014). It was also found that the spacing between the trabeculae was significantly decreased with maturation $(62.5 \%, p=0.005)$, while a trend of increased Tb.Sp. was observed with distance from the tidemark (68.4\%); albeit insignificantly $(p=0.15)$. Hence, it can be concluded that "maturation" and "distance from the tidemark" affect the trabecular spacing inversely. Furthermore, the connectivity of the SB microstructure showed significant $(63 \%, p<0.05)$ and insignificant $(50 \%, p=$ 0.13) decline with distance from the tidemark and age, respectively. For the human femoral neck (Chen et al. 2010) and the subchondral bone of the proximal tibia (Chen et al. 2013), similar age-related findings are reported. The increase of bone volume fraction and the trabecular thickness combined with the less connectivity of the microstructure indicates a remodeling mechanism where the spacings and cavities are conjoined through the fusion of trabecular bone (Ding et al. 2003; Chiba et al. 2011).

As discussed in 1.2, few studies have explored the existence of subchondral bone perforations or defects that directly connect the basal cartilage layer to the deeper subarticular region (Holmdahl and Ingelmark 1950; Wilsman and Van Sickle 1972; Pan et al. 2009). Using a high-resolution micro-CT technique, the $3 \mathrm{D}$ configuration of the SB microporous structure was demonstrated for the first time (Figure 16). It was observed 
that the microchannels were abundant and relatively small in calves compared to the cattle. The total surface area of the microchannels was seemingly lower in mature animals, as indicated by the higher BV/TV of the subchondral bone in cattle. This may be significant if the microchannels play a nutritional or oxygen supply role as stated in other studies (Berry et al. 1986; Lyons et al. 2006). Indeed, it is hypothesized that the hyaline cartilage in an unossified chondroepiphysis (pre-maturation) is either nourished via intra-osseous vascular network that supply the chondrocytes and remove their waste products (vascular theory) or through the intercellular substance by a diffusion-based mechanism (diffusion theory), the latter being more widely accepted (Haraldsson 1959). Therefore, it is necessary to investigate healthy physiological models to assess if the presence of the SB microchannels - as possible nutrition pathways - is restricted to immature animals as stated elsewhere (Hodge and McKibbin 1969).

It was found that bone mineralization of the $\mathrm{SB}$ had a varying profile based on the distance from the tidemark. In particular, the increase of the BMD was striking in the 50-250 $\mu \mathrm{m}$ distance from the tidemark for both age-groups. Given the thickness of the individual layers of the SB (histomorphometrically measured in 3.1.1), it is reasonable to infer that this area is located entirely inside the CC layer. Hence, it is hypothesized that relative to deeper SB zones (e.g. SBP or subarticular region), CC is more dynamic and might play a significant role in bone growth and SB developmental transformation. Indeed, it is known that $\mathrm{CC}$ has an active role in bone remodelling via endochondral ossification (Lane and Bullough 1980). Similarly, articular cartilage degradation is partly due to a recurrent process in the calcified cartilage, where the tidemark progresses towards cartilage, and thus, should be considered as a major contributing factor in OA development (Anderson et al. 1993).

In cooperation with the Institute of Biomechanics, Technical University HamburgHarburg, the chemical composition of the SB was also evaluated using energy dispersive X-ray spectroscopy (results can be seen in (Taheri et al. 2019)). Interestingly, a depthdependent gradient of the chemical composition in the same area ascribed to the $\mathrm{CC}$ layer (uppermost $150 \mu \mathrm{m}$ of the SB) was found. Mean values of the calcium to phosphorus ratio $(\mathrm{Ca} / \mathrm{P})$ were ca. 1.59 for calves and 1.64 for cattle, which were comparable to reported data on young bovine trabecular bone (Kuhn et al. 2008). Interestingly, the ratio of $\mathrm{Ca} / \mathrm{P}$ was not different depending on the distance of the tidemark, even though both calcium and phosphorous exhibited depth-dependent alterations separately. Hence, the 
data demonstrates that calcium and phosphorous are co-dependent as previously reported in the literature (Lindsay et al. 2003; Shapiro and Heaney 2003; Bonjour 2011).

In conclusion, the maturation of healthy bovine subchondral bone is primarily dictated by an increase in BV/TV caused by trabeculae thickening. In comparison with the distance from the tidemark, maturation has less impact on the number (Tb.N.) and the connectivity of the trabeculae (Conn.D.). Calcified cartilage may be considered as a prime focus for future examinations owing to its steep mineralization profile and dynamic composition characteristics. Even though the SB microchannels are evasive anatomical peculiarities, they have an apparent age-dependent morphology, they provide a continuous interconnected pathway between medulla and articular cartilage, and might play a pivotal role in the (patho)physiology of the joint. Hence, the changes of the microchannel network in correlation with $\mathrm{AC}$ thickness and other hallmark features of OA are particularly interesting as focal points for further investigations. 


\subsection{Mapping of the SB microchannels in healthy human femurs}

Building on the previous observations regarding the SB microarchitecture, the methodology was expanded to describe the cartilage-bone marrow micro-connectors (CMMC) in a human physiological joint model. Different loading areas were determined by estimating the contact forces on the joint. A high-resolution profile of the CMMC was then created in adult intact femoral heads, where newly-defined indices were used to quantitatively describe the SB, and to detect load-dependent distribution pattern of the CMMC. Additionally, the results were correlated to the overlying cartilage, and new hypotheses were formulated based on the possible functionalities of the CMMC.

The 3D reconstruction of the osteochondral unit showed that the CMMC had diverse characteristics across the whole joint. The three-dimensional contiguity of AC, SB, and marrow spaces has so far been depicted only once, where serial H\&E-stained micrographs were overlaid (Lyons et al. 2006). Even so, the resolution was reportedly not sufficient to demonstrate the tidemark as a separate entity (Lyons et al. 2006). This might partially be due to the missing technology that is required to describe and quantify these elusive anatomical microfeatures, leaving a relatively uncharted realm in bone pathophysiology. Based on the visual inspections, it was postulated that local contact forces on the joint could influence the characteristics and the spatial configuration of these CMMCs.

In accordance with our hypothesis, it was found that the microchannels were strongly location-dependent and correlated with the estimated contact forces on the joint. Specifically, the number of the CMMC per $\mathrm{mm}^{2}$ was significantly higher in the loadbearing region (LBR) compared to the NBLR and the PR of the joint. Previous investigations conducted on healthy human femoral heads and tibial plateau point to a similar general pattern (Green et al. 1970; Lane et al. 1977; Meachim and Allibone 1984; Clark 1990; Milz S. and Putz 1994). A value of 6.25 per $\mathrm{mm}^{2}$ was reported for the vascular channels in the superior portion of the joint, while 4.69 vascular perforations were detected in the inferior part of the femoral head per square millimeter (Lane et al. 1977). The lower number of the vascular channels compared to our measured CMMC number in the LBR could be elucidated by two points. First, while it is confirmed that the SB perforations may contain capillaries and microvessels, it may be an infrequent occurrence associated with distinctive morphological features (Clark 1990). In other words, the CMMC number is not necessarily identical to the number of vascular channels within the CC layer (Green et al. 1970; Lane et al. 1977). Second, the measurement of the vascular 
channels by Lane et al was reported for the entire superior portion of the femoral head, meaning that the data were categorized into generally more stressed (the entire superior part) or less stressed (the entire inferior part) areas of the femoral head. On the other hand, we could define the load-bearing region of the joint in a much more confined manner using a gait analysis approach. This signified that unlike Lane et al, we did not pool the vascular channels at the peripheral rim of the joint with those at the load-bearing region.

As a matter of fact, the definition of what constitutes a load-bearing area of the femoral head is not unanimous in the literature. In one study, the angle between the two lines connecting the medial and lateral edges of the lunate surface and the center of the femoral head has been defined as the load-bearing region, while the less weight-bearing area was characterized by the lines connecting the center of the femoral head and the lower margin of the fovea capitis (Teshima et al. 1999). The peripheral rim located at the superomedial portion of the joint was accurately differentiated for data categorization in another study. However, at the inferomedial part no such distinction was made, and the data were pooled with the corresponding non-contact area of the inferomedial part (Woods et al. 1970). Due to different definitions of the load-bearing region, as well as the strong variability of the CMMCs based on different locations on the joint, we opted for a gait analysis approach to classify various loading areas. The estimated contact forces were highly concentrated superolaterally to the intersection between the long axis of the femoral head and the surface of the joint, and were slightly inclined anteriorly (Figure 17A). The direction of hip contact force has been measured in another study using instrumented hip implants, and was found to be consistent with our results (Bergmann et al. 2001).

It was also found that the maximum (Feret) and the minimum diameter (MinFeret) of the SB microchannel network were varied based on the location on the femoral head. The smallest CMMC were generally observed at the LBR, while the channels were progressively enlarged at the NLBR and the outermost rim of the joint. Another study on the adult human tibial plateau reported a similar general pattern (Milz S. and Putz 1994), with a ca. 10-12\% difference in the calculated mean sizes, which can be explained by differences in technique (Clark 1990) as well as the size-based classification of the channels by Milz and Putz, compared to our region-based categorization (Milz S. and Putz 1994). An SEM-based study of the cartilage-bone interface in human distal femurs showed that the articular and calcified cartilage are interlocked by "protrusion-pores" in the range of $1.98-114.2 \mu \mathrm{m}$, and with a mean size of $17.0 \mu \mathrm{m}$, while at the interface of CC and subchondral bone plate the mean diameter was $34.1 \mu \mathrm{m}$ (Liu et al. 2011). 
Nevertheless, the pores below $7 \mu \mathrm{m}$ are mainly craters, which are considered to be a typical calcification feature that often occurs around chondrocytes (Duncan et al. 1987). They appear as discontinuous "bubbles" in the 3D-data sets, do not penetrate the SB, and thus were distinguished from the CMMC. Pooling these protrusion pores or bubbles with the CMMC would obviously result in an underestimation of the CMMC's mean size.

There have been several qualitative terms to describe and categorize the morphology of the SB perforations. For example, the small, interconnected microchannels that are frequently observed in the weight-bearing region of the joint has been referred to as dendritic canals (Holmdahl and Ingelmark 1950), type A defects (Woods et al. 1970), and finger-like canals or branches (Clark 1990; Milz S. and Putz 1994; Hwang et al. 2008). Similarly, what is seen in this study as a typical large microchannel at the outermost peripheral rim of the joint has been previously cited as "wide, ampulla-like contacts" (Holmdahl and Ingelmark 1950), "irregularly-shaped focal contacts" (Clark 1990), "large, void spaces" (Hwang et al. 2008), or "hole-like gap formation" (Milz S. and Putz 1994). Since these terminologies are abstract, qualitative, and might be subject to many interpretations, a more useful solution to illustrate region-based morphological variations of the CMMC is quantification-based approaches. In this regard, the morphology of the SB microchannel network was quantified by measuring the circularity index. It was found that the microchannels had a generally round and circular morphology in the LBR, while the Circ. was increasingly reduced at the outermost rim of the joint. The steep decline of the Circ. profile at deeper distances of the tidemark (> $150 \mu \mathrm{m}$; Figure 23A) expressed the integration of the microchannels at the interface of the cortical end-plate and subarticular region. At even deeper distances below the end-plate, Circ. reflected the irregularity of spacings between the subchondral trabecular bone.

A recurrent dichotomy in literature can be clarified by the 3D imaging-histology juxtaposition (Figure 20). As CMMCs are not completely perpendicular to the tidemark, a microchannel that is fully connected to the cartilage is hardly exposed by a vertical plane of sectioning. Hence, most CMMCs appear to be incomplete in micrographs or histological slides (Woods et al. 1970). They are often partially cut by any plane of sectioning, and usually appear as isolated virtual islands (Lyons et al. 2006). This can explain why photomicrographical and histological-based investigations of adult models have asserted that subchondral bone channels do not often cross the CC layer (Hodge and McKibbin 1969; Clark and Huber 1990). On the other hand, several perforations were found to transverse the calcified cartilage layer, when the AC-CC interface was explored 
superiorly (Lane et al. 1977). Indeed, it is clearly demonstrated that while some microchannels circumvent the $\mathrm{CC}$ layer at the point of cartilage contiguity, others invade this layer and forge through it to reach the articular cartilage (Figure 21C). Hence, it is proposed that future histology- or immunohistochemistry-based studies on the CMMC would be more instructive if the slides are embedded and prepared superiorly, i.e. parallel to the SB's superficial surface.

It was also found that the $\mathrm{AC}$ was occasionally extended beyond the tidemark, interlocked with the CC layer, and adjoined the subchondral end-plate or marrow spaces. These AC prolongations have been observed in another study, where they were often accompanied by the tidemark (Lyons et al. 2006). Furthermore, we could show the presence of microvessels within some of the channels in healthy femoral heads, and indicated by a positive vWF expression of the CMMC's content. vWF is a large glycoprotein that is produced constitutively in endothelium, as acts as a negative modulator of angiogenesis (Starke et al. 2011; Rauch et al. 2013), and thus, can be considered a health biomarker in the context of OA.

The measurement of AC and SB thicknesses over the whole femoral head revealed the interdependent nature of the bone-cartilage unit. An evident gradient to the thickness distributions of both tissues was found, which was correlated to the load-bearing areas of the joint. The thickest cartilage and SB were observed in the LBR, while thin layers of AC and SB were found in the PR (Figure 18). Similar observations are reported for other human joints (Woods et al. 1970; Clark 1990; Milz S. and Putz 1994). Eckstein et al. generated a density map of the human patella, and interpreted the overlap of cartilage and SB thickness distributions as an expression of the biomechanical function in the joint (Eckstein et al. 1992). Likewise, it was observed that thicker AC was accompanied by thicker SB in human acetabula (McGibbon et al. 1998). In the current study, we also found a significant moderate positive correlation between $\mathrm{AC}$ and $\mathrm{SB}$ thicknesses $(\mathrm{r}=$ $0.48 ; \mathrm{p}<1 \mathrm{e}-13$ ) for the human femoral head, supporting these results.

Several functional interpretations are given for the microchannel systems of the SB, even though their precise functions are still unknown. The new results presented in this study can extend the current knowledge regarding the SB microchannel network, and shed light on their functionality. Hence, possible functions of the cartilage-bone marrow microchannels will be discussed and interpreted in the context of this dissertation. 


\subsubsection{Extended possible functions of the CMMC based on the new results}

The traditional view has been that the nutrition of adult cartilage depends almost entirely on the transsynovial route, assuming calcified cartilage to be an effectively impermeable barrier (Collins 1949; Mankin 1963; Stockwell and Barnett 1964; Maroudas 1968; Maroudas et al. 1968; Wang et al. 2013). Even though subchondral perforations were detected in the early works on adolescent rabbits, the lack of such observations in mature models led some scientists to believe that subchondral-driven nutrition of the articular cartilage is only a feature of immature animals (Hodge and McKibbin 1969; Mital and Millington 1970). Since then, and as discussed in detail, a growing body of evidence has shown the presence of the subchondral bone perforations in a variety of older human joints, which highlights inter-species differences and casts doubt on the relevance of animal studies to human. It is now hypothesized that nutrition from the marrow cavity through the SB plays a critical role in cartilage homeostasis and functionality (Greenwald and Haynes 1969; Meachim and Allibone 1984; Milz S. and Putz 1994; Lyons et al. 2006; Schilling 2017).

There are several rationales behind the nutritive functional theory of the SB perforations: (1) Adjacent to the subchondral end-plate, a surprisingly high number of arterial and venous vessels can be found that branch out to the vicinity of the calcified cartilage (Björkström and Goldie 1980; Imhof et al. 1997). Often, the highly vascularized areas of the human body are associated with excessive functional activities that necessitate a high regional nutrition capacity (Imhof et al. 1997). It is reported that the blood flow rate in the subchondral end-plate region is three to ten times higher than that in the trabecular bone (Nakano et al. 1986; Imhof et al. 2000), which might be reflective of the high local stress on the joint surface, as well as its high metabolic activity. The abundance of the microchannels in the load-bearing region of the joint, as measured in this study, can then be interpreted as a functional adaptation for providing adequate nutritive support (Milz S. and Putz 1994).

(2) It is frequently reported that in normal non-arthritic joints, the areas of the higher AC thickness and SB thickness are colocalized and correlated with the load-bearing regions on the joint (Woods et al. 1970; Clark 1990; Eckstein et al. 1992; Milz S. and Putz 1994; McGibbon et al. 1998). We have also found a significant moderate positive association between the AC and SB thicknesses for the human femoral head, which was correlated with the contact force mapping on the joint, and interpreted as an expression of the 
biomechanical function in the joint. Thus, the high load-bearing areas of the joint, not only have the highest local density of SB perforations, but also the thickest overlying articular cartilage. In such regions, the length of the diffusion pathway from the subchondral region to the basal cartilage layer is shorter than that from the cartilage surface (Imhof et al. 1997), and it is conceivable that the SB perforations provide nutrition to the deeper chondrocytes adjacent to the tidemark (Duncan et al. 1987). Moreover, Curtis et al. demonstrated connective vascular pathways between different osteocytes of adjacent lamellar systems in the cortex of long bones and identified a "critical distance" to the next blood vessel, which could not be exceeded for an adequate supply over longer distances (Curtis et al. 1985). According to Ham (Ham 1952), effective supply should only be possible up to a distance of 100-200 $\mu \mathrm{m}$, while Lozupone et al. consider distance values of 230-250 $\mu \mathrm{m}$ for the proximal tibial cancellous bone, but a mean distance of about $250 \mu \mathrm{m}$ is typically agreed upon (Lozupone and Favia 1990). Based on these ideas, it can be postulated that the supply of the thick parts of the subchondral bone depends on the presence of a microporous network, which can explain why the perforations are predominantly found in such regions. Interestingly, I have even identified intermittent occurrence of the microchannels in some trabeculae, which is confirmed in another report (Milz S. and Putz 1994). These channel-containing trabeculae are often slightly thicker than the solid trabeculae without a perforation system, and their diameter is usually between 230 and $400 \mu \mathrm{m}$. Hence, the notion that the microchannels maintain a critical distance for the effective diffusion is applicable in interpreting the presence of channellike cavities in the trabecular bone as well.

(3) It has been shown that there is a direct correlation between the blood supply and the extravascular fluid transport in bone tissue (Fritton and Weinbaum 2009). The movement of the extravascular fluid allows nutritive substances to reach to the cells that are located within the bone. Additionally, the interchange of pore fluid between the vascular and the lacunar-canalicular porosity within the bone has been deemed crucial in nutrient transport, and is often attributed to recurrent mechanical loading and blood pressure shifts (Cowin und Cardoso 2015). Hence, it is plausible that the observed microchannels are passages for the interstitial fluid movement between the cartilage and the medullary cavity. It is found that the pressure of deeper layers of the articular cartilage can be relayed via fluid shifts (Ateshian 2009; Occhetta et al. 2019). The effect of such pressure fluctuations could, on the one hand, influence the extent and type of nutrient supply, and on the other hand, the formative stimulus on the bone cells in the basal layers of the 
articular cartilage (Milz S. and Putz 1994; Stavenschi et al. 2018). Supporting evidence has shown that osteocytes that were located at depths of up to five lamellae could be reached and nourished via their canaliculi when pressure-induced fluid shifts existed (Kufahl and Saha 1990). It was further hypothesized that the lack of such fluid shifts could be a cause of immobilization-induced pathological changes of the bone. Assuming that the microchannels are passages for liquid transfer from the medullary cavities to the articular cartilage (or vice versa), the observation that the smaller-sized microchannels $(15-30 \mu \mathrm{m})$ are located in areas of the joint that are subjected to the highest mechanical strain has been found to be surprising (Holmdahl and Ingelmark 1950). However, the strong compressive pressure on the SB in load-bearing regions is in favor of the liquid transit through these even tiny microchannels. On the other hand, at the peripheral and non-load-bearing areas, larger but less frequent perforations are exposed to less mechanical pressure. Hence, the microarchitecture of the subchondral bone seems to be adapted to sustain an adequate profile of fluid shifts in relation to the thickness of the overlying cartilage.

According to another theory, the presence of SB perforations in old populations are attributed to pathological processes (Green et al. 1970; Milgram 1983; Oettmeier et al. 1989). The age-related reduction of subchondral vessels in normal human femoral heads has been interpreted as an early degeneration marker (Woods et al. 1970), while direct contact of capillaries with the basal cartilage of adult joints has been considered to be a part of a remodelling process (Ogata et al. 1978; Clark 1990). Indeed, vascular invasion and angiogenesis at the osteochondral junction are indispensable for OA progression (Bonnet and Walsh 2005; Suri et al. 2007; Burr and Gallant 2012). However, angiogenesis refers to the advancement and invasion of new blood vessels into the cartilage, and thus implies a change over a period of time. Part of the conflict might arise from a lack of distinction between vascular structures in normal, adult joints and the newly-formed capillaries that are known to play a crucial role in subchondral remodeling. It is reported that in intact joints, most of the capillaries in the subchondral bone reside in the adjacency of the calcified cartilage, and the vessels that literally touch the lower margin of the articular cartilage are relatively few, with no evidence that they form loops (Clark 1990). This type of blind vessel penetration casts doubt on the capacity of an "end vessel" for nutrient transportation (Clark 1990). Nevertheless, the limited number of capillaries that penetrate healthy cartilage does not necessarily marginalize the nutritive functional theory of SB perforations, since a diffusive transport based on extravascular 
fluid is postulated and not a blood-borne mechanism. Indeed, several studies have pointed out that the porosity of the subchondral bone is not equal to the vascularity of the calcified cartilage (Green et al. 1970; Lane et al. 1977). Hence, it is conceivable that the vascularity in the microchannel network of the healthy subchondral bone, and the invasion of new blood vessels that occurs later in pathological processes are not mutually exclusive, but rather representative of different health-stages of the joint over time.

It has been confirmed that diffusion via the end-plates of intervertebral discs is the primary pathway for nutrition in adolescent rabbits (Brodin 1954). It is even asserted that more than $50 \%$ of the oxygen, glucose, and fluid requisites of articular cartilage can be supplied by the perfusion of subchondral vascular plexus (Imhof et al. 2000). In order to test the hypothesis that both nutritional pathways from the SB and the synovial fluid play a significant role in homeostasis and integrity of cartilage, the nutrition of the distal femur of 95-month-old male rabbits was cut off by inserting a Polyvinyl Chloride cap in the trochlea. The authors concluded that that synovial fluid-derived nutrition was the main nutritional source for the functionality and maintenance of adult cartilage structure (Wang et al. 2013). On the other hand, studies on adult male baboons shows that the long term (> 3 years) obstruction of the contact between the vascularized SB and AC leads to degenerative changes compatible with OA (Malinin and Ouellette 2000). It was asserted that if the experiments were concluded at one year or sooner, the aforementioned degenerative alterations would not have been evident. Therefore, it seems that both possible mechanisms of nutrition (transsynovial and subchondral) might operate independently, and each may play a significant role in maintaining the integrity and functionality of the articular cartilage (Duncan et al. 1987; Schilling 2017). 


\subsection{SB microchannels in early osteoarthritis}

In human studies, there is a lack of longitudinal data on SB alterations from the early pathological onset of the disease to the end-stage clinical OA. In particular, since very early changes occur well before the disease is diagnosable in clinical settings, they cannot readily be investigated in humans. Furthermore, current non-invasive analytical methods for the determination of SB changes have low sensitivity and are therefore inadequate to quantify minor changes (Mastbergen and Lafeber 2011). Here, our established methodology based on high-resolution imaging was applied to describe the SB microarchitecture in early OA human femoral heads. It was hypothesized that the SB microarchitecture (in particular, the cartilage-bone marrow microchannel connectors) undergo early structural changes in OA, and that these changes can be utilized for the development of novel therapeutic strategies and early diagnostic tools.

By superimposing the areas of early cartilage degeneration and partial thickness chondral defects in five femoral heads, a map of the most prevalent early OA regions was obtained. It was observed that early chondral defects were predominantly found at the inferomedial and posterior portion of the femoral head, associated with the NLBR and the peripheral rim of the joint. Less than $10 \%$ of all the extracted measuring points were located at the LBR, which might be surprising considering the fact that increased chronic loads are major contributors to cartilage damage and degeneration (Goldring and Goldring 2010; Roemhildt et al. 2010). Nonetheless, it has been reported that early cartilage fibrillation and splitting is more commonly seen at the edges of the joint contact regions or at the inferomedial portion of the human femoral head, even though SB exposure in end-stage OA patients usually develops on or near the central, load-bearing regions (Meachim and Emery 1973; Meachim et al. 1980; Meachim and Allibone 1984; Burr and Gallant 2012). This paradox has led some scientists to believe that focal chondral defects have a small capacity on the inferomedial portion to progress into osteoarthritic SB exposure, while those located superocentrally, have a higher osteoarthritic potential, even though they are less frequent (Byers et al. 1970). In our measurements, the thickness of the AC was not significantly different compared to the healthy groups (except at the rim of the joint), even though a clear diminishing trend could be observed. Moreover, small, focal chondral lesions often do not result in joint space narrowing and hence, may be present in knees that appear to be normal upon radiographical inspections (Guermazi et al. 2012; Hayashi et al. 2014). It is, therefore, equivocal whether focal partial-thickness chondral lesions are as relevant in the context of OA progression as full-thickness defects. Nevertheless, it has 
been demonstrated that regardless of defect depth, prevalent focal cartilage defects in an isolated sub-region in the tibiofemoral joint increased the risk for development of new chondral defects in unaffected sub-regions of the same tibiofemoral joint (Guermazi et al. 2017). Hence, local investigation of partial-thickness chondral lesions and its underlying SB is important when exploring preventive measures or repair strategies of degenerative changes (Jansen et al. 2008).

A reduction of the SB thickness in early OA was observed, which was specifically significant at the NLBR and the PR of the joint. Interestingly, this phenomenon appeared as a localized occurrence linked with the most prevalent areas of early cartilage degeneration. In contrast to hallmark features of late-stage OA such as subchondral bone plate thickening (Chen et al. 2017), the early phase of OA is generally associated with the structural deterioration, and an early loss of bone due to elevated bone remodelling (Bettica et al. 2002; Burr and Gallant 2012). In particular, thinning of the subchondral plate has been reported in early OA animal models (Batiste et al. 2004; Meyer et al. 2008). In two different canine models of OA, subchondral plate showed obvious propensity for thinning as an early response, which was interestingly independent of loading conditions (Sniekers et al. 2008). Likewise, the colocalization of subchondral plate thinning with cartilage degradation has been reported elsewhere (Bellido et al. 2010; Intema et al. 2010).

Here, I presented the first report regarding the changes of the SB microchannel network in early OA. In general, the CMMC maintained the same location-dependent characteristics that was previously observed for the healthy femoral heads; i.e. the LBR was perfused with abundant small microchannels, whereas large, intermittent, and elongated perforations were found at the NLBR, and in particular, the peripheral rim of the femoral head. Compared to the healthy samples, however, a marked increase of the maximum and minimum CMMC size was detected in early OA samples. At the NLBR and the PR, the significance level was particularly high, and more pronounced for the Feret than the MinFeret. Given that the CMMC number was unchanged compared to the healthy groups, it can be inferred that the porosity of the SB was increased in early OA. This is in accordance with a handful of studies that confirmed elevation of the subchondral plate porosity during early stages of OA development (Hwang et al. 2008; Intema et al. 2010). In an instability-induced OA mice model, it was detected that only two weeks after intraarticular injection of collagenase, osteoclast activity was significantly increased directly below the subchondral bone plate, creating a large 
increase in the number and volume of the subchondral bone plate porosity (Botter et al. 2011). Hence, the increase in the size of the SB perforations can be interpreted as an adaptive mechanism to enhance cartilage-bone crosstalk for diffusion of small molecules (Lyons et al. 2006; Hwang et al. 2008; Pan et al. 2009; Pan et al. 2012).

All lines of evidence point to an increased bone remodelling in the early stages of OA, and even though its fundamental mechanism is not completely understood, factors such as angiogenic-induced vascularity and microfracture repair have been proposed (Verborgt et al. 2000; Luyten et al. 2006). In particular, microfractures are believed to be a nidus for the initiation of elevated osteoclastic activity that occurs in association with the process of repair (Burr and Gallant 2012). This induces early bone resorption and might serve to enlarge any site of microdamage (Duncan et al. 1987). Based on these findings, it can be postulated that the CMMC are local initiators of targeted remodelling that undergo a significant size enlargement in early OA.

Considering this line of thought, the paradox that early cartilage degeneration is more prevalent at the non-load-bearing areas and outermost rim of the joint can be explained by the following mechanism: At the margins and peripheral areas of the joint: (1) The subchondral bone plate has less density compared to the weight-bearing regions (Burr and Gallant 2012). (2) The shear stress concentration is high, which is shown to be the pivotal biomechanical cause of cartilage matrix destruction (Thambyah and Broom). And (3) as seen in 3.2.5, the size of the CMMC is relatively large. Consequently, a model for early OA development is conceivable in which the size of the SB microchannels is further increased, leading to a highly-porous SB and less mechanical support (specifically) against shear stresses at the edges of the joint. This would eventually result in early cartilage fibrillation and degradation. It is suggested that the SB perforations might become sites for early formation of cysts, such as seen in osteoarthritis (Duncan et al. 1987). Diminished support of the compressed cartilage might then favor entrapment of fluid in the weaker areas, while the cartilage itself might bulge into the indentation. Other evidence illustrated that the formation of cysts and early cartilage fibrillation frequently occurred beneath the meniscus of human tibial plateau (Duncan et al. 1987). Interestingly, this site has a high frequency of larger perforations supporting our interpretation.

The early subchondral thinning and deterioration is seemingly in contrast with hallmark features of OA such as SB sclerosis and subchondral bone plate thickening. However, it is proposed that repetitive occurrence of microfractures within the subchondral bone plate 
and/or the subarticular trabeculae, can shift the balance in favor of an osteoblastic repair mechanism. This would lead to excessive matrix productions, and ultimately the fusion of adjacent trabeculae into sclerotic bone (Christensen et al. 1982; Duncan et al. 1987; Ding 2010; Gianakos et al. 2020). Hence, indisputable signs of OA such as subchondral sclerosis and the thickening of the cortical end-plate, may only be observed as late-stage events during the pathological cycle (Batiste et al. 2004; Intema et al. 2010; Wang et al. 2010). A late-stage remodelling sequence would then be plausible in which the subchondral densification acts as an occluding phenomenon for the CMMC-driven diffusion; i.e. the formation of excessive bone matrix can close off the connective pathways between the medullary cavity and the basal layer of the cartilage, which would impair the delivery of nutrition to the deep-seated chondrocytes (Duncan et al. 1987). Indeed, it was found that the previously-enlarged perforations that were developed during early stages of OA (two weeks after OA induction), disappeared after 4 weeks, which coincided with a significant elevation in osteoblast activity in the subchondral trabecular bone (Botter et al. 2011). A model for the joint degradation in late-stage OA is proposed in which the metabolism of the chondrocytes is sufficiently altered after the obstruction of diffusive pathways. This would allow for chondrocytes located along the tidemark to develop a hypertrophic phenotype, a local perichondrocytic calcification, and an ensuing elevation of alkaline phosphatase activity (Schumacher 1996; Niino-Nanke et al. 1998; Pesesse et al. 2011). This series of events could further explain the advancement and duplication of the tidemark, which contributes to articular cartilage thinning and deterioration (Duncan et al. 1987; Goldring 2009).

\subsubsection{CMMC-based therapeutic strategies}

It was demonstrated that (micro)structural changes such as SBP thinning and CMMC widening occur in subchondral bone, well before major alterations in cartilage thickness. This reaffirms the notion that SB changes are not merely an effect of cartilage degradation, but may directly cause or trigger pathological processes such as OA. Many of today's OA treatment strategies are applied at late pathological stages, which can restrict the intended disease-modifying effects. Hence, it is proposed that structuremodifying therapies should be implemented early in the course of the disease (Mastbergen and Lafeber 2011).

Microfracture is a marrow-stimulating procedure for AC repair in which several perforations or microfractures are made into the subchondral bone plate (Seo et al. 2011). 
Marrow components such as growth factors and other healing proteins can then reach to the defect region, and from a surgically-induced super clot. The super clot provides an enriched environment for the marrow mesenchymal stem cells to differentiate into chondrocytes (Steadman et al. 2002). Microfracture has been associated with poor clinical outcome due to some disadvantages. Namely, the mesenchymal stem cells differentiation leads to the formation of fibrocartilage, which is mainly composed of type I collagen and contains less proteoglycans compared to hyaline cartilage (Seo et al. 2011). Thus, fibrocartilage is biomechanically inferior to hyaline cartilage, leading to collapse under repeated mechanical stress (Shah et al. 2007).

Nevertheless, the basic principle of microfracture technique is the (re)introduction of SBdriven diffusion pathways for cartilage repair. As we demonstrated, these connective pathways are already present in the normal joint (CMMC); albeit with drastically different characteristics compared to the osteochondral lesions in microfracture. For example, a critical defect area of $150 \mathrm{~mm}^{2}$ (equivalent to a cylinder with a diameter of $14.00 \mathrm{~mm}$ ) has been proposed in order to maintain the integrity of the subchondral bone plate during microfracturing (Choi et al. 2009). This value is ca. 100 times higher compared to the largest CMMC observed in the healthy femoral heads. Moreover, the perforations in microfracture are approximately 2-4 mm apart (Steadman et al. 2001), whereas a much higher CMMC density (5-10 per $\mathrm{mm}^{2}$ ) has been found in healthy SB. Based on our findings, we invented a revised treatment strategy for re-establishing SB microchannels in pathological joint that mimics the healthy SB micro-characteristics. The invention addresses aspects of modifying SB microarchitecture such that the CMMC size, number, and their distribution varies dependent on, and is adapted to, the LBR, NLBR, and PR of the subchondral bone, thereby allowing for a particularly realistic imitation of healthy SB microarchitecture. Briefly, the procedure consists of removal of the uppermost part of the pathological SB by microsurgery techniques, which would expose a cross-sectional area of the SB with increased gross porosity (Figure 36). This should theoretically provide sufficient access to the underlying bone marrow and vascular structures. Depending on preferred CMMC structure in different loading areas of the joint, appropriate membranes are selected by the surgeon, and are surgically implanted superiorly on the pre-treated SB. This ultimately re-introduces the physiological number of continuous openings from the upper surface of the SB to the underlying trabecular bone spacings. The membrane is fixed on the SB by fibrinogen glue and suturing as necessary. 


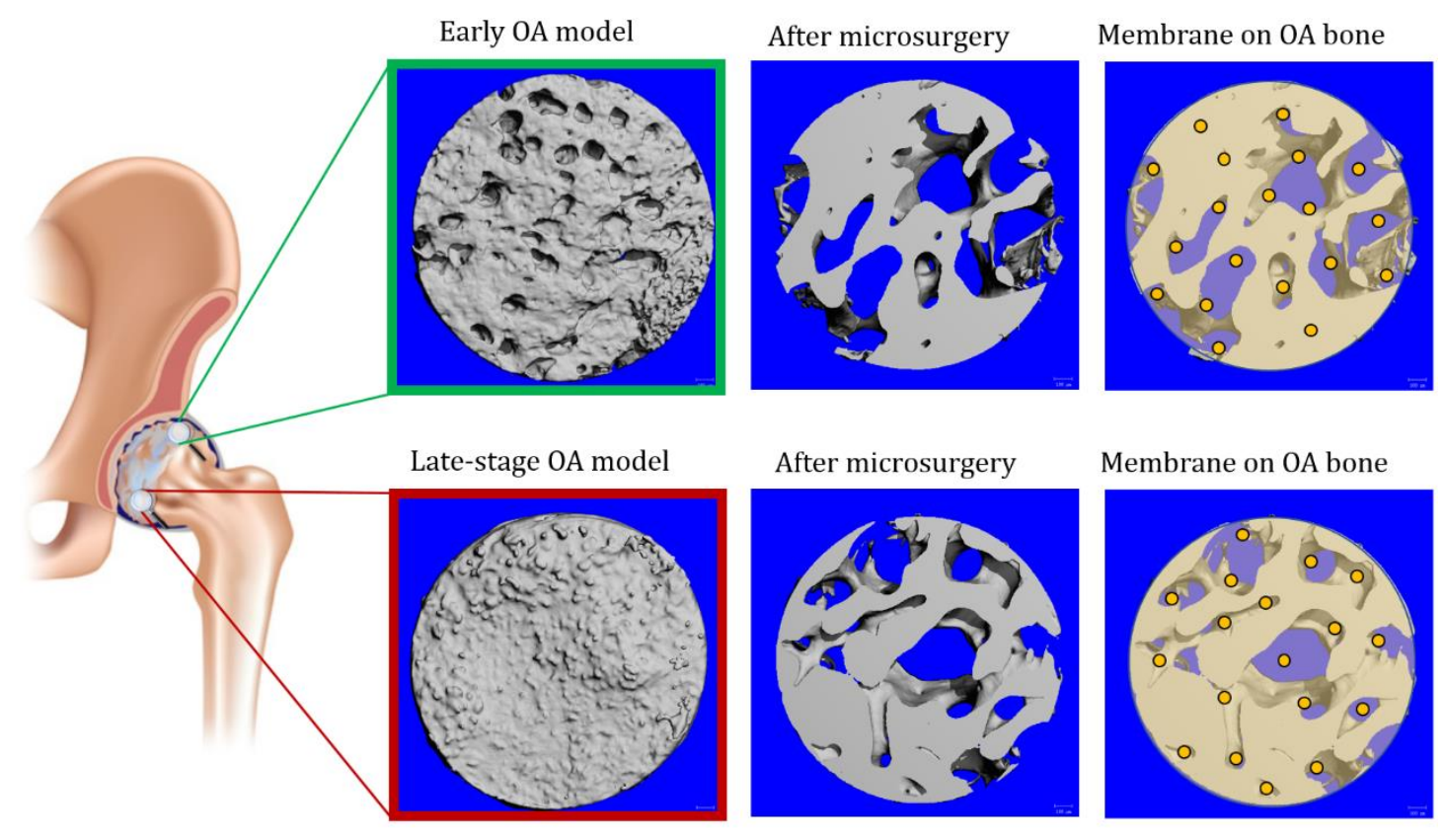

Figure 36. A schematic representation of the new CMMC-based therapeutic strategy for cartilage repair. Two pathological models with altered CMMC characteristics are postulated: the elevated porosity model in early OA, and the sclerotic late-stage model with presumed "closed-off" SB. The uppermost part of the SB including the CC layer is removed by microsurgical techniques such as curette-assisted debridement. This would reveal a cross-sectional portion of the SB that contains a relatively high porosity ratio. Based on the location on the joint in terms of loading areas, membranes with preferred CMMC characteristics are selected by surgeon, implanted on top of the pre-treated SB, and fixed by fibrinogen glue and suturing. The procedure is based on a recently filed US patent with the University Medical Center Göttingen entitled "microchannels in subchondral bone and membranes comprising same for the treatment of osteoarthritis" 


\subsection{Early and late subchondral bone changes in a traumatic OA model}

Even though OA is a multifactorial disease that involves the interaction between multiple joint tissues, the mechanical environment of the joint in the course of body movement has a substantial effect on the initiation and development of OA. In this study, a traumatic OA model of surgical destabilization of the medial meniscus (DMM) was implemented to induce excessive mechanical loading on the articular cartilage and the subchondral bone. The OA-induced early and late SB changes were then examined, while highlighting the effect of sensory neuropeptides substance P (SP) and alpha-calcitonin gene-related peptide ( $\alpha \mathrm{CGRP})$ on the subchondral bone microstructure.

8 weeks after the OA induction, an increase in the medial CC thickness was visible, which occurred in all three groups (i.e. WT, Tac1-/-, and $\alpha$ CGRP $-/-$ ). The heatmaps of the CC layer, showed that the increase was mostly localized at the load-bearing regions (i.e. contact points) of the medial and lateral condyle, as well the newly-formed CC overlying the osteophyte formations. It is reported that the CC goes through structural alterations when there is an instability of mechanical loading on weight-bearing joints (Burr and Radin 2003). Furthermore, a noticeable proteoglycan loss was detected in the medial tibial plateau, as early as two weeks after the OA induction. In an anterior cruciate ligament transection (ACLT) osteoarthritis mouse model, it was demonstrated that the CC thickness was increased, while the tidemark moved closer towards the articular surface. Similar to our findings, a significant trauma-induced proteoglycan loss was detected at the calcified cartilage zone adjacent to the subchondral plate (Zhen et al. 2013). An increased body of evidence points to the fact that cartilage calcification plays a pathogenic role in OA, and occurs as an active process (Ea et al. 2011). It was even shown that the injection of calcium pyrophosphate microcrystals into the meniscectomized rabbit knee joints exacerbated the cartilage degradation (Fam et al. 1995).

Interestingly, trauma-induced calcification was also observed in the meniscus. At the medial side of the knee joint where OA induction occurred, heterotopic ossification (HO) was observed as a distinct phenotype, which was more pronounced in both neuropeptidedeficient DMM mice. It has been proposed that the calcification of the meniscus changes the biomechanics of the whole joint and contributes to cartilage fibrillation and fissures (Cheung et al. 2006; Ea et al. 2011). In a spontaneous OA model, it was observed that 
meniscus calcification occurred even before cartilage lesions, while treatments with phosphocitrate - a powerful mineralization inhibitor - could decrease meniscus calcification and ameliorate injury-induced OA lesions (Cheung et al. 2006). Furthermore, it is known that acute trauma and inflammation lead to HO (Kraft et al. 2016). We observed that the loss of SP and $\alpha$ CGRP aggravated the ossification of the meniscal tissue. Owing to their normal triggering effects on osteoblasts and their precursors, a decrease in ectopic bone formation may be predicted in mice with genetic neuropeptide deletion. It was observed in a $\mathrm{HO}$ model that SP facilitated heterotopic ossification in the Achilles tendon while $\alpha$ CGRP alone did not induce such a reactionary adaptation (Tuzmen et al. 2018). They confirmed that a combination of SP and $\alpha$ CGRP demonstrated an inhibitory effect of $\alpha$ CGRP on SP-induced heterotopic ossification (Tuzmen et al. 2018). The observed effect that is attributed to the intense crosstalk of both neuropeptides, and most likely occurs in our wildtype mice, can be limited in case only one sensory neuropeptide is present. Indeed, the interaction between SP and $\alpha \mathrm{CGRP}$ on BMP2-induced bone differentiation has verified that $\alpha$ CGRP could mitigate augmenting effects of SP on BMP-2 signaling, indicating a potential crosstalk between the two neuropeptides (Tuzmen and Campbell 2018). Hence, the increased heterotopic ossification of the meniscus in knockout mice could be the result of an intense disparity between the neuropeptide expression, as measured by the serum concentrations of both neuropeptide-deficient mice (Muschter et al. 2020).

In WT mice, a trend of SB sclerosis was detected two and 8 weeks after the DMM surgery. Other studies have confirmed that 5 weeks after the surgery, bone sclerosis occurred in the medial condyle of the DMM mice, and concluded that SB changes might occur at the same time or possibly earlier than AC changes (Fang et al. 2018). It is suggested that in late-stage OA, the prominent mechanism for SB sclerosis is the elevated bone formation along the cortical end-plate (Jia et al. 2018). As for the effect of neuropeptides, it was found that the OA induction in SP-deficient mice led to an early trend of increased SB sclerosis compared to the Tac1-/- Sham mice. In the late post-traumatic stage, not only this trend became significant, but also a $55 \%$ increased bone formation was detected in the medial epiphysis of the Tac1-/- DMM mice compared to the WT DMM group, suggesting that the DMM surgery aggravated the SB sclerosis that was driven by SPdeficiency. The influence of $\alpha$ CGRP on bone homeostasis was even more prevalent, as indicated by a distinct bone phenotype in aCGRP-deficient mice. Independent from OA induction, $\alpha \mathrm{CGRP}$-/-Sham mice revealed an explicit age-related SB sclerotic phenotype 
that was driven by a thickening of the SB trabeculae to such an extent that some adjacent trabeculae became confluent. The DMM surgery elevated this sclerotic phenotype after eight weeks to the levels comparable to SP-deficient mice.

The medial meniscus destabilization contributed to the formation of osteophytes as early as 2 weeks after the surgery. At the late post-traumatic stage, osteophytes grew in size, appeared as abnormal patellar bone surfaces at the outermost edge of the joint, and were integrated with the medial condyle, leading to the lengthening of the medial tibial plateau. Other studies of injury murine models have also reported the formation of osteophytes at the site of the surgery, while interpreting it as a compensatory reaction to the mechanical debilitation of the damaged cartilage (Huang et al. 2017; Fang et al. 2018; McCulloch et al. 2019). Indeed, it observed that osteophyte formation led to compensatory reactions in the joint morphology by increasing the length of the tibial plateaus, which was interestingly more complex in the neuropeptide-deficient mice. In the Tac1-/- mice, the length of the tibial plateaus was increased both medially and laterally, while the WT mice only exhibited medial tibial plateau adjustments. This is in accordance with other reports regarding the crucial functionality of substance $\mathrm{P}$ in sustaining the physiology and biomechanical characteristics of bone, in particular after acute injuries (Niedermair et al. 2014). Similarly, it has been shown that the basic immunoreactivity of the substance $P$ in SB is low but is significantly enhanced shortly after arthritis induction, and where bone erosion and osteophyte formation occurs (Schwab et al. 1997; Suri et al. 2007; Ogino et al. 2009). As for the influence of the $\alpha$ CGRP, general alterations in bone morphology were observed, as indicated by a smaller lateral tibia plateau compared to the WT Sham mice. OA induction resulted in a compensatory increase of the lateral tibial length in aCGRP -/- mice, which was still lower when compared to its WT and (in particular) Tac1-/- counterparts. Interestingly, measurement of the CC layer revealed that eight weeks after the DMM surgery, $\alpha$ CGRP -/- had the highest CC thickness in the lateral condyle compared to the WT and (in particular) Tac1-/- DMM groups. Hence, it can be concluded that the trauma-induced compensatory reactions in the lateral condyle were manifested as increased CC layer and increased tibial condyle length in the $\alpha \mathrm{CGRP}-/-$ and Tac $1-/-$ mice, respectively.

Aside from the aforementioned SB structural changes, possible cartilage phenotypes were evaluated by measuring the AC stiffness in different zones (i.e. superficial zone, middle zone, and basal zone) with the aid of an indentation-type atomic force microscopy (performed at the Laboratory of Experimental Surgery and Regenerative Medicine, 
Ludwig-Maximilians-University, Munich, Germany). It was observed that the superficial zone of the SP-deficient mice had a surprisingly high matrix stiffness that was reduced in the post-traumatic phase, altering the proteoglycan and the collagen segment of the AC (Muschter et al. 2020). This adaptation in AC rigidity may clarify why SP-deficient mice demonstrated a delayed progression of cartilage degradation, as indicated by the similarity of the OARSI scores of the wildtype and Tac1-/- DMM mice in later stages of the OA (Muschter et al. 2020). In this line of thought, it has been shown that lumbar facetjoint OA that was induced experimentally led to an elevation of SP immunoreactivity in the superficial region of the AC in ipsilateral knee (Duarte et al. 2020). While SP is known to play a prominent role in the mechanical response of cartilage, fracture healing, and pathological cartilage degeneration, its upregulation in AC most probably promotes a pro-catabolic cartilage degradation by stimulating the expression of MMP-13 (Duarte et al. 2020; Li et al. 2020). substance P can also serve anti-anabolic roles akin to those inhibiting the stimulation and deposition of proteoglycan caused by BMP7 in adult human articular chondrocytes (Im et al. 2008). Overall, these studies indicate a protective effect of a decreased substance $\mathrm{P}$ expression on cartilage preservation after traumatic injury, which is consistent with the findings of this study (Tac1-/- mice exhibited a delayed OA onset). It can be further asserted that the function of SP is a highly context- and tissuedependent.

Compared to the to Tac1-/-mice, the stiffness of the AC's superficial zone in aCGRP-/mice displayed an age-related increase, which elevated further after the OA induction (Muschter et al. 2020). This characteristic was similar to the WT mice, suggesting that the influence of $\alpha \mathrm{CGRP}$ is predominantly on bone (micro)structure and to a lesser extent on cartilage homeostasis. Indeed, $\alpha$ CGRP is reported to be an important trophic mediator with anabolic functions in bone physiology and regeneration (Devesa et al. 2014; Appelt et al. 2020). Frequent and dense SB innervation by $\alpha$ CGRP-positive nerve fibers has been documented, but rarely by SP-positive nerves (Schwab et al. 1997). Compared to the SP, the effects of $\alpha$ CGRP on bone cells are more straightforward, inhibiting the osteoclast number after trauma and positively adjusting the osteoblast differentiation and functionality (Grässel and Muschter 2017). In general, the data evidently indicate that changes in the availability of neuropeptides (in particular SP) could result in structural changes in SB and cartilage that could further lead to the progression of OA. Interestingly, it has been shown that loss of joint innervation always preceded cartilage degeneration in C57BL/6 (NIA) mice, and that surgical ablation of joint innervation led to spontaneous 
patellofemoral end-stage OA (Salo et al. 2002a). A similar observation has been made fora rat OA model (Salo et al. 2002b). This raises the question of whether SP and $\alpha$ CGRP are eligible biomarkers to be translated in OA clinical research. In a pre-clinical OA model, a neutralizing antibody to $\alpha$ CGRP demonstrated promising results for ameliorating OA pains, which, however, was not followed up to assess long-term structural changes (Benschop et al. 2014). Likewise, SP and aCGRP serum concentrations did not appear to be associated with OA progression, even though substance $\mathrm{P}$ concentrations were decreased in early $\mathrm{OA}$ by trend, making the eligibility of both neuropeptides as biomarkers ambivalent (Muschter et al. 2020).

In conclusion, it is demonstrated that in the late post-traumatic stage, the medial CC thickness is increased irrespective of the genotype. As calcified cartilage has lower elastic characteristics compared to the $\mathrm{AC}$, this change may have an effect on the $\mathrm{AC}$ response to mechanical impacts, as well as the interlocking of the cartilage with the underlying SB. Aside from an increased cartilage calcification, OA induction leads to heterotopic ossification of the meniscus as well, which is exacerbated by the absence of both SP and $\alpha \mathrm{CGRP}$. Therefore, the calcification process is a common theme in late-stage OA, affecting the biomechanics of the whole joint, and may play an active role in OA pathogenesis. Signs of bone sclerosis is observed as early as 2 weeks post trauma at the site of the injury. The DMM-induced OA has an aggravating effect on subchondral bone sclerosis in SP-deficient mice. Additionally, compared to the aCGRP-deficient mice, joint morphology alterations described by enlargement of the lateral tibial plateau as a counteractive response to DMM surgery is more conspicuous in Tac1-/- mice, which highlights the post-traumatic role of substance $\mathrm{P}$ in bone adaptation. Deficiency of $\alpha$ CGRP contributes to general changes in bone morphology (e.g. smaller diameter of the medial tibia) and SB sclerosis associated with age. At the late-stage post trauma, the formation of osteophytes is not influenced by the neuropeptides. The findings of this study suggest that the SB undergoes major structural changes in the early and late posttraumatic stages, and that both sensory neuropeptides, SP and $\alpha$ CGRP, are important in physiological tissue maintenance, with SP being an essential modulator of AC homeostasis and bone under acute trauma, while aCGRP having a predominant preservative role in bone development under pathophysiological conditions. 


\section{$5 \quad$ Limitations}

\subsection{Maturation-based changes of the subchondral bone}

The limitations of this study include the small sample size for each set of experiment (n =3), which limits the statistical power of the analyses. Additionally, the assessment of the SB microchannels was only carried out qualitatively, owing to a lack of established quantitative protocol during the running time of the project.

\subsection{Mapping of the SB microchannels in healthy and early $\mathrm{OA}$ human femurs}

These studies contain some limitations. First, the measurement of the SB microchannels was performed in sequential 2D slices rather than directly in 3D-reconstriced models. This was due to the extreme sizes of the reconstructed samples, which were typically in the range of 20-30 gigabytes each, thus required a tremendous processing capacity. Consequently, the size and the morphological index might be influenced by the relative angle of the microchannels with the tidemark. Since diagonal microchannels are surveyed by a transverse plane of sectioning, the possibility of an overestimation cannot be overlooked. Nonetheless, since the microchannels reach to the uppermost SB surface, their relative orientation to the tidemark is perpendicular at the AC-SB interface, which minimizes this possible effect. Second, the samples used in these studies were received from the MHH's anatomical gift programme as formalin-fixed whole femurs wrapped in sealed plastic bags. It is reported that formalin fixation and dehydration can result in an nonlinear cartilage tissue shrinkage (Pham and Hull 2007; Kansu et al. 2017). Thus, even though each cartilage-bone biopsy was rehydrated after extraction, the measured AC thicknesses were most likely different from the true values at the physiological conditions. Nonetheless, as the samples were stored in identical conditions, it is postulated that the between-subject effects are minimal, making it valid to study AC thickness differences between the healthy and the early OA groups. Third, the age and sex varied between our participants in the gait analysis (young men) and the cadaveric femurs that were used for micro-CT imaging (older females). During walking, these two populations can potentially exhibit different femoral head loadings. Previous investigations on level ground walking 
have indicated that women show on average (ca. 2-4 degrees) a higher pelvic list, adduction and external hip rotation compared to men during early stance, as well as a higher internal hip rotation between mid to late stance (Smith et al. 2002; Chumanov et al. 2008; Phinyomark et al. 2016; Ro et al. 2017). Similarly, loading on the joint can be influenced by age, as reported by an increased hip flexion during early stance (ca. 2-5 degrees), reduced hip extension between mid to late stance (ca. 5-7 degrees), and decreased hip adduction during early stance (ca. 3 degrees) in older population (Kerrigan et al. 2001; Anderson and Madigan 2014; Qiao et al. 2018). Such variations in the pelvis and femur relative rotations can affect the load mapping of the femoral head during walking. However, these sex- and age-related differences are unlikely to influence our findings, given their limited magnitudes as well as our description of the LBR, which conservatively covers the estimated region of loading (Figure 17). Additionally, in aforementioned studies on the ageing effect, the comparison was made between individuals in their eighth to ninth decades of life and those in their third decade of life (Kerrigan et al. 2001; Anderson and Madigan 2014; Qiao et al. 2018). As our cadaveric samples were approximately aged between 40-60 years, we postulate that the effects associated with age will be lower than the reported values in the literature.

\subsection{Early and late subchondral bone changes in a traumatic OA model}

The limitations of this study include a lack of using littermate mice, which may potentially contribute to misinterpretation or overinterpretation regarding how and to what extent the described effects are due to neuropeptide-deficiency. It is reported that up to ten generations, the gene of interest as well as the linked chromosomal fragments can still be detected after knockout backcrossing is transferred (Holmdahl and Malissen 2012). For comparison with the knockout strains, $\mathrm{C} 57 \mathrm{Bl} / 6 \mathrm{~J}$ mice from were employed in this study (and for backcrossing), which due to epigenetic and environmentally-caused selection may have been different from the backcrossed knockout mice (Holmdahl and Malissen 2012). Nevertheless, the use of littermate mice would have been in conflict with the $3 R$ principles (replacement, reduction, refinement), and impose additional labor burden. In order to minimize the error, animals were matched by age and sex, and were meticulously maintained under the same housing conditions. Moreover, it is reported that background discrepancies mainly influence immunological investigations (Holmdahl and Malissen 2012). Naïve or heterozygous mice were not used due to the same Three Rs guideline 
principles, as well as our interest in purely mechanically-induced alterations, even though heterozygous mice are beneficial for studying deletions that are fatal or induce acute phenotypes at an early developmental phase. On the other hand, deletion of substance P or $\alpha$ CGRP promoted a measurable, yet not overwhelming AC and bone phenotype, while instigating offsetting expression from the corresponding other neuropeptide. Hence, we postulate that the use of heterozygous mice would have been of limited use, rather complicating the data interpretation. 


\section{Conclusion}

Subchondral bone is known to play an important role in the homeostasis and integrity of the joint owing to its intimate interlocking with the articular cartilage. In this dissertation, it is demonstrated that the intricacy of the AC-SB interface is even more complex than originally thought (Figure 37). By developing methods based on high-resolution imaging, not only evasive anatomical features such as cartilage-bone marrow microchannel connectors (CMMC) were three-dimensionally detected and quantitatively described for the first time, but also other important (micro)structural changes of the SB were observed in maturation, idiopathic early osteoarthritic, and early and late traumatic OA. In maturation, the microarchitecture, mineralization, and material composition of the SB undergo critical changes even in $50-\mu \mathrm{m}$ intervals. Specifically, calcified cartilage has extremely dynamic composition characteristics. Maturation of SB is mainly manifested by an increase of the bone volume fraction and SB thickness. In traumatic OA, the calcified cartilage is thickened at the site of the injury during late stages, while traumainduced calcification occurs in the meniscus, and appears as an even stronger phenotype in neuropeptide-deficient mice. Hence, the calcification process is a recurring theme in late-stage $\mathrm{OA}$, affecting the biomechanics of the whole joint, and may play an active role in OA pathogenesis. SB sclerosis, as well as compensatory reactions in the joint such as the change of the tibial and lateral condyle lengths occur in early and late post-traumatic phases. It could be demonstrated that both Substance P and $\alpha$ CGRP are essential for skeletal homeostasis. In adult physiological human femoral heads, CMMC is a prominent load-dependent feature of the SB microarchitecture, and while their capacity for bloodborne nutrition seems to be finite, their colocalization with areas of high local stress, high blood flow rate, and high cartilage thickness, provides support for the hypothesis that the CMMC are passages for interstitial fluid movement between the cartilage and the medullary cavity in order to supply deeper chondrocytes with nutrition. The first reports of quantitative CMMC metrics in healthy and early idiopathic OA human joints can be very helpful for future studies regarding cartilage nutrition and interpretation of former studies. The fact that CMMC go through early microstructural changes in OA makes them a particularly interesting focal point for future investigations on OA etiology, as well as developing novel therapeutic strategies. Therefore, It is proposed that these oftenoverlooked microstructures should be considered in the revised model of the 
osteochondral junction (Figure 37) in order to achieve better diagnostic and treatment strategies for pathological joint disorders.

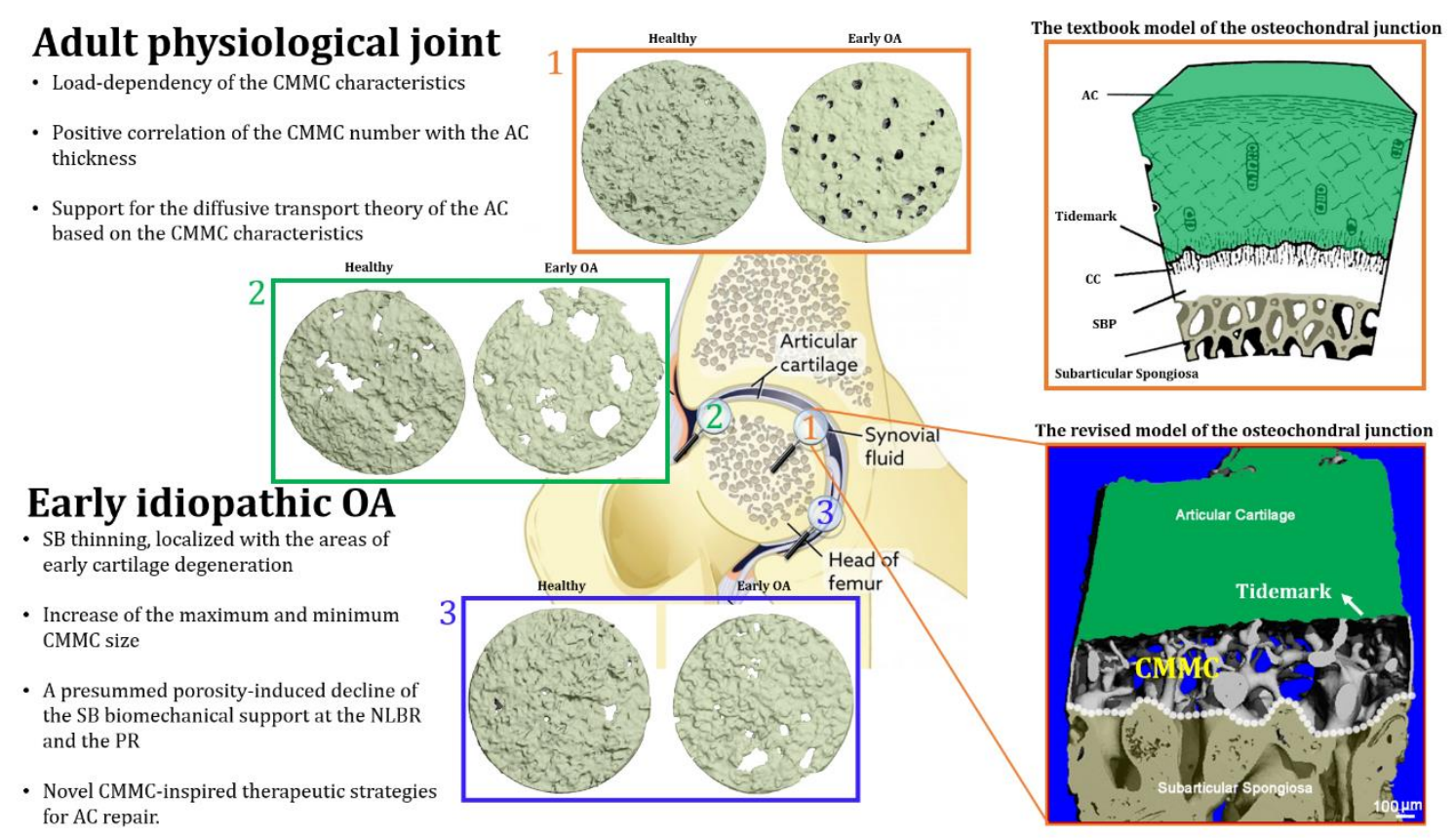

\section{Early and late traumatic $\mathrm{OA}$}
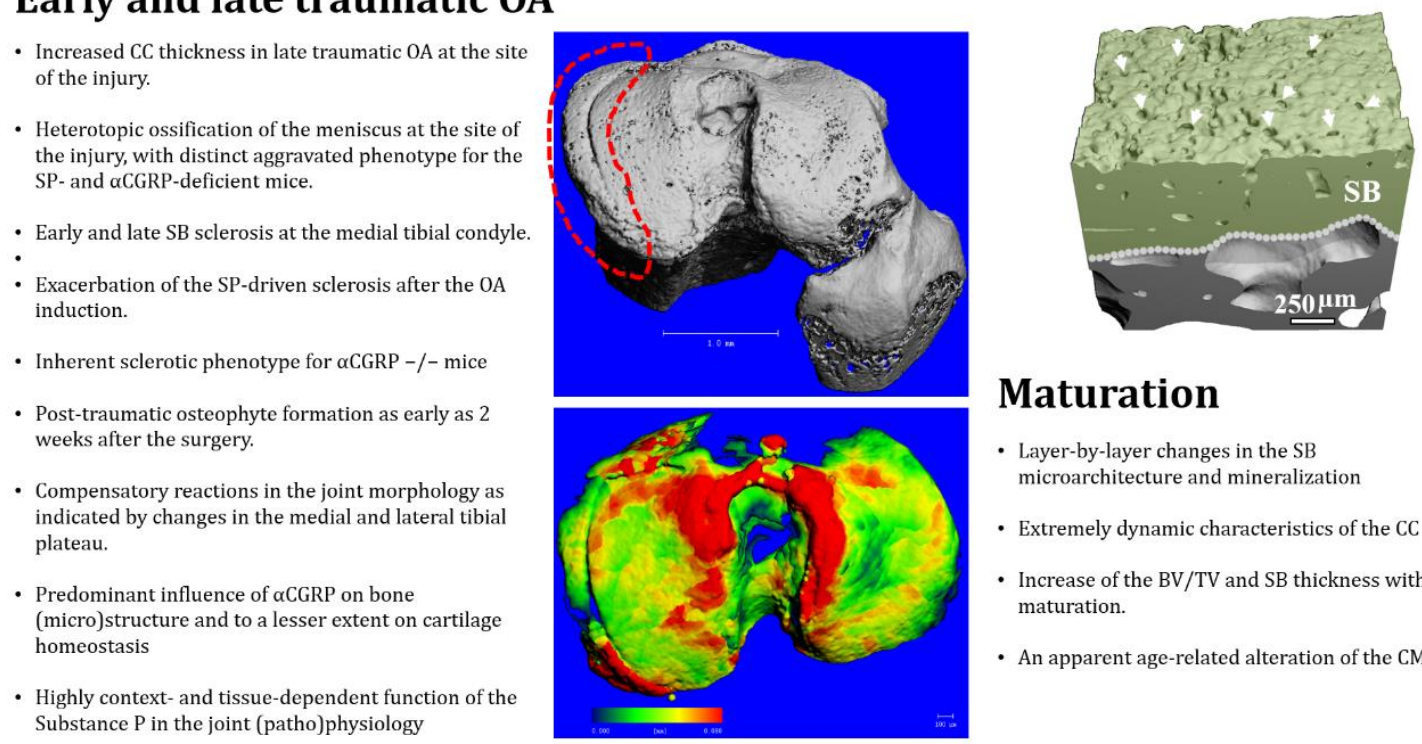

\section{Maturation}

- Layer-by-layer changes in the SB microarchitecture and mineralization

- Extremely dynamic characteristics of the CC

- Increase of the BV/TV and SB thickness with maturation.

- An apparent age-related alteration of the CMMC

Figure 37. The revised model of the osteochondral unit. The CMMC have distinct microarchitectural characteristics based on the location on the joint; i.e. the LBR (1) is characterized by a small-sized, abundant microchannel network. The CMMC number is reduced at the PR (2) and NLBR (3) of the joint, whereas their size is increased. In early OA, increased porosity that is induced by CMMC enlargement is observed. Hence, the CMMC are now included in the revised model of the osteochondral unit. Key conclusion points for the maturation phase, as well as the early and late traumatic OA are presented as bullet points, while the role of sensory neuropeptides Substance P and $\alpha$ CGRP in skeletal homeostasis is highlighted. 


\section{Summary}

Osteochondral unit is emerging as a focal point in osteoarthritis (OA) research, with subchondral bone (SB) attracting more attention as a critical contributing factor to the initiation and progression of OA. In particular, evaluation of the SB microarchitecture has become vital for a deeper understanding of its intimate interrelationship with articular cartilage (AC), for unravelling OA pathogenesis, and for developing future clinical interventions. The aim of this thesis was to investigate the role of the SB (micro)architecture in joint physiology and pathophysiology by developing methods based on high-resolution imaging to quantitatively describe the SB microarchitecture in maturation, normal physiological human joint, early idiopathic OA, and early and late traumatic OA. Specifically, I established a protocol to systematically define, extract, and scan small cartilage-bone cylinders at a resolution of $1.2 \mu \mathrm{m}$, which allowed me to detect a complex microchannel network that connected the medullary cavity to the basal cartilage (CMMC), and to create and quantify an unprecedentedly detailed 3D map of the SB microarchitecture in healthy and early OA human femoral heads. Furthermore, a segmentation method based on a modified Seeded Region Growing technique was developed to semi-automatically calculate the thickness of the calcified cartilage layer in the traumatic OA model.

A layer-by-layer analysis of the SB during maturation revealed intricate developmental changes of the microarchitecture and mineralization. In particular, it was found that calcified cartilage should be considered as a prime focus of investigation owing to its steep mineralization profile and dynamic composition characteristics. The CMMC demonstrated maturation-dependent morphological changes, being small and abundant in young ages while less frequent and enlarged in early adulthood. By profiling the CMMC in normal physiological human femoral heads, a strong association with loading areas was found. In general, the CMMC were small, circular, and with high local density in the load-bearing region, while intermittent, irregularly-shaped, and significantly enlarged in the non-load-bearing region and the peripheral rim of the joint. The unresolved contradictions in the literature such as whether CMMC advance through the calcified cartilage layer, and if they are inherently a pathological feature or not were answered and discussed thoroughly. Likewise, I showed why traditional histology / 
immunocytochemistry methods based on coronal sectioning of the cartilage-bone unit are lacking for examining the complex, spatial distribution of the CMMC.

SB thinning was observed in early idiopathic OA as a localized occurrence linked with areas of partial chondral defect. Moreover, an increased porosity of the SB was detected compared to the healthy group, which was mainly induced by enlargement of the CMMC. Based on these observations, a new hypothesis regarding the role of SB microarchitecture was introduced that may also explain why early AC degradation does not predominantly occur at the load-bearing areas. Additionally, I discuss the idea of a novel therapeutic invention based on the concept of CMMC that can potentially address today's insufficiencies of common treatment methods such as microfracturing. I could also show several (micro)structural changes of the SB in a traumatic murine OA model. In particular, while investigating the role of sensory neuropeptides Substance P and $\alpha$ CGRP in tissue maintenance. A lack of $\alpha$ CGRP led to a distinct bone phenotype with a marked SB sclerosis. DMM surgery aggravated SB sclerosis in both Substance P -/- and aCGRP -/- models, while osteophyte formation and an calcified cartilage thickness increase were detected as compensatory reactions to trauma. Likewise, increased heterotopic ossification of the meniscus was observed due to the strong imbalanced expression of the neuropeptides. It is therefore concluded that the AC-SB interface is even more intricate than originally thought, while a revised model for the osteochondral junction is proposed. 


\section{Bibliography}

Adams R, Bischof L (1994): Seeded Region Growing. IEEE Trans Pattern Anal Mach Intell 16, $641-647$

Alini M, Matsui Y, Dodge GR, Poole AR (1992): The extracellular matrix of cartilage in the growth plate before and during calcification: Changes in composition and degradation of type II collagen. Calcif Tissue Int $\underline{50}, 327-335$

Anderson DD, Brown TD, Radin EL (1993): The influence of basal cartilage calcification on dynamic juxtaarticular stress transmission. Clin Orthop Relat Res 298-307

Anderson DE, Madigan ML (2014): Healthy older adults have insufficient hip range of motion and plantar flexor strength to walk like healthy young adults. J Biomech $\underline{47,1104-1109}$

Andrea Drusini (1987): Refinements of two methods for the histomorphometric determination of age in human bone. Z Morphol Anthropol 77, 167-176

Appelt J, Baranowsky A, Jahn D, Yorgan T, Köhli P, Otto E, Farahani SK, Graef F, Fuchs M, Herrera A, et al. (2020): The neuropeptide calcitonin gene-related peptide alpha is essential for bone healing. EBioMedicine $\underline{59}$

Arkill KP, Winlove CP (2008): Solute transport in the deep and calcified zones of articular cartilage. Osteoarthr Cartil 16, 708-714

Ateshian GA (2009): The Role of Interstitial Fluid Pressurization in Articular Cartilage Lubrication. J Biomech $\underline{42}, 1163$

Band MR, Larson JH, Rebeiz M, Green CA, Heyen DW, Donovan J, Windish R, Steining C, Mahyuddin P, Womack JE, Lewin HA (2000): An ordered comparative map of the cattle and human genomes. Genome Res $\underline{10}, 1359-68$

Batiste DL, Kirkley A, Laverty S, Thain LMF, Spouge AR, Holdsworth DW (2004): Ex vivo characterization of articular cartilage and bone lesions in a rabbit ACL transection model of osteoarthritis using MRI and micro-CT. Osteoarthr Cartil 12, 986-996

Bellido M, Lugo L, Roman-Blas JA, Castañeda S, Caeiro JR, Dapia S, Calvo E, Largo R, Herrero-Beaumont G (2010): Subchondral bone microstructural damage by increased remodelling aggravates experimental osteoarthritis preceded by osteoporosis. Arthritis Res Ther $\underline{12}$ 
Benschop RJ, Collins EC, Darling RJ, Allan BW, Leung D, Conner EM, Nelson J, Gaynor B, Xu J, Wang XF, et al. (2014): Development of a novel antibody to calcitonin gene-related peptide for the treatment of osteoarthritis-related pain. Osteoarthr Cartil 22, 578-585

Bergmann G, Deuretzbacher G, Heller M, Graichen F, Rohlmann A, Strauss J, Duda G. (2001): Hip contact forces and gait patterns from routine activities. J Biomech $\underline{34}, 859-871$

Berry JL, Thaeler-Oberdoerster DA, Greenwald AS (1986): Subchondral pathways to the superior surface of the human talus. Foot Ankle $\underline{7}, 2-9$

Bettica P, Cline G, Hart DJ, Meyer J, Spector TD (2002): Evidence for increased bone resorption in patients with progressive knee osteoarthritis: Longitudinal results from the Chingford study. Arthritis Rheum $\underline{46}, 3178-3184$

Bian Q, Jain A, Xu X, Kebaish K, Crane JL, Zhang Z, Wan M, Ma L, Riley LH, Sponseller PD, et al. (2016): Excessive Activation of TGF $\beta$ by Spinal Instability Causes Vertebral Endplate Sclerosis. Sci Rep $\underline{6}, 1-10$

Björkström S, Goldie IF (1980): A study of the arterial supply of the patella in the normal state, in chondromalacia patellae and in osteoarthrosis. Acta Orthop Scand 51, 63-70

Bobinac D, Spanjol J, Zoricic S, Maric I (2003): Changes in articular cartilage and subchondral bone histomorphometry in osteoarthritic knee joints in humans. Bone $\underline{32}, 284-90$

Bonjour JP (2011): Calcium and phosphate: A duet of ions playing for bone health. J Am Coll Nutr $\underline{30}, 438 \mathrm{~S}-448 \mathrm{~S}$

Bonnet CS, Walsh DA (2005): Osteoarthritis, angiogenesis and inflammation. Rheumatology $\underline{44}, 7-16$

Botter SM, Van Osch GJVM, Clockaerts S, Waarsing JH, Weinans H, Van Leeuwen JPTM (2011): Osteoarthritis induction leads to early and temporal subchondral plate porosity in the tibial plateau of mice: An in vivo microfocal computed tomography study. Arthritis Rheum $\underline{63}, 2690-2699$

Boudenot A, Presle N, Uzbekov R, Toumi H, Pallu S, Lespessailles E (2014): Effect of intervaltraining exercise on subchondral bone in a chemically-induced osteoarthritis model. Osteoarthr Cartil 22, 1176-1185

Brodin H (1954): Paths of nutrition in articular cartilage and intervertebral discs. Acta Orthop $\underline{24}, 177-183$

Brown TD, Vrahas MS (1984): The apparent elastic modulus of the juxtaricular subchondral bone of the femoral head. J Orthop Res 2, 32-38 
Buckland-Wright C (2004): Subchondral bone changes in hand and knee osteoarthritis detected by radiography. Osteoarthr Cartil $\underline{12}, 10-19$

Burgstaller JP, Brem G (2017): Aging of Cloned Animals: A Mini-Review. Gerontology $\underline{63}$, $417-425$

Burr DB, Radin EL (2003): Microfractures and microcracks in subchondral bone: Are they relevant to osteoarthrosis? Rheum Dis Clin North Am $\underline{29}$, 675-685

Burr DB, Gallant MA (2012): Bone remodelling in osteoarthritis. Nat Rev Rheumatol $\underline{8}$, 665673

Byers PD, Contepomi CA, Farkas TA (1970): A post mortem study of the hip joint. Including the prevalence of the features of the right side. Ann Rheum Dis $\underline{29}, 15-31$

Campos PF, Gilbert TMP: DNA Extraction from Formalin-Fixed Material. In: Methods in molecular biology (Clifton, N.J.). Band 840; 2012, 81-85

Carnelli D, Vena P, Dao M, Ortiz C, Contro R (2013): Orientation and size-dependent mechanical modulation within individual secondary osteons in cortical bone tissue. J R Soc Interface $\underline{10}$

Castañeda S, Roman-Blas JA, Largo R, Herrero-Beaumont G (2012): Subchondral bone as a key target for osteoarthritis treatment. Biochem Pharmacol $\underline{83}, 315-323$

Chen D, Shen J, Zhao W, Wang T, Han L, Hamilton JL, Im H-J (2017): Osteoarthritis: toward a comprehensive understanding of pathological mechanism. Bone Res $\underline{5}, 16044$

Chen H, Zhou X, Shoumura S, Emura S, Bunai Y (2010): Age- and gender-dependent changes in three-dimensional microstructure of cortical and trabecular bone at the human femoral neck. Osteoporos Int $\underline{21}, 627-636$

Chen H, Zhou X, Fujita H, Onozuka M, Kubo KY (2013): Age-related changes in trabecular and cortical bone microstructure. Int J Endocrinol 2013, 1-9

Cheung HS, Sallis JD, Demadis KD, Wierzbicki A (2006): Phosphocitrate blocks calcificationinduced articular joint degeneration in a guinea pig model. Arthritis Rheum 54, 2452-2461

Chevrier A, Kouao ASM, Picard G, Hurtig MB, Buschmann MD (2015): Interspecies comparison of subchondral bone properties important for cartilage repair. J Orthop Res $\underline{33}$, $63-70$

Chiba K, Ito M, Osaki M, Uetani M, Shindo H (2011): In vivo structural analysis of subchondral trabecular bone in osteoarthritis of the hip using multi-detector row CT. Osteoarthr Cartil $\underline{19}, 180-185$ 
Chiu H-C, Chang H-Y, Mau L-W, Lee T-K, Liu H-W (2000): Height, Weight, and Body Mass Index of Elderly Persons in Taiwan. Journals Gerontol Ser A Biol Sci Med Sci 55, M684M690

Choi WJ, Park KK, Kim BS, Lee JW (2009): Osteochondral lesion of the talus: Is There a critical defect size for poor outcome? Am J Sports Med 37, 1974-1980

Christensen P, Kjær J, Sneppen O, Vang PS, Melsen F, Nielsen HE (1982): the subchondral bone of the proximal tibial epiphysis in osteoarthritis of the knee. Acta Orthop $\underline{53}, 889-895$

Chu L, He Z, Qu X, Liu X, Zhang W, Zhang Shuo, Han X, Yan M, Xu Q, Zhang Shuhong, et al. (2020): Different subchondral trabecular bone microstructure and biomechanical properties between developmental dysplasia of the hip and primary osteoarthritis. J Orthop Transl 22, 50-57

Chumanov ES, Wall-Scheffler C, Heiderscheit BC (2008): Gender differences in walking and running on level and inclined surfaces. Clin Biomech $\underline{23}, 1260-1268$

Clark JM (1990): The structure of vascular channels in the subchondral plate. J Anat $\underline{171}, 105-$ 115

Clark JM, Huber JD (1990): The structure of the human subchondral plate. J Bone Joint Surg Br $\underline{72}, 866-73$

Collins D (1949): The Pathology of Articular and Spinal Diseases. Edward Arnold Co

Costagliola A, Wojcik S, Pagano TB, De Biase D, Russo V, Iovane V, Grieco E, Papparella S, Paciello O (2016): Age-Related Changes in Skeletal Muscle of Cattle. Vet Pathol 53, 436446

Cowin SC, Cardoso L (2015): Blood and interstitial flow in the hierarchical pore space architecture of bone tissue. J Biomech $\underline{48}, 842-54$

Crisafuli FAP, Ramos EB, Rocha MS (2015): Characterizing the interaction between DNA and GelRed fluorescent stain. Eur Biophys J $\underline{44}, 1-7$

Curtis TA, Ashrafi SH, Weber DF (1985): Canalicular communication in the cortices of human long bones. Anat Rec 212, 336-344

Day JS, Ding M, van der Linden JC, Hvid I, Sumner DR, Weinans H (2001): A decreased subchondral trabecular bone tissue elastic modulus is associated with pre-arthritic cartilage damage. J Orthop Res 19, 914-918

de Windt TS, Vonk LA, Brittberg M, Saris DBF (2013): Treatment and Prevention of (Early) Osteoarthritis Using Articular Cartilage Repair-Fact or Fiction? A Systematic Review. Cartilage $\underline{4}, 5 \mathrm{~S}$ 
Delp SL, Anderson FC, Arnold AS, Loan P, Habib A, John CT, Guendelman E, Thelen DG (2007): OpenSim: Open-Source Software to Create and Analyze Dynamic Simulations of Movement. IEEE Trans Biomed Eng 54, 1940-1950

Deng B, Wang F, Yin L, Chen C, Guo L, Chen H, Gong X, Li Y, Yang L (2016): Quantitative study on morphology of calcified cartilage zone in OARSI $0 \sim 4$ cartilage from osteoarthritic knees. Curr Res Transl Med 64, 149-154

Devesa I, Ferrándiz-Huertas C, Mathivanan S, Wolf C, Luján R, Changeux JP, Ferrer-Montiel A (2014): $\alpha$ CGRP is essential for algesic exocytotic mobilization of TRPV1 channels in peptidergic nociceptors. Proc Natl Acad Sci U S A $\underline{111}$, 18345-18350

Diehr P, O’Meara ES, Fitzpatrick A, Newman AB, Kuller L, Burke G (2008): Weight, mortality, years of healthy life, and active life expectancy in older adults. J Am Geriatr Soc $\underline{56}, 76-83$

Ding M (2010): Microarchitectural adaptations in aging and osteoarthrotic subchondral bone issues. Acta Orthop $\underline{81}, 1-53$

Ding M, Odgaard A, Hvid I (2003): Changes in the three-dimensional microstructure of human tibial cancellous bone in early osteoarthritis. J Bone Jt Surg - Ser B $\underline{85}, 906-12$

Dingemanse W, Müller-Gerbl M, Jonkers I, Sloten J Vander, van Bree H, Gielen I (2017): A prospective follow up of age related changes in the subchondral bone density of the talus of healthy Labrador Retrievers. BMC Vet Res 13, 57

Donell S (2019): Subchondral bone remodelling in osteoarthritis. EFORT Open Rev 4, 221-229

Dong X, Milholland B, Vijg J (2016): Evidence for a limit to human lifespan. Nature 538, 257259

Duarte FCK, Zwambag DP, Brown SHM, Clark A, Hurtig M, Srbely JZ (2020): Increased Substance P Immunoreactivity in Ipsilateral Knee Cartilage of Rats Exposed to Lumbar Spine Injury. Cartilage 11, 251-261

Duncan H, Jundt J, Riddle JM, Pitchford W, Christopherson T (1987): The tibial subchondral plate. A scanning electron microscopic study. J Bone Joint Surg Am $\underline{69}, 1212-20$

Dunlop DD, Manheim LM, Song J, Chang RW (2001): Arthritis prevalence and activity limitations in older adults. Arthritis Rheum 44, 212-221

Ea H-K, Nguyen C, Bazin D, Bianchi A, Guicheux J, Reboul P, Daudon M, Lioté F (2011): Articular cartilage calcification in osteoarthritis: Insights into crystal-induced stress. Arthritis Rheum $\underline{63}, 10-18$ 
Eckstein F, Müller-Gerbl M, Putz R (1992): Distribution of subchondral bone density and cartilage thickness in the human patella. J Anat $\underline{180 \text { ( Pt 3 }, 425-33}$

Eitner A, Hofmann GO, Schaible HG (2017): Mechanisms of osteoarthritic pain. Studies in humans and experimental models. Front Mol Neurosci 10, 349

Emes RD, Goodstadt L, Winter EE, Ponting CP (2003): Comparison of the genomes of human and mouse lays the foundation of genome zoology. Hum Mol Genet 12, 701-709

Erben RG (1997): Embedding of bone samples in methylmethacrylate: An improved method suitable for bone histomorphometry, histochemistry, and immunohistochemistry. J Histochem Cytochem 45, 307-313

Evans ACO, Davies FJ, Nasser LF, Bowman P, Rawlings NC (1995): Differences in early patterns of gonadotrophin secretion between early and late maturing bulls, and changes in semen characteristics at puberty. Theriogenology $\underline{43}, 569-578$

Fall A, Diop M, Sandford J, Wissocq YJ, Durkin JW, Trail JCM (1982): Evaluation of the productivities of Djallonke sheep and N'Dama cattle at the Centre de Recherches Zootechniques, Kolda, Senegal.

Fam AG, Morava-Protzner I, Purcell C, Young BD, Bunting PS, Lewis AJ (1995): Acceleration of experimental lapine osteoarthritis by calcium pyrophosphate microcrystalline synovitis. Arthritis Rheum 38, 201-210

Fan M, Lee TCM (2015): Variants of seeded region growing. IET Image Process $\underline{9}, 478-485$

Fang H, Huang L, Welch I, Norley C, Holdsworth DW, Beier F, Cai D (2018): Early Changes of Articular Cartilage and Subchondral Bone in The DMM Mouse Model of Osteoarthritis. Sci Rep $\underline{8}, 1-9$

Faul F, Erdfelder E, Lang A-G, Buchner A (2007): G*Power 3: A flexible statistical power analysis program for the social, behavioral, and biomedical sciences. Behav Res Methods $\underline{39}, 175-191$

Fell NLA, Lawless BM, Cox SC, Cooke ME, Eisenstein NM, Shepherd DET, Espino DM (2019): The role of subchondral bone, and its histomorphology, on the dynamic viscoelasticity of cartilage, bone and osteochondral cores. Osteoarthr Cartil 27, 535-543

Felson DT, McLaughlin S, Goggins J, LaValley MP, Gale ME, Totterman S, Li W, Hill C, Gale D (2003): Bone Marrow Edema and Its Relation to Progression of Knee Osteoarthritis. Ann Intern Med $\underline{139}$

Ferrari A, Benedetti MG, Pavan E, Frigo C, Bettinelli D, Rabuffetti M, Crenna P, Leardini A (2008): Quantitative comparison of five current protocols in gait analysis. Gait Posture 28, $207-216$ 
Findlay DM, Kuliwaba JS (2016): Bone-cartilage crosstalk: a conversation for understanding osteoarthritis. Bone Res $\underline{4}, 16028$

Fritton SP, Weinbaum S (2009): Fluid and Solute Transport in Bone: Flow-Induced Mechanotransduction. Annu Rev Fluid Mech 41, 347-374

Funck-Brentano T, Cohen-Solal M (2015): Subchondral bone and osteoarthritis. Curr Opin Rheumatol 27, 420-426

Gianakos AL, Haring RS, Shimozono Y, Fragomen A, Kennedy JG (2020): Effect of Microfracture on Functional Outcomes and Subchondral Sclerosis Following Distraction Arthroplasty of the Ankle Joint. Foot Ankle Int 41, 631-638

Glasson SS, Blanchet TJ, Morris EA (2007): The surgical destabilization of the medial meniscus (DMM) model of osteoarthritis in the 129/SvEv mouse. Osteoarthr Cartil 15, $1061-1069$

Goldring MB, Goldring SR: Articular cartilage and subchondral bone in the pathogenesis of osteoarthritis. In: Annals of the New York Academy of Sciences. Band 1192; Blackwell Publishing Inc. 2010, 230-237

Goldring SR (2009): Role of Bone in Osteoarthritis Pathogenesis. Med Clin North Am 93, 25 35

Grässel S, Muschter D (2017): Peripheral nerve fibers and their neurotransmitters in osteoarthritis pathology. Int J Mol Sci $\underline{18}$

Green WT, Martin GN, Eanes ED, Sokoloff L (1970): Microradiographic study of the calcified layer of articular cartilage. Arch Pathol 90, 151-8

Greenwald AS, Haynes DW (1969): A pathway for nutrients from the medullary cavity to the articular cartilage of the human femoral head. J Bone Joint Surg Br $\underline{51}, 747-53$

Grosskopf B (2004): Leichenbrand - Biologisches und kulturhistorisches Quellenmaterial zur Rekonstruktion vor- und frühgeschichtlicher Populationen und ihrer Funeralpraktiken.

Grynpas MD, Alpert B, Katz I, Lieberman I, Pritzker KPH (1991): Subchondral bone in osteoarthritis. Calcif Tissue Int $\underline{49}, 20-26$

Guccione AA, Felson DT, Anderson JJ, Anthony JM, Zhang Y, Wilson PWF, Kelly-Hayes M, Wolf PA, Kreger BE, Kannel WB (1994): The effects of specific medical conditions on the functional limitations of elders in the Framingham study. Am J Public Health $\underline{84}$, 351-358

Guermazi A, Niu J, Hayashi D, Roemer FW, Englund M, Neogi T, Aliabadi P, McLennan CE, Felson DT (2012): Prevalence of abnormalities in knees detected by MRI in adults without 
knee osteoarthritis: Population based observational study (Framingham Osteoarthritis Study). BMJ $\underline{345}$

Guermazi A, Hayashi D, Roemer FW, Niu J, Quinn EK, Crema MD, Nevitt MC, Torner J, Lewis CE, Felson DT (2017): Partial- and Full-Thickness Focal Cartilage Defects Contribute Equally to Development of New Cartilage Damage in Knee Osteoarthritis: The Multicenter Osteoarthritis Study. Arthritis Rheumatol 69, 560-564

Hagens G Von (1979): Impregnation of soft biological specimens with thermosetting resins and elastomers. Anat Rec 194, 247-255

Ham AW (1952): Some histophysiological problems peculiar to calcified tissues. J Bone Joint Surg Am 24 A, 701-28

Haraldsson S (1959): On Osteochondrosis Deformans Juvenilis Capituli Humeri Including Investigation of Intra-Osseous Vasculature in Distal Humerus. Acta Orthop Scand $\underline{30}, 5$ 232

Hargrave-Thomas E, van Sloun F, Dickinson M, Broom N, Thambyah A (2015): Multi-scalar mechanical testing of the calcified cartilage and subchondral bone comparing healthy vs early degenerative states. Osteoarthr Cartil $\underline{23}, 1755-1762$

Hayami T, Oka M, Ikeuchi K, Nakamura T, Hojo H, Sakaguchi K: The Energy-Absorbing Function of Cancellous Bone and Its Influence on the Loosening of Artificial Joints. In: Clinical Biomechanics and Related Research. Springer Japan 1994, 138-152

Hayashi D, Felson DT, Niu J, Hunter DJ, Roemer FW, Aliabadi P, Guermazi A (2014): Preradiographic osteoarthritic changes are highly prevalent in themedial patella and medial posterior femur in older persons: Framingham OA study. Osteoarthr Cartil 22, 76-83

Henrotin Y, Pesesse L, Sanchez C (2012): Subchondral bone and osteoarthritis: Biological and cellular aspects. Osteoporos Int $\underline{23}$

Hodge JA, McKibbin B (1969): The nutrition of mature and immature cartilage in rabbits. An autoradiographic study. J Bone Joint Surg Br $\underline{51}$, 140-7

Hoemann CD, Lafantaisie-Favreau CH, Lascau-Coman V, Chen G, Guzmán-Morales J (2012): The cartilage-bone interface. J Knee Surg 25, 85-97

Holmdahl DE, Ingelmark BE (1950): The contact between the articular cartilage and the medullary cavities of the bone. Acta Orthop Scand $\underline{20}, 156-65$

Holmdahl R, Malissen B (2012): The need for littermate controls. Eur J Immunol 42, 45-47

Holopainen JT, Brama PAJ, Halmesmäki E, Harjula T, Tuukkanen J, van Weeren PR, Helminen HJ, Hyttinen MM (2008): Changes in subchondral bone mineral density and collagen 
matrix organization in growing horsesSources of funding: Financial support was received from the Ministry of Education in Finland (projects 25/627/2006 and 61/627/2005), Academy of Finland (projects 113112, 213548, and 216231), the National Graduate School of Musculo-Skeletal Diseases in Finland and the North-Savo Fund of the Finnish... Bone 43, $1108-1114$

Hootman JM, Helmick CG (2006): Projections of US prevalence of arthritis and associated activity limitations. Arthritis Rheum 54, 226-229

Huang H, Skelly JD, Ayers DC, Song J (2017): Age-dependent Changes in the Articular Cartilage and Subchondral Bone of C57BL/6 Mice after Surgical Destabilization of Medial Meniscus. Sci Rep 7, 42294

Hunter DJ (2011): Lower extremity osteoarthritis management needs a paradigm shift. Br J Sports Med 45, 283-288

Hunter DJ, Schofield D, Callander E (2014): The individual and socioeconomic impact of osteoarthritis. Nat Rev Rheumatol 10, 437-441

Hwang J, Bae WC, Shieu W, Lewis CW, Bugbee WD, Sah RL (2008): Increased hydraulic conductance of human articular cartilage and subchondral bone plate with progression of osteoarthritis. Arthritis Rheum $\underline{58}, 3831-3842$

Im HJ, Li X, Muddasani P, Kim GH, Davis F, Rangan J, Forsyth CB, Ellman M, Thonar EJMA (2008): Basic fibroblast growth factor accelerates matrix degradation via a neuro-endocrine pathway in human adult articular chondrocytes. J Cell Physiol 215, 452-463

Imhof H, Breitenseher M, Kainberger F, Trattnig S (1997): Degenerative joint disease: cartilage or vascular disease? Skeletal Radiol 26, 398-403

Imhof H, Breitenseher M, Kainberger F, Rand T, Trattnig S (1999): Importance of subchondral bone to articular cartilage in health and disease. Top Magn Reson imaging $\underline{10}, 180-192$

Imhof H, Sulzbacher I, Grampp S, Czerny C, Youssefzadeh S, Kainberger F (2000):

Subchondral bone and cartilage disease: a rediscovered functional unit. Invest Radiol $\underline{35}$, $581-8$

Intema F, Hazewinkel HAW, Gouwens D, Bijlsma JWJ, Weinans H, Lafeber FPJG, Mastbergen SC (2010): In early OA, thinning of the subchondral plate is directly related to cartilage damage: Results from a canine ACLT-meniscectomy model. Osteoarthr Cartil 18, 691-698

Jansen EJ, Emans PJ, Van Rhijn LW, Bulstra SK, Kuijer R (2008): Development of partialthickness articular cartilage injury in a rabbit model. Clin Orthop Relat Res $\underline{466}$, 487-494 
Jia H, Ma X, Wei Y, Tong W, Tower RJ, Chandra A, Wang L, Sun Z, Yang Z, Badar F, et al. (2018): Subchondral bone plate sclerosis during late osteoarthritis is caused by loadinginduced reduction in Sclerostin HHS Public Access. Arthritis Rheumatol 70, 230-241

Kamibayashi L, Wyss UP, Cooke TD, Zee B (1995): Trabecular microstructure in the medial condyle of the proximal tibia of patients with knee osteoarthritis. Bone 17, 27-35

Kansu L, Aydın E, Akkaya H, Avcı S, Akalın N (2017): Shrinkage of nasal mucosa and cartilage during formalin fixation. Balkan Med J $\underline{34}, 458-463$

Kauppinen S, Karhula SS, Thevenot J, Ylitalo T, Rieppo L, Kestilä I, Haapea M, Hadjab I, Finnilä MA, Quenneville E, et al. (2019): 3D morphometric analysis of calcified cartilage properties using micro-computed tomography. Osteoarthr Cartil 27, 172-180

Kawcak CE, McIlwraith CW, Norrdin RW, Park RD, James SP (2001): The role of subchondral bone in joint disease: a review. Equine Vet J $\underline{33}, 120-126$

Kerley ER, Ubelaker DH (1978): Revisions in the microscopic method of estimating age at death in human cortical bone. Am J Phys Anthropol 49, 545-546

Kerrigan DC, Lee LW, Collins JJ, Riley PO, Lipsitz LA (2001): Reduced hip extension during walking: Healthy elderly and fallers versus young adults. Arch Phys Med Rehabil $\underline{82,26-}$ 30

Kraft CT, Agarwal S, Ranganathan K, Wong VW, Loder S, Li J, Delano MJ, Levi B (2016): Trauma-induced heterotopic bone formation and the role of the immune system: A review. J Trauma Acute Care Surg $\underline{\text { 80, }}$ 156-165

Kufahl RH, Saha S (1990): A theoretical model for stress-generated fluid flow in the canaliculilacunae network in bone tissue. J Biomech $\underline{23}, 171-80$

Kuhn LT, Grynpas MD, Rey CC, Wu Y, Ackerman JL, Glimcher MJ (2008): A Comparison of the Physical and Chemical Differences Between Cancellous and Cortical Bovine Bone Mineral at Two Ages. Calcif Tissue Int $\underline{83}, 146-154$

Lajeunesse D, Reboul P (2003): Subchondral bone in osteoarthritis: A biologic link with articular cartilage leading to abnormal remodeling. Curr Opin Rheumatol 15, 628-633

Lane LB, Bullough PG (1980): Age-related changes in the thickness of the calcified zone and the number of tidemarks in adult human articular cartilage. J Bone Joint Surg Br $\underline{62}, 372-5$

Lane LB, Villacin A, Bullough PG (1977): The vascularity and remodelling of subchondrial bone and calcified cartilage in adult human femoral and humeral heads. An age- and stressrelated phenomenon. J Bone Joint Surg Br 59, 272-8 
Lefèvre E, Farlay D, Bala Y, Subtil F, Wolfram U, Rizzo S, Baron C, Zysset P, Pithioux M, Follet H (2019): Compositional and mechanical properties of growing cortical bone tissue: a study of the human fibula. Sci Rep $\underline{9}, 1-16$

Lemirre T, Santschi E, Girard C, Fogarty U, Chapuis L, Richard H, Beauchamp G, Laverty S (2020): Maturation of the equine medial femoral condyle osteochondral unit. Osteoarthr Cartil Open $\underline{2}, 100029$

Li B, Aspden RM (1997): Mechanical and material properties of the subchondral bone plate from the femoral head of patients with osteoarthritis or osteoporosis. Ann Rheum Dis $\underline{56}$, $247-254$

Li FXZ, Xu F, Lin X, Wu F, Zhong JY, Wang Y, Guo B, Zheng MH, Shan SK, Yuan LQ (2020): The Role of Substance $P$ in the Regulation of Bone and Cartilage Metabolic Activity. Front Endocrinol (Lausanne) 11, 77

Li G, Yin J, Gao J, Cheng TS, Pavlos NJ, Zhang C, Zheng MH (2013): Subchondral bone in osteoarthritis: insight into risk factors and microstructural changes. Arthritis Res Ther $\underline{15}$, 223

Lieberman DE, Devlin MJ, Pearson OM (2001): Articular area responses to mechanical loading: Effects of exercise, age, and skeletal location. Am J Phys Anthropol 116, 266-277

Lindsay RM, Alhejaili F, Nesrallah G, Leitch R, Clement L, Heidenheim AP, Kortas C (2003): Calcium and phosphate balance with quotidian hemodialysis. Am J Kidney Dis $\underline{42}$, 24-29

Liu Chenglei, Liu Chang, Si L, Shen H, Wang Q, Yao W (2018): Relationship between subchondral bone microstructure and articular cartilage in the osteoarthritic knee using 3T MRI. J Magn Reson Imaging 48, 669-679

Liu Y, Lian Q, He J, Zhao J, Jin Z, Li D (2011): Study on the Microstructure of Human Articular Cartilage/Bone Interface. J Bionic Eng $\underline{8}$, 251-262

Loeser RF, Goldring SR, Scanzello CR, Goldring MB (2012): Osteoarthritis: a disease of the joint as an organ. Arthritis Rheum $\underline{64}, 1697-707$

Lories RJ, Luyten FP (2011): The bone-cartilage unit in osteoarthritis. Nat Rev Rheumatol 7 , 43-49

Lozupone E, Favia A (1990): The structure of the trabeculae of cancellous bone. 2. Long bones and mastoid. Calcif Tissue Int $\underline{46}, 367-72$

Lu JT, Son YJ, Lee J, Jetton TL, Shiota M, Moscoso L, Niswender KD, Loewy AD, Magnuson MA, Sanes JR, Emeson RB (1999): Mice lacking $\alpha$-calcitonin gene-related peptide exhibit normal cardiovascular regulation and neuromuscular development. Mol Cell Neurosci 14, 99-120 
Luyten FP, Lories RJU, Verschueren P, de Vlam K, Westhovens R (2006): Contemporary concepts of inflammation, damage and repair in rheumatic diseases. Best Pract Res Clin Rheumatol 20, 829-848

Lyons TJ, Stoddart RW, McClure SF, McClure J (2005): The tidemark of the chondro-osseous junction of the normal human knee joint. J Mol Histol $\underline{36}$, 207-215

Lyons TJ, McClure SF, Stoddart RW, McClure J (2006): The normal human chondro-osseous junctional region: Evidence for contact of uncalcified cartilage with subchondral bone and marrow spaces. BMC Musculoskelet Disord ㄱ, 52

Ma HL, Blanchet TJ, Peluso D, Hopkins B, Morris EA, Glasson SS (2007): Osteoarthritis severity is sex dependent in a surgical mouse model. Osteoarthr Cartil $\underline{15}, 695-700$

Madry H, van Dijk CN, Mueller-Gerbl M (2010): The basic science of the subchondral bone. Knee Surgery, Sport Traumatol Arthrosc 18, 419-433

Malinin T, Ouellette EA (2000): Articular cartilage nutrition is mediated by subchondral bone: A long-term autograft study in baboons. Osteoarthr Cartil $\underline{8}, 483-491$

Mankin HJ (1963): The calcified zone (basal layer) of articular cartilage of rabbits. Anat Rec $145,73-87$

Maroudas A (1968): Physicochemical properties of cartilage in the light of ion exchange theory. Biophys J $\underline{8}, 575-95$

Maroudas A, Bullough P, Swanson SA, Freeman MA (1968): The permeability of articular cartilage. J Bone Joint Surg Br 무, 166-77

Martinelli MJ, Eurell J, Les CM, Fyhrie D, Bennett D (2002): Age-related morphometry of equine calcified cartilage. Equine Vet J $\underline{34}, 274-278$

Mastbergen SC, Lafeber FPJG (2011): Changes in subchondral bone early in the development of osteoarthritis. Arthritis Rheum 63, 2561-2563

McCulloch K, Huesa C, Dunning L, Litherland GJ, Van 'T Hof RJ, Lockhart JC, Goodyear CS (2019): Accelerated post traumatic osteoarthritis in a dual injury murine model. Osteoarthr Cartil 27, 1800-1810

McErlain DD, Ulici V, Darling M, Gati JS, Pitelka V, Beier F, Holdsworth DW (2012): An in vivo investigation of the initiation and progression of subchondral cysts in a rodent model of secondary osteoarthritis. Arthritis Res Ther $\underline{14}$

McGibbon CA, Dupuy DE, Palmer WE, Krebs DE (1998): Cartilage and subchondral bone thickness distribution with MR imaging. Acad Radiol 5, 20-5 
Meachim G, Emery IH (1973): Cartilage fibrillation in shoulder and hip joints in Liverpool necropsies. J Anat $\underline{116}, 161-79$

Meachim G, Allibone R (1984): Topographical variation in the calcified zone of upper femoral articular cartilage. J Anat 139 ( Pt 2), 341-52

Meachim G, Whitehouse GH, Pedley RB, Nichol FE, Owen R (1980): An investigation of radiological, clinical and pathological correlations in osteoarthrosis of the hip. Clin Radiol $\underline{31}, 565-574$

Meyer EG, Baumer TG, Slade JM, Smith WE, Haut RC: Tibiofemoral contact pressures and osteochondral microtrauma during anterior cruciate ligament rupture due to excessive compressive loading and internal torque of the human knee. In: American Journal of Sports Medicine. Band 36; Am J Sports Med 2008, 1966-1977

Milgram JW (1983): Morphologic alterations of the subchondral bone in advanced degenerative arthritis. Clin Orthop Relat Res $\underline{173}$, 293-312

Miller RE, Tran PB, Obeidat AM, Raghu P, Ishihara S, Miller RJ, Malfait AM (2015): The Role of Peripheral Nociceptive Neurons in the Pathophysiology of Osteoarthritis Pain. Curr Osteoporos Rep $\underline{13}, 318-326$

Milz S., Putz R (1994): Lückenbildungen der subchondralen Mineralisierungszone des Tibiaplateaus. Osteologie $\underline{3}, 110-118$

Milz S, Putz R (1994): Quantitative morphology of the subchondral plate of the tibial plateau. J Anat $\underline{185}, 103-10$

Mital MA, Millington PF (1970): Osseous pathway of nutrition to articular cartilage of the human femoral head. Lancet 295, 842

Mummed YY (2013): Correlation between milk suckled and growth of calves of ogaden cattle at one, three and six months of age, east Ethiopia. Springerplus $\underline{2}, 302$

Muschter D, Fleischhauer L, Taheri S, Schilling AF, Clausen-Schaumann H, Grässel S (2020): Sensory neuropeptides are required for bone and cartilage homeostasis in a murine destabilization-induced osteoarthritis model. Bone 133, 115181

Nachemson A, Lewin T, Maroudas A, Freeman MAR (1970): In Vitro Diffusion of DYE Through the End-Plates and the Annulus Fibrosus of Human Lumbar Inter-Vertebral Discs. Acta Orthop Scand 41, 589-607

Nakano T, Thompson JR, Christopherson RJ, Aherne FX (1986): Blood flow distribution in hind limb bones and joint cartilage from young growing pigs. Can J Vet Res $\underline{50}$, 96-100 
Niedermair T, Kuhn V, Doranehgard F, Stange R, Wieskötter B, Beckmann J, Salmen P, Springorum HR, Straub RH, Zimmer A, et al. (2014): Absence of substance P and the sympathetic nervous system impact on bone structure and chondrocyte differentiation in an adult model of endochondral ossification. Matrix Biol 38, 22-35

Niino-Nanke Y, Akama H, Hara M, Kashiwazaki S (1998): [Alkaline phosphatase (ALP) activity in rheumatoid arthritis (RA): its clinical significance and synthesis of ALP in RA synovium]. Ryumachi $\underline{38}, 581-8$

Nwosu LN, Allen M, Wyatt L, Huebner JL, Chapman V, Walsh DA, Kraus VB (2017): Pain prediction by serum biomarkers of bone turnover in people with knee osteoarthritis: an observational study of TRAcP5b and cathepsin K in OA. Osteoarthr Cartil 25, 858-865

Occhetta P, Mainardi A, Votta E, Vallmajo-Martin Q, Ehrbar M, Martin I, Barbero A, Rasponi M (2019): Hyperphysiological compression of articular cartilage induces an osteoarthritic phenotype in a cartilage-on-a-chip model. Nat Biomed Eng $\underline{3}, 545-557$

Oegema TR, Carpenter RJ, Hofmeister F, Thompson RC (1997): The interaction of the zone of calcified cartilage and subchondral bone in osteoarthritis. Microsc Res Tech $\underline{37}$, 324-332

Oettmeier R, Abendroth K, Oettmeier S (1989): Analyses of the tidemark on human femoral heads. II. Tidemark changes in osteoarthrosis--a histological and histomorphometric study in non-decalcified preparations. Acta Morphol Hung 37, 169-80

Oftadeh R, Perez-Viloria M, Villa-Camacho JC, Vaziri A, Nazarian A (2015): Biomechanics and Mechanobiology of Trabecular Bone: A Review. J Biomech Eng 137, 0108021

Ogata K, Whiteside LA, Lesker PA (1978): Subchondral route for nutrition to articular cartilage in the rabbit. Measurement of diffusion with hydrogen gas in vivo. J Bone Joint Surg Am $\underline{60}, 905-10$

Ogino S, Sasho T, Nakagawa K, Suzuki M, Yamaguchi S, Higashi M, Takahashi K, Moriya H (2009): Detection of pain-related molecules in the subchondral bone of osteoarthritic knees. Clin Rheumatol 28, 1395-1402

Onishi K, Utturkar A, Chang E, Panush R, Hata J, Perret-Karimi D (2012): Osteoarthritis: A Critical Review. Crit Rev Phys Rehabil Med 24, 251-264

Outerbridge RE (1961): The etiology of chondromalacia patellae. J Bone Joint Surg Br $\underline{43}-\underline{B}$, $752-757$

Pan J, Zhou X, Li W, Novotny JE, Doty SB, Wang L (2009): In situ measurement of transport between subchondral bone and articular cartilage. J Orthop Res 27, 1347-1352

Pan J, Wang B, Li W, Zhou X, Scherr T, Yang Y, Price C, Wang L (2012): Elevated cross-talk between subchondral bone and cartilage in osteoarthritic joints. Bone 51, 212-217 
Pathria MN, Chung CB, Resnick DL (2016): Acute and stress-related injuries of bone and cartilage: Pertinent anatomy, basic biomechanics, and imaging perspective. Radiology $\underline{280}$, $21-38$

Pereira AF, Javaheri B, Pitsillides AA, Shefelbine SJ (2015): Predicting cortical bone adaptation to axial loading in the mouse tibia. J R Soc Interface $\underline{12}$

Perruccio A V., Power JD, Badley EM (2006): Revisiting arthritis prevalence projections - It's more than just the aging of the population. J Rheumatol $\underline{33}, 1856-1862$

Pesesse L, Sanchez C, Henrotin Y (2011): Osteochondral plate angiogenesis: A new treatment target in osteoarthritis. Jt Bone Spine $\underline{78}, 144-149$

Pham A, Hull ML (2007): Dehydration rates of meniscus and articular cartilage in vitro using a fast and accurate laser-based coordinate digitizing system. J Biomech $\underline{40}$, 3223-3229

Phinyomark A, Osis ST, Hettinga BA, Kobsar D, Ferber R (2016): Gender differences in gait kinematics for patients with knee osteoarthritis. BMC Musculoskelet Disord 17, 157

Poulet B, de Souza R, Kent A V., Saxon L, Barker O, Wilson A, Chang YM, Cake M, Pitsillides AA (2015): Intermittent applied mechanical loading induces subchondral bone thickening that may be intensified locally by contiguous articular cartilage lesions. Osteoarthr Cartil 23, 940-948

Proffen BL, McElfresh M, Fleming BC, Murray MM (2012): A comparative anatomical study of the human knee and six animal species. Knee $\underline{19}$, 493-499

Qiao M, Feld JA, Franz JR (2018): Aging effects on leg joint variability during walking with balance perturbations. Gait Posture 62, 27-33

Radin EL, Paul IL (1970): Does cartilage compliance reduce skeletal impact loads?. the relative force-attenuating properties of articular cartilage, synovial fluid, periarticular soft tissues and bone. Arthritis Rheum 13, 139-144

Radin EL, Paul IL (1971): Response of Joints to Impact Loading. I. In Vitro Wear. Arthritis Rheum $\underline{14}, 356-362$

Radin EL, Rose RM (1986): Role of subchondral bone in the initiation and progression of cartilage damage. Clin Orthop Relat Res 34-40

Radin EL, Parker HG, Pugh JW, Steinberg RS, Paul IL, Rose RM (1973): Response of joints to impact loading - III. Relationship between trabecular microfractures and cartilage degeneration. J Biomech $\underline{6}, 51-57$

Rauch A, Wohner N, Christophe OD, Denis C V., Susen S, Lenting PJ (2013): On the versatility of von Willebrand factor. Mediterr J Hematol Infect Dis $\underline{5}, 2013046$ 
Ren P, Niu H, Gong H, Zhang R, Fan Y (2018): Morphological, biochemical and mechanical properties of articular cartilage and subchondral bone in rat tibial plateau are age related. $\mathrm{J}$ Anat $\underline{232}, 457-471$

Renault JB, Carmona M, Tzioupis C, Ollivier M, Argenson JN, Parratte S, Chabrand P (2020): Tibial subchondral trabecular bone micromechanical and microarchitectural properties are affected by alignment and osteoarthritis stage. Sci Rep $\underline{10}, 1-10$

Ro DH, Lee DY, Moon G, Lee S, Seo SG, Kim SH, Park IW, Lee MC (2017): Sex differences in knee joint loading: Cross-sectional study in geriatric population. J Orthop Res $\underline{35}, 1283-$ 1289

Roemhildt ML, Coughlin KM, Peura GD, Badger GJ, Churchill D, Fleming BC, Beynnon BD (2010): Effects of increased chronic loading on articular cartilage material properties in the Lapine tibio-femoral joint. J Biomech $\underline{43}, 2301-2308$

Ryan TM, Shaw CN (2013): Trabecular bone microstructure scales allometrically in the primate humerus and femur. Proc R Soc B Biol Sci 280, 20130172-20130172

Salo PT, Seeratten RA, Erwin WM, Bray RC (2002a): Evidence for a neuropathic contribution to the development of spontaneous knee osteoarthrosis in a mouse model. Acta Orthop Scand $73,77-84$

Salo PT, Hogervorst T, Seerattan RA, Rucker D, Bray RC (2002b): Selective joint denervation promotes knee osteoarthritis in the aging rat. J Orthop Res $\underline{20}$, 1256-1264

Schenk LS: Strukturelle, quantitative und chemische Analyse der subchondralen Zone. 2010

Schilling AF: Subchondral Bone in Articular Cartilage Regeneration. In: Cartilage. Springer International Publishing, Cham 2017, 139-150

Schindelin J, Arganda-Carreras I, Frise E, Kaynig V, Longair M, Pietzsch T, Preibisch S, Rueden C, Saalfeld S, Schmid B, et al. (2012): Fiji: an open-source platform for biologicalimage analysis. Nat Methods $\underline{9}, 676-682$

Schultz M, Molligan J, Schon L, Zhang Z (2015): Pathology of the calcified zone of articular cartilage in post-traumatic osteoarthritis in rat knees. PLoS One 10, 120949

Schumacher HR: Crystal-induced arthritis: An overview. In: American Journal of Medicine. Band 100; Am J Med 1996

Schwab W, Bilgiçyildirim A, Funk RHW (1997): Microtopography of the autonomic nerves in the rat knee: A fluorescence microscopic study. Anat Rec 247, 109-118

Seo S-S, Kim C-W, Jung D-W (2011): Management of Focal Chondral Lesion in the Knee Joint. Knee Surg Relat Res 23, 185-196 
Shah MR, Kaplan KM, Meislin RJ, Bosco JAArticular Cartilage Restoration of the Knee. 2007

Shapiro R, Heaney RP (2003): Co-dependence of calcium and phosphorus for growth and bone development under conditions of varying deficiency. Bone $32,532-540$

Slattery C, Kweon CY (2018): Classifications in Brief: Outerbridge Classification of Chondral Lesions. Clin Orthop Relat Res $\underline{476}, 2101-2104$

Smith LK, Lelas JL, Kerrigan DC (2002): Gender Differences in Pelvic Motions and Center of Mass Displacement during Walking: Stereotypes Quantified. J Womens Health Gend Based Med 11, 453-458

Sniekers YH, Intema F, Lafeber FPJG, Van Osch GJVM, Van Leeuwen JPTM, Weinans H, Mastbergen SC (2008): A role for subchondral bone changes in the process of osteoarthritis; A micro-CT study of two canine models. BMC Musculoskelet Disord $\underline{9}, 20$

Starke RD, Ferraro F, Paschalaki KE, Dryden NH, McKinnon TAJ, Sutton RE, Payne EM, Haskard DO, Hughes AD, Cutler DF, et al. (2011): Endothelial von Willebrand factor regulates angiogenesis. Blood $\underline{117}, 1071-1080$

Stavenschi E, Corrigan MA, Johnson GP, Riffault M, Hoey DA (2018): Physiological cyclic hydrostatic pressure induces osteogenic lineage commitment of human bone marrow stem cells: A systematic study. Stem Cell Res Ther 9

Steadman JR, Rodkey WG, Rodrigo JJ: Microfracture: Surgical technique and rehabilitation to treat chondral defects. In: Clinical Orthopaedics and Related Research. Lippincott Williams and Wilkins 2001

Steadman JR, Rodkey WG, Briggs KK (2002): Microfracture to treat full-thickness chondral defects: surgical technique, rehabilitation, and outcomes. J Knee Surg $\underline{15}$, 170-6

Stewart HL, Kawcak CE (2018): The importance of subchondral bone in the pathophysiology of osteoarthritis. Front Vet Sci $\underline{5}, 178$

Stockwell RA, Barnett CH (1964): Changes in Permeability of Articular Cartilage with Age. Nature 201, 835-836

Suri S, Walsh DA (2012): Osteochondral alterations in osteoarthritis. Bone 51, 204-211

Suri S, Gill SE, De Camin SM, Wilson D, McWilliams DF, Walsh DA (2007): Neurovascular invasion at the osteochondral junction and in osteophytes in osteoarthritis. Ann Rheum Dis $\underline{66}, 1423-1428$

Taheri S, Winkler T, Schenk L, Neuerburg C, Baumbach S, Zustin J, Lehmann W, Schilling A (2019): Developmental Transformation and Reduction of Connective Cavities within the Subchondral Bone. Int J Mol Sci $\underline{20}, 770$ 
Teshima R, Nawata K, Hagino H, Morio Y, Inoue M, Irizawa Y (1999): Effects of weight bearing on the tidemark and osteochondral junction of articular cartilage: Histomorphometric analyses of 7 normal femoral heads. Acta Orthop Scand $\underline{70}$, 381-386

Thambyah A, Broom N On how degeneration influences load-bearing in the cartilageebone system: a microstructural and micromechanical study.

Turmezei TD, Lomas DJ, Hopper MA, Poole KES (2014): Severity mapping of the proximal femur: A new method for assessing hip osteoarthritis with computed tomography. Osteoarthr Cartil 22, 1488-1498

Tuzmen C, Campbell PG (2018): Crosstalk between neuropeptides SP and CGRP in regulation of BMP2-induced bone differentiation. Connect Tissue Res 59, 81-90

Tuzmen C, Verdelis K, Weiss L, Campbell P (2018): Crosstalk between substance P and calcitonin gene-related peptide during heterotopic ossification in murine Achilles tendon. $\mathrm{J}$ Orthop Res $\underline{36}, 1444-1455$

Van Spil WE, Kubassova O, Boesen M, Bay-Jensen AC, Mobasheri A (2019): Osteoarthritis phenotypes and novel therapeutic targets. Biochem Pharmacol 165, 41-48

Verborgt O, Gibson GJ, Schaffler MB (2000): Loss of osteocyte integrity in association with microdamage and bone remodeling after fatigue in vivo. J Bone Miner Res $\underline{15}, 60-67$

Vorwerg Y, Petroff D, Kiess W, Blüher S (2013): Physical Activity in 3-6 Year Old Children Measured by SenseWear Pro®: Direct Accelerometry in the Course of the Week and Relation to Weight Status, Media Consumption, and Socioeconomic Factors. PLoS One $\underline{8}$, e60619

Wang Y, Wluka AE, Pelletier JP, Martel-Pelletier J, Abram F, Ding C, Cicuttini FM (2010): Meniscal extrusion predicts increases in subchondral bone marrow lesions and bone cysts and expansion of subchondral bone in osteoarthritic knees. Rheumatology (Oxford) $\underline{49}$, 997-1004

Wang Y, Wei L, Zeng L, He D, Wei X (2013): Nutrition and degeneration of articular cartilage. Knee Surg Sports Traumatol Arthrosc 21, 1751-62

Wilsman NJ, Van Sickle DC (1972): Cartilage canals, their morphology and distribution. Anat $\operatorname{Rec} \underline{173}, 79-93$

Winkler T: Die osteochondrale Zone strukturelle und zelluläre Untersuchung. Shaker 2012 Woods CG, Greenwald AS, Haynes DW (1970): Subchondral vascularity in the human femoral head. Ann Rheum Dis 29, 138-142 
Yan J-Y, Tian F-M, Wang W-Y, Cheng Y, Xu H-F, Song H-P, Zhang Y-Z, Zhang L (2014): Age Dependent Changes in Cartilage Matrix, Subchondral Bone Mass, and Estradiol Levels in Blood Serum, in Naturally Occurring Osteoarthritis in Guinea Pigs. Int J Mol Sci $15,13578-13595$

Yuan XL, Meng HY, Wang YC, Peng J, Guo QY, Wang AY, Lu SB (2014): Bone-cartilage interface crosstalk in osteoarthritis: potential pathways and future therapeutic strategies. Osteoarthr Cartil 22, 1077-1089

Zhen G, Wen C, Jia X, Li Y, Crane JL, Mears SC, Askin FB, Frassica FJ, Chang W, Yao J, et al. (2013): Inhibition of TGF- $\beta$ signaling in mesenchymal stem cells of subchondral bone attenuates osteoarthritis. Nat Med $\underline{19}, 704-712$

Zimmer A, Zimmer AM, Baffi J, Usdin T, Reynolds K, König M, Palkovits M, Mezey E (1998): Hypoalgesia in mice with a targeted deletion of the tachykinin 1 gene. Proc Natl Acad Sci U S A $\underline{95}, 2630-2635$ 


\section{Acknowledgement}

AG Schilling

I would like to thank all the scientists and technicians of the interdisciplinary research group of Prof. Arndt Schilling. First, I would like to mention Ramona Castro-Machguth, Kathrin Hannke, Andrea Koch, and Annette Witt for their excellent technical assistance, as well their patience with me to improve my German comprehension. Special thanks to Dr. Kai Böker for being an ever-present source of helpful discussions, for his general positivity, and his support for the PCR-based sex determination of the femoral heads. I also like to thank Dr. Takashi Yoshida and Robert H. Förster for conducting the gait analysis experiment at the Applied Surgical and Rehabilitation Technology Lab (ARTLab). Many thanks to Lina Jochim who did her MD thesis with me, and was a massive help in the lab for scanning the healthy femoral heads. Finally, I would like to thank Prof. Schilling who acquired the financial support for my thesis through the German Research Foundation (DFG) under the subproject 5 [SCHI 857/9-1] of the Research Consortium ExCarBon /4 FOR2407/1. I am extremely grateful for his trust in me, for his seemingly incessant enthusiasm for science, and for providing an ideal balance between supervised and independent research.

Prof. Alves, PD Dr. sc. hum. Dullin, and Prof. Wichmann

I would like to express my deepest appreciation to my thesis committee. Specifically, Prof. Alves for her great support during my $\mathrm{PhD}$, and for organizing the $2^{\text {nd }}$ molecular imaging symposium at the Max-Planck-Institute for Experimental Medicine that was a great source of fruitful discussions. I would like to express my sincere gratitude to Dr. Christian Dullin to share his valuable expertise with me, and for helping to acquire beamtimes at the Canadian Light Source synchrotron facility. Additionally, I'd also like to extend my gratitude to Prof. Wichmann who accepted to be part of my thesis committee, for the nice atmosphere during my committee meetings and all the tips and suggestions provided there. 
Prof. Kornak. and Prof. Siggelkow

I would like to express my great appreciation to Prof. Kornak and Prof. Siggelkow who accepted to be on the examination committee. Their valuable and constructive suggestions, as well as their willingness to allocate their time so generously is very much appreciated.

Technische Universität Hamburg-Harburg \& Universitätsklinikum Hamburg-Eppendorf I would like to thank Dr. Thomas Winkler and Lia Sabrina Schenk at TUHH for acquiring the bovine biopsies and sharing their expertise in micro-CT imaging. Furthermore, many thanks to Dr. Jozef Zustin for developing and conducting the Mason-Goldner-based histomorphometrical methods for the bovine bone.

Medizinische Hochschule Hannover \& Department of Trauma Surgery, Orthopedics and Plastic Surgery

I would also like to thank the anonymous body donors and the $M H H$ 's Institute for Functional and Applied Anatomy for their donation of the bone samples, and Andreas Buchhorn for his support with the samples. Likewise, I would like to thank the CUOP orthopaedic surgeons, Prof. Frank Timo Beil, Dr. Jan Hubert, Dr. Thelonius Hawellek, and Prof. Wolfgang Lehmann for classification of the bones according to the Outerbridge system.

Johann-Friedrich-Blumenbach-Institute for Zoology \& Anthropology, Göttingen An enormous amount of gratitude goes to Anna-Lena Flux and Dr. Birgit Grosskopf for the biological age determination of the femoral heads.

\section{AG Grässel}

The research group "Experimental Orthopaedics, Centre for Medical Biotechnology" of the University of Regensburg headed by Prof. Susanne Grässel was a major cooperation partner in the ExCarBon consortium, and therefore, receives my sincere gratitude. Special thanks to Dr. Dominique Muschter for providing the WT, and sensory neuropeptide- 
deficient mice samples. Also, I would like to thank Anja Pasoldt and Tanja Späth for their outstanding technical assistance in Safranin-O histology stainings. 